Thomas Kuhn

\title{
Schlüsselzuweisungen und fiskalische Ungleichheit
}

Eine theoretische Analyse der Verteilung von Schlüsselzuweisungen an Kommunen 


\section{Thomas Kuhn}

\section{Schlüsselzuweisungen und fiskalische Ungleichheit}

Die Schlüsselzuweisungen haben im kommunalen Finanzausgleich im wesentlichen die Aufgabe, die fiskalische Ungleichheit der Kommunen zu reduzieren. Ob dies auch wirklich gelingt, ist mit analytischen Methoden noch nicht untersucht worden. Das liegt in erster Linie an dem numerischen Iterationsverfahren, mit dem die Schlüsselzuweisungen berechnet werden, und der Intransparenz, die daraus resultiert. Die vorliegende Arbeit erklärt nun erstmals analytisch, von welchen Einflußgrößen die Verteilung der Schlüsselzuweisungen abhängt und wie sie sich auf die fiskalische Ungleichheit der Kommunen auswirkt.

Thomas Kuhn, Jahrgang 1955, studierte Wirtschaftsingenieurwesen an der Universität Karlsruhe. Diplom 1981. Danach wiss. Mitarbeiter am Lehrstuhl für VWL V der Universität Augsburg Promotion 1985. Seitdem akad. Rat a.Z. am gleichen Lehrstuhl. 
Schlüsselzuweisungen und fiskalische Ungleichheit

Eine theoretische Analyse der Verteilung von

Schlüsselzuweisungen an Kommunen 


\section{FINANZWISSENSCHAFTLICHE SCHRIFTEN}

Herausgegeben von den Professoren

Albers, Krause-Junk, Littmann, Oberhauser, Pohmer, Schmidt

Band 36

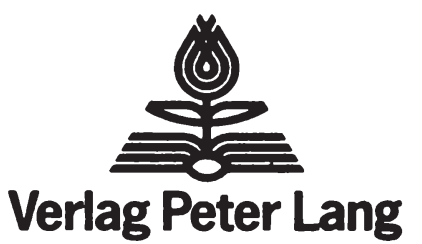

Frankfurt am Main · Bern · New York · Paris 


\section{Thomas Kuhn}

\section{Schlüsselzuweisungen und fiskalische Ungleich heit}

Eine theoretische Analyse der Verteilung von Schlüsselzuweisungen an Kommunen

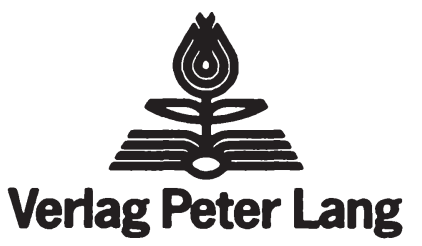

Frankfurt am Main - Bern - New York - Paris 
CIP-Kurztitelaufnahme der Deutschen Bibliothek

Kuhn, Thomas:

Schlüsselzuweisungen und fiskalische Ungleichheit : $\theta$. theoret. Analyse d. Verteilung von Schlüsselzuweisungen an Kommunen / Thomas Kuhn. - Frankfurt am Main ; Bern ; New York ;

Paris : Lang, 1988

(Finanzwissenschaftliche Schriften ; Bd. 36)

Zugl.: Augsburg, Univ., Diss., 1985

ISBN 3-8204-0051-6

NE: GT

Open Access: The online version of this publication is published on www.peterlang.com and www.econstor.eu under the international Creative Commons License CC-BY 4.0. Learn more on how you can use and share this work: http://creativecommons. org/licenses/by/4.0.

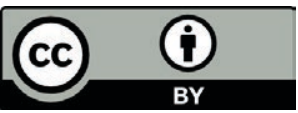

This book is available Open Access thanks to the kind support of ZBW - Leibniz-Informationszentrum Wirtschaft.

\section{384}

ISSN 0170-8252

ISBN 3-8204-0051-6

ISBN 978-3-631-75193-0 (eBook)

c) Verlag Peter Lang GmbH, Frankfurt am Main 1988 Alle Rechte vorbehalten.

Das Werk einschlieBlich aller seiner Teile ist urheberrechtlich geschützt. Jede Verwertung außerhalb der engen Grenzen des Urheberrechtsgesetzes ist ohne Zustimmung des Verlages unzulässig und strafbar. Das gilt insbesondere für Vervielfältigungen, Übersetzungen, Mikroverfilmungen und die Einspeicherung und Verarbeitung in elektronischen Systemen.

Druck und Bindung: Weihert-Druck GmbH, Darmstadt

Printed in Germany 


\section{INHALTSVERZEICHNIS}

Seite

Einführung

I KOMMUNALER FINANZAUSGLEICH, SCHLUSSELZUWE I SUNGEN UND VERTEILUNGSZIELE

1 Der kommunale Finanzausgleich 9

1.1 Begriffsbestimmungen 9

1.2 Zuweisungsarten 11

1.3 Finanzierung der Zuweisungen 12

2 Das System der Schlüsselzuweisungen $\quad 15$

2.1 Die Schlüsselzuweisungen $\quad 15$

2.1.1 Der Ausgleich zwischen Finanzbedarf $\begin{array}{ll}\text { und Steuerkraft } & 15\end{array}$

2.1.2 Die Ausschöpfung der Schlüsselmasse $\begin{array}{ll}\text { und das Versorgungsniveau } & 24\end{array}$

2. 2 Sonderschlüsselzuweisungen und Mindest-
garantien

2.2.1 Sonderschlüsselzuweisungen $\quad 32$

2.2.2 Mindestgarantien 38

2.3 Existenz und Eindeutigkeit der Schlüssel- 46

2.4 Iterationsverfahren für die Berechnung
des Grundbetrags

2.4.1 Verfahren (1) 49

2.4.2 Das Verfahren

2.4.3 Ein modifiziertes Verfahren (3) 55

3 Verteilungsziele und Verteilungskriterien 59

3.1 Kommunale ziele 59

$\begin{array}{lll}3.2 & \text { Staatliche Ziele } & 64\end{array}$

3.3 Kriterien der fiskalischen Ungleichheit 71 
4 Die fiskalische Ungleichheit der Gemeinden

4.1 Finanzierungsdefizite und Finanzierungsüberschüsse in den Gemeinden

4.2 Die Nichtregressivität der Schlüsselzuweisungen

4.3 Bedingungen für fiskalische Gleichheit

5 Verteilungen bei variabler Schlüsselmasse

5.1 Die Abhängigkeit der Schlüsselzuweisungen von der Schlüsselmasse

5.1.1 Der Grundbetrag als Funktion der Schlüsselmasse

Exkurs: Verfahren (4) für die Berechnung des Grundbetrags

100

5.1.2 Die Schlüsselzuweisungen als Funktion

der Schlüsselmasse

101

5.2 Optimale Schlüsselmassen für Kommunen

104

5.2.1 Die Maximierung der Finanzausstattung 104

5.2.2 Die Maximierung des Finanzanteils 105

5.3 Die Variation der Schlüsselmasse aus Sicht von Gemeindegruppen

5.3.1 Die Verteilung der Finanzausstattungen 114

5.3.2 Die Umverteilung der Finanzanteile 120

5.4 Progressive und regressive Verteilungen 129

6 Verteilungen bei variabler Ausschüttungsquote 134

6.1 Die Schlüsselzuweisungen als Funktion der Ausschüttungsquote

6.2 Optimale Ausschüttungsquoten für Kommunen 144

6.3 Die Variation der Ausschüttungsquote aus der Sicht von Gemeindegruppen

6.3.1 Die Umverteilung der Finanzausstattungen und Finanzanteile 
Seite

6.3.2 Verringerung der Ausschüttungsquote 158

6.3.3 Erhöhung der Ausschüttungsquote 165

$\begin{array}{lll}6.4 & \text { Progressive und regressive Vertellungen } & 167\end{array}$

7 Analyse der Sonderschlüsselzuweisungen und
Mindestgarantien

I I KRITIK UND REFORMVORSCHLÄGE

8 Grenzen der Redistribution $\quad 179$

9 Ein alternatives System von Zuweisungen 186

$\begin{array}{ll}\text { SchluBbemerkungen } & 190\end{array}$

$\begin{array}{ll}\text { Zusammenfassung } & 193\end{array}$

Verzeichnis der wichtigsten Symbole 202

Verzeichnis der Gesetze 204

Literaturverzeichnis 206 
Thomas Kuhn - 978-3-631-75193-0

Downloaded from PubFactory at 01/11/2019 07:16:43AM

via free access 


\section{Einfürung}

Die Schlüsselzuweisungen können zweifellos als das Kernstück des kommunalen Finanzausgleichs gelten. Es dürfte allgemein bekannt sein, daß sie einen großen Teil der gesamten Zuweisungen eines Landes an die Kommunen ausmachen. Im Jahre 1984 etwa waren es im Durchschnitt der Bundesländer ca. 418. 1) Doch die Schlüsselzuweisungen haben nicht nur wegen ihres Volumens eine große Bedeutung, sondern vor allem wegen der Art und Weise, wie sie vergeben werden. Wie der Name schon sagt, sind dies Zuweisungen, die nach einem regelgebundenen Verfahren, eben einem Schlüssel, auf die Kommunen verteilt werden. Danach erhält eine Kommune einen bestimmten Prozentsatz des Unterschieds zwischen ihrem Finanzbedarf und ihrer Steuerkraft als Schlüsselzuweisung. Diesen Prozentsatz bezeichnet man als Ausschüttungsquote. Kommunen, deren Steuerkraft mindestens so hoch wie der Finanzbedarf ist, sind nicht zuweisungsberechtigt.

Diese Grundstruktur geht letztlich auf J. Popitz zurück und findet sich schon im Preußischen Finanzausgleichsgesetz von 1938. 2) Auch die Ziele, die man mit Schlüsselzuweisungen verfolgt, sind dort schon zutreffend formuliert und in ihrem Kern bis in die heutige zeit hinein gültig geblieben: "Durch diese zuweisungen sollen bei den einzelnen Gemeinden die Unterschiede gemildert werden, die zwischen ihrer eigenen Steuerkraft und ihrer Ausgabenbelastung bestehen. Dabei sollen ... diejenigen Gemeinden besser gestellt werden, deren eigene Einnahmemöglichkeiten sich als nicht ausreichend erwiesen haben." 3) Etwas präziser könnte man hier auch vom

1) Siehe Karrenberg, Münstermann [1985], S. 89.

2) Siehe Popitz [1932] u. Preußisches Finanzausgleichsgesetz vom 10. Nov. 1938. Zur Entstehungsgeschichte der Schlüsselzuweisungen siehe Hansmeyer, Kops [1985].

3) Preußisches Finanzausgleichsgesetz vom 10. Nov. 1938, §11. 
Fiskal- und Verteilungsziel sprechen, wenn man darunter zum einen eine Verbesserung der kommunalen Finanzausstattung und zum anderen eine Reduzierung der fiskalischen Ungleichheit unter den Kommunen versteht.

Ob die Schlüsselzuweisungen diesen Forderungen überhaupt genügen, ist von vornherein keineswegs gesagt, denn das ganze system erweist sich letztlich als relativ komplex. Das liegt einfach daran, daß in jedem Jahr immer nur eine fest vorgegebene summe, die sogenannte Schlüsselmasse, auf die Kommunen verteilt werden kann. Sie wird im Grunde von den Steuereinnahmen eines Landes begrenzt. Um die verfügbare Schlüsselmasse genau auszuschöpfen, muß im System der Schlüsselzuweisungen der Finanzbedarf der Kommunen endogenisiert werden.

Dieser setzt sich multiplikativ aus der veredelten Einwohnerzahl, die als eine gemeindespezifische Bedarfskennziffer anzusehen ist, und dem sogenannten Grundbetrag zusammen. Der Grundbetrag ist für alle Gemeinden gleich und wird endogen so bestimmt, daß sich ein Gleichgewicht zwischen der Schlüsselmasse und der summe der zuweisungen ergibt. Man kann ihn daher auch als durchschnittliches Versorgungsniveau interpretieren. Ordnet man alle Gemeinden nach ihrer relativen Armut, dann gibt es $z$ u einer bestimmten Schlüsselmasse und dem dazugehörigen Versorgungsniveau eine ganz bestimmte Gemeinde in dieser ordnung, die gerade noch $\mathrm{Zu}-$ weisungen erhält. Alle reicheren Gemeinden sind nicht mehr zuweisungsberechtigt, man sagt auch, sie seien abundant. Für die relative Armut einer Kommune ist dabei das Verhältnis aus ihrer steuerkraft und ihrer Bedarfskennziffer maBgebend.

Man kann sich nun leicht vorstellen, daß sich mit dem Versorgungsniveau auch die $\mathrm{zahl}$ der zuweisungsberechtigten 
Gemeinden ändert. Insofern ist bei der Berechnung des Versorgungsniveaus eine gewisse Interdependenz $z u$ beachten, die sich in einer nur implizit bestimmten Gleichgewichtslösung ausdrückt. Vereinfacht muß man sich das so vorstellen: Einerseits hängt es vom Versorgungsniveau $a b$, ob eine Gemeinde Zuweisungen erhält, andererseits ist das Versorgungsniveau wiederum davon abhängig, welche Gemeinden $z u-$ weisungsberechtigt sind. Die Statistischen Landesämter behelfen sich bei diesem Problem mit einem numerischen Iterationsverfahren. In jedem Schritt wird dabei eine Annahme über die zuweisungsberechtigten Gemeinden getroffen, mit dieser Annahme ein Versorgungsniveau ermittelt und dann überprüft, ob die Gemeinden, die bei diesem Versorgungsniveau zuweisungsberechtigt wären, mit denen aus der Annahme identisch sind. Dieses procedere wiederholt sich so lange, bis sich zur Annahme kein Widerspruch mehr ergibt.

Das ganze System wird dadurch natürlich sehr unhandich, ja, man könnte sogar behaupten, "daß die jetzige Regelung kaum noch zu durchschauen ist". "Jedenfalls fällt es außerordentlich schwer, die Verteilungsergebnisse der Schlüsselzuweisungen theoretisch zu erklären, d.h. auf den EinfluB einzelnex Größen im System zurückzuführen. ${ }^{2)}$ Dies kann m. E. nur eine mathematische Analyse mit der notwendigen Allgemeingültigkeit leisten. Umso mehr muß es verwundern, daß noch keine Untersuchung bekannt geworden ist, die sich einer fomalen Methodik bedienen würde. Immerhin ist das System in seinen Grundzügen nun schon seit annähernd 50 Jahren in die Finanzpraxis eingeführt und seine Intransparenz wird in der Literatur immer wieder beklagt. ${ }^{3)}$
1) Vgl. Deubel [1985], S. 76.
2) Vgl. Postlep [1985], s. 202.
3) Vgl. zuletzt Postlep [1985], S. 204. 
Als Einflußgrößen sind die Schlüsselmasse und die Ausschüttungsquote von Interesse, insbesondere auch unter dem Aspekt, sie für die Verteilungsziele des Staates $z u$ instrumentalisieren. Um die Verteilungsergebnisse mit ihnen erklären zu können, wird man sie, rein technisch gesehen, als unabhängige Variablen behandeln. Die übrigen Größen nimmt man als exogen gegeben an. Es kommt dann darauf an, die Schlüsselzuweisungen und die dazugehörige Verteilung von den Variablen funktional abhängig zu machen. Dann kann man auf theoretischem Wege beliebig viele alternative Verteilungen erzeugen und sie hinsichtlich der Verteilungsziele miteinander vergleichen. Eine notwendige Voraussetzung dafür ist jedoch, das Versorgungsniveau als diejenige endogene Variable, die das system bei jeder Verteilung ins Gleichgewicht bringt, explizit als Funktion der Variablen $z u$ bestimmen. Ein numerisches Iterationsverfahren ist für die theoretische Arbeit natürlich unbrauchbar, denn damit läßt sich mit einigem Aufwand immer nur eine ganz bestimmte Verteilung empirisch berechnen, wenn man die exogenen Größen und die unabhängigen Variablen spezifiziert.

In der Literatur ist es noch nicht gelungen, das Versorgungsniveau analytisch zu bestimmen. Dort findet sich zwar eine Gleichung, die meist als explizite Darstellung des Versorgungsniveaus interpretiert wird und dann für weitreichende Behauptungen uber die Verteilungswirkungen einzelner Variablen herhalten muß. Dafür ist sie aber überhaupt nicht geeignet, weil sie die $\mathrm{Zahl}$ der zuweisungsberechtigten Gemeinden als bekannt voraussetzt und von den vorhandenen Interdependenzen in unzulässiger Weise abstrahiert. Vom mathematischen standpunkt aus gesehen taugt sie in dieser vereinfachten Form noch nicht einmal 
für eine implizite Formulierung des Gleichgewichts. ${ }^{1)}$ Nun wird man auch verstehen, weshalb die Schlüsselzuweisungen mit formalen Methoden noch nicht untersucht worden sind.

Angesichts des bestehenden Forschungsdefizits hat sich diese Arbeit zum ziel gesetzt, die Verteilungsergebnisse der Schlüsselzuweisungen theoretisch zu erklären. Dazu müssen wir uns, genau wie wir es oben skizziert haben, beliebig viele alternative Verteilungen beschaffen. Wenn man staatliche und kommunale ziele präzisiert, dann kann man diese Verteilungen auf ihre Eigenschaften hin überprüfen. Der staat ist an Verteilungen interessiert, mit denen die fiskalische Ungleichheit der Kommunen reduziert wird. Darüberhinaus ist aus staatlicher sicht auch zu fragen, ob es überhaupt eine Verteilung gibt, mit der slch fiskalische Gleichheit realisieren ließe. Eine Kommune wird eine Verteilung in erster Linie danach beurteilen, ob sie mit den Schlüsselzuweisungen, die sie bei dieser Verteilung erhält, ihre eigene Position absolut und relativ verbessern kann.

Zur Behandlung dieser Fragen bedienen wir uns durchweg eines formalen Instrumentariums, das sich allerdings, wie man sich leicht vorstellen kann, teilweise als recht unhandlich erweist. Das liegt einfach an der Komplexität des Systems, die sich zwangsläufig in sehr "unschönen" Funktionen für das Versorgungsniveau und alle davon abgeleiteten Größen zeigt. Der formale Aufwand rechtfertigt sich jedoch wieder durch teilweise überraschende Resultate. Einen anderen Teil der Ergebnisse konnte man vielleicht intuitiv so erwarten, eine richtige Intuition kann

1) Zum Beweis dieser These siehe ausführlich Abschnitt 2.1.2 und die dort angegebene Literatur 
aber natürlich keinen strengen Beweis ersetzen. Im einzelnen gehen wir in der Untersuchung wie folgt vor.

Nach einer kurzen Einordnung der Schlüsselzuweisungen in den kommunalen Finanzausgleich wird im 2. Kapitel zunächst das System der Schlüsselzuweisungen in den einzelnen Bundesländern dargestellt, auf eine gemeinsame Grundstruktur reduziert und formalisiert. Anschließend gehen wir ausführlich auf das Problem der analytischen Bestimmung des Versorgungsniveaus ein, für das es in der Literatur bisher noch keine Lösung gab. In diesem zusammenhang ist auch zu zeigen, daß ein gleichgewichtiges Versorgungsniveau existiert und daß es eindeutig ist. Dieser Nachweis ist zudem eine notwendige Voraussetzung für die Anwendung von Iterationsverfahren. $\mathrm{Zu}$ dem in den Statistischen Landesämtern praktizierten Verfahren geben wir eine genaue algorithmische Beschreibung sowie drei weitere Alternativen an, die sogar noch etwas einfacher und schneller sein dürften.

Im 3. Kapitel kommt es im wesentlichen darauf an, kommunale und staatliche ziele zu präzisieren und daraus Kriterien für die Beurteilung von Verteilungen zu entwickeln. Aus staatlicher sicht wird von einer Verteilung insbesondere die Progressivität gefordert. Hier ist es allerdings erst mit einer geeigneten Umformulierung des Lorenzkurven-Kriteriums möglich, die Progressivität einer Verteilung auf analytischem Wege einzuschätzen. Aus der Sicht einer Kommune ist eine Verteilung optimal, mit der die Finanzausstattung bzw. der Finanzanteil der Kommune maximiert wird.

Im zweiten Teil wird das system der Schlüsselzuweisungen unter den genannten Gesichtspunkten analysiert und beur- 
teilt. Zunächst vergleichen wir im 4. Kapitel die Situation bei einer beliebigen Verteilung der Schlüsselzuweisungen mit einer situation, in der die Kommunen nur über ihre eigenen Steuereinnahmen verfügen können. Dazu betrachten wir Finanzierungsüberschüsse und Finanzierungsdefizite, die in den Gemeinden trotz $\mathrm{z}$ uweisungen noch verbleiben und geben Bedingungen an, unter denen die fiskalische Ungleichheit mit Zuweisungen völlig zum Verschwinden gebracht werden kann.

Im 5. Kapitel vergleichen wir Verteilungen miteinander, die durch eine Variation der Schlüsselmasse erzeugt werden. Die Grundlage dafür ist ein funktionaler Zusammenhang zwischen dem Versorgungsniveau und der schlüsselmasse, mit dem man die zuweisungen an die Kommunen und die dazugehörigen Verteilungen von der Schlüsselmasse funktional abhängig machen kann. Für die einzelnen Kommunen werden dann optimale Schlüsselmassen bestimmt. Danach untersuchen wir, wie sich eine Erhöhung oder Verringerung der Schlüsselmasse um einen bestimmten Betrag auf Gemeinden und insbesondere Gemeindegruppen auswirkt, wenn man von der Schlüsselmasse im status quo ausgeht. Mit dem Nachweis, daß Verteilungen mit höherer Schlüsselmasse progressiver sind als Verteilungen mit niedrigerer Schlüsselmasse, schließt das Kapitel ab.

Das 6. Kapitel geht prinzipiell den gleichen Fragen wie das vorherige nach, nur mit dem Unterschied, daß die alternativen Verteilungen diesmal nicht aus einer Variation der Schlüsselmasse, sondern der Ausschüttungsquote resultieren. Ganz analog zum 5. Kapitel geben wir das Versorgungsniveau, die Schlüsselzuweisungen und die dazugehörige Verteilung als Funktion der Ausschütungsquote an. Man kann dann wieder optimale Ausschüttungsquoten für die Kommunen, die Umverteilung zwischen Gemeindegruppen bei einer Änderung 
des status quo und die Regressivität bzw. Progressivität von Verteilungen bestimmen.

Im 7. Kapitel beziehen wir Sonderschlüsselzuweisungen und Mindestgarantien in die Analyse ein. Ihre Funktionsweise ist zuvor schon im 2. Kapitel ausführlich erläutert worden. Ihre Verteilungswirkungen werden nun an einer situation gemessen, in der man die für Sonderschlüsselzuweisungen und Mindestgarantien aufgewendeten Finanzmittel alternativ nur für Schlüsselzuweisungen einsetzen würde.

Im 8. Kapitel zeigen wir systemimmanente Grenzen der Redistribution auf, wobei wir uns auch auf die im II. Teil gewonnenen Erkenntnisse stützen. Aus dieser Kritik leitet sich im 9. Kapitel quasi zwangsläufig ein alternatives, viel handlicheres system von zuweisungen $a b$, mit dem sich insbesondere das Ziel der fiskalischen Gleichheit sehr einfach erreichen läßt.

Mit einer Zusammenfassung der wichtigsten Erkenntnisse, einigen Anmerkungen $z u$ ihrer praktischen Anwendung und einem Ausblick auf die weitere Forschung schließt die Arbeit ab. 
I KOMMUNALER FINANZAUSGLEICH, SCHLÜSSELZUWEISUNGEN UND VERTEILUNGSZIELE

1 Der kommunale Finanzausgleich

In der Bundesrepublik Deutschland muB, wie in jedem föderalistisch aufgebauten staat, über die Verteilung der öffentlichen Aufgaben auf die Gebietskörperschaften, das sind Bund, Länder und Gemeinden, entschieden werden. Gibt man die Aufgabenverteilung vor, dann ist damit natürlich auch eine bestimmte Verteilung der Ausgaben impliziert. Durch eine entsprechende Verteilung der Staatseinnahmen ist dann dafür zu sorgen, daß die einzelnen Gebietskörperschaften ihre Ausgaben auch finanzieren können. Dazu werden fast immer Zuweisungen zwischen Gebietskörperschaften notwendig, denn es kommt praktisch nie vor, daß die Einnahmen einer Körperschaft genau ihrem Ausgabenbedarf entsprechen.

\subsection{Begriffsbestimmungen}

Nach diesen kurzen Vorbemerkungen können wir nun definieren, was man unter Finanzausgleich versteht. Finanzausgleich bedeutet die Zuordnung der Aufgaben, Ausgaben und Einnahmen auf Gebietskörperschaften. ${ }^{1)}$ Im kommunalen Finanzausgleich werden also Aufgaben, Ausgaben und Einnahmen auf Kommunen auf der einen, und auf Bund und Land auf der anderen Seite verteilt, bzw. auf Kommunen und Land, wenn man von den spärlichen Beziehungen zwischen Bund und Kommunen abstrahiert. Nimmt man die Ausgaben- und Einnahmenverteilung als gegeben an, dann reduziert sich der kommunale Finanzausgleich nur noch auf die Zuweisungen zwischen Land und Kommunen. Zuweisungen sind dabei.allgemein definiert als Zahlungen zwischen

1) Siehe Zimmermann, Henke [1982], S. 89 . Von sonstigen öffentlichen Körperschaften, wie Sozialversicherungen, wird abstrahiert. 
Körperschaften. Man benötigt sie einfach deshalb, weil die eigenen Einnahmen einer Körperschaft praktisch nie ihrem Ausgabenbedarf entsprechen.

Zuweisungen gibt es nicht nur zwischen über- und nachgeordneten Körperschaften, sondern auch zwischen Körperschaften der gleichen Ebene. Im einen Fall spricht man vom vertikalen, im anderen Fall vom horizontalen Finanzausgleich. In der Praxis jedoch lassen sich horizontaler und vertikaler Finanzausgleich nicht ganz voneinander trennen. Dies trifft insbesondere auf den kommunalen Finanzausgleich $\mathrm{zu}$, den man als vertikalen Finanzausgleich mit horizontalem Effekt charakterisieren könnte. Was ist darunter $\mathrm{zu}$ verstehen?

Zwischen den Kommunen bestehen normalerweise erhebliche fiskalische Unterschiede. So gibt es relativ arme Kommunen, deren Einnahmen zur Deckung ihres Finanzbedarfs nicht ausreichen und relativ reiche, die sogar einen Uberschuß ihrer Einnahmen über ihren Finanzbedarf aufweisen. Diese fiskalische Ungleichheit unter den Gemeinden soll mit dem horizontalen Finanzausgleich reduziert werden. Dieses $\mathrm{Ziel}$ könnte man auch leicht mit Umlagen erreichen, also mit Zahlungen der reicheren Kommunen an die ärmeren.

Im kommunalen Finanzausgleich wird jedoch meist so vorgegangen, daß ein Land für zuweisungen an Kommunen eine bestimmte Finanzmasse zur Verfügung stellt, mit der die Kommunen an den steuereinnahmen des Landes beteiligt werden (steuerverbund, siehe 1.3). Damit läßt sich natürlich die Finanzausstattung der Kommunen ganz allgemein erhöhen. Dies ist jedoch nicht das primäre ziel und auch nicht weiter problematisch. Viel schwieriger ist es, dem Verteilungsziel gerecht $z u$ werden, denn das Land sieht sich sofort mit der Frage konfrontiert, wie die Finanzmittel auf die einzelnen Kommunen aufgeteilt werden sollen. Wenn von $\mathrm{Z}$ uweisungen eine nivellierende Wirkung ausgehen soll, dann muß das Land, 
vereinfacht ausgedrückt, armen Gemeinden mehr geben als reichen. Wie dies im einzelnen konkret auszusehen hat, kann nicht allgemein gesagt werden, sondern hängt von der Art der zuweisung und ihrer zweckmäBigen Ausgestaltung ab.

\subsection{Zuweisungsarten}

Die Zuweisungen eines Landes an die Kommunen kann man nach der zweckbindung in allgemeine und zweckgebundene Zuweisungen einteilen. Allgemeine zuweisungen stellen für die Gemeinden genau wie die Steuern allgemeine Deckungsmittel dar und werden im Verwaltungshaushalt vereinnahmt. Den Kommunen ist somit freigestellt, wie sie die Zuweisungen verwenden wollen. Zweckgebundene zuweisungen sind dagegen mit Verwendungsauflagen verbunden.

Bei den allgemeinen Zuweisungen kann man im wesentlichen Schlüsselzuweisungen, mit denen wir es in dieser Arbeit hauptsächlich $z u$ tun haben, und Bedarfszuweisungen unterscheiden. Für Schlüsselzuweisungen steht vorab eine bestimmte Finanzmasse, die sogenannte Schlüsselmasse, zur Verfügung, die nach einem Schlüssel auf die Gemeinden verteilt wird. Danach erhält eine Gemeinde einen bestimmten Prozentsatz der Differenz zwischen ihrem Finanzbedarf und ihrer steuerkraft als Schlüsselzuweisung. Gemeinden, deren steuerkraft mindestens so hoch wie ihr Finanzbedarf ist, bekommen nichts. Gleichzeitig muß gewährleistet sein, daß alle Schlüsselzuweisungen zusammengenommen die vorhandene Schlüsselmasse genau ausschöpfen.

Die Bedarfszuweisungen, die im Vergleich $z u$ den Schlüssel$z$ uweisungen in allen Ländern quantitativ relativ unbedeutend sind, werden nur in Einzelfällen und auf Antrag gewährt. Dieser kann mit der auBergewöhnlichen Lage einer Kommune, etwa bei unvermeidlichen Haushaltsfehlbeträgen, oder mit 
"Härten" begründet sein, die bei der Verteilung der Schlüsselzuweisungen entstanden sind.

Bei den zweckgebundenen Zuweisungen gibt es eine ganze Reihe von zuweisungsarten. Sie sollen uns hier nicht weiter interessieren, wir führen kommentarlos nur einige Beispiele an. Es gehören dazu etwa Investitionszuweisungen, Erstattungen für Auftragsangelegenheiten, durchlaufende Gelder oder Zuweisungen für Sonderlastenausgleich. Durchlaufende Gelder sind Transferzahlungen von Bund und Land an natürliche Personen, die über die Gemeinden ausbezahlt werden. Zuweisungen für Sonderlastenausgleich sind z.B. Zahlungen für den Bau und Unterhalt von Straßen, mit denen die Gemeinden am Mineralölsteueraufkommen beteiligt werden.

Nach der Art und Weise der Verteilung unterscheidet man regelgebundene und nicht regelgebundene zuweisungen. Die Schlüsselzuweisungen sind, wie wir schon wissen, ein Beispiel für regelgebundene zuweisungen. Daneben gibt es im kommunalen Finanzausgleich noch andere regelgebundene $\mathrm{Zu}-$ weisungen, die aber nicht nach Finanzbedarf und Finanzkraft, sondern pauschal nach der Einwohnerzahl oder speziellen Bedarfskennziffern, etwa der Straßenlänge oder der Schülerzahl, verteilt werden. Nicht regelgebundene $\mathrm{z}$ uweisungen werden dagegen meist auf Antrag einer Gemeinde nach mehr oder weniger genau spezifizierten, nicht formalisierten Kriterien gewährt und räumen dem Land einen gewissen Ermessensspielraum ein.

\subsection{Finanzierung der Zuweisungen}

Die Finanzmasse, die dem Land zur Finanzierung der verschiedenen Zuweisungen zur Verfügung steht, setzt sich aus dem obligatorischen steuerverbund, dem fakultativen steuerverbund und aus Haushaltsmitteln des Landes zusammen. 
Der obligatorische Steuerverbund besteht aus dem Landesanteil an den Gemeinschaftssteuern, das sind die Einkommensteuer, Körperschaftsteuer und Umsatzsteuer. Diese finanziellen Mittel werden in einigen Ländern ergänzt durch die Einbeziehung weiterer Landessteuern (fakultativer Steuerverbund). Die Gemeinden erhalten von der so zusammengesetzten Verbundmasse einen bestimmten Prozentsatz (Verbundquote), der von Land zu Land verschieden ist, als zuweisungen. In einigen Ländern, so in Bayern, besteht abweichend davon der fakultative steuerverbund aus dem obligatorischen steuerverbund, von dem wieder ein bestimmter Prozentsatz den Gemeinden zusteht, und aus sogenannten Sonderverbünden einzelner Landessteuern, etwa der Mineralölsteuer, von denen den Gemeinden ein bestimmter Teil gesetzlich zusteht. $\mathrm{Zu}$ den gesamten Finanzausgleichsleistungen eines Landes an die Kommunen sind schließlich noch die zuweisungen zu zählen, die aus Haushaltsmitteln des Landes finanziert werden und die in den Finanzausgleichsgesetzen der Länder teilweise gar nicht vorgeschrieben sind.

Die Schlüsselmasse, also die finanziellen Mittel, die für Schlüsselzuweisungen zur Verfügung stehen, wird in allen Ländern dem Gemeindeanteil an der Verbundmasse entnommen. Allerdings ist dabei $z u$ beachten, daß von der Schlüsselmasse teilweise vorab bestimmte Mittel abgezogen werden. So verteilt sich in Rheinland-Pfalz ein Teil der Schlüsselmasse, die sogenannten Schlüsselzuweisungen B1, pauschal nach der Einwohnerzahl auf alle Gemeinden, wobei sich die ProKopf-Beträge der kreisfreien Städte, der großen kreisangehörigen städte und der Gemeinden geringfügig voneinander unterscheiden. In Baden-Württemberg erhalten alle Gemeinden vorab 20 DM je Einwohner. In Hessen gibt es für Gemeinden, die keine Schlüsselzuweisungen erhalten, sogenannte Mindestschlüsselzuweisungen (die nicht mit Sonderschlüsselzuweisungen und Mindestgarantien zu verwechseln sind), deren Pro-Kopf-Beträge ebenfalls gestaffelt sind. Schließlich 
werden in einigen Ländern auch die Sonderschlüsselzuweisungen der Schlüsselmasse vorab entnommen. Darauf werden wir noch ausführlich eingehen (siehe 2.2). Die verbleibende Finanzmasse wird dann in einigen Ländern nochmals auf Gemeinden und Gemeindeverbände aufgeteilt. Insgesamt kann man sagen, daB die gesetzlichen Regelungen in den Ländern hier sehr unterschiedlich sind. Wir wollen uns damit auch nicht weiter beschäftigen, für unsere Analyse genügt es zu wissen, daß für Schlüsselzuweisungen an Kommunen in jedem Land ein bestimmter Betrag, den wir im folgenden einfach "Schlüsselmasse" nennen, zur Verfügung steht und als gegeben anzusehen ist. 


\section{Das System der Schlüsselzuweisungen}

Der nun folgenden Darstellung des Systems der Schlüsselzuweisungen liegen die Finanzausgleichsgesetze der Bundesländer zugrunde (siehe Verzeichnis der Gesetze, Stand 1.1.1985).

\subsection{Die Schlüsselzuweisungen}

\subsubsection{Der Ausgleich zwischen Finanzbedarf und Steuerkraft}

Die Schlüsselzuweisungen an die Gemeinden errechnen sich in allen Bundesländern grob gesprochen aus einem Vergleich des Finanzbedarfs mit der Steuerkraft der Gemeinden. Danach erhält eine Gemeinde 1 eine (positive) Schlüsselzuweisung, wenn ihr Finanzbedarf $B_{i}$ gröBer als ihre steuerkraft $\mathrm{K}_{\mathrm{i}}$ ist. Dabei wird die Differenz zwischen Finanzbedarf und Steuerkraft $z u$ einem bestimmten Prozentsatz a > 0 , den man Ausgleichssatz oder Ausschüttungsquote nennt, ausgeglichen. In den meisten Bundesländern beträgt die Ausschüttungsquote gegenwärtig 50 , in Bayern 55 \& und im Saarland $80 \%$. 1) Ist der Finanzbedarf einer Gemeinde nicht größer als ihre Steuerkraft, dann erhält die Gemeinde keine Schlüsselzuweisung.

Bezeichnen wir mit $\underline{S}_{i}$ die Schlüsselzuweisung an Gemeinde $i$, dann berechnet sich $S_{i}$ also nach der Formel:

(2.1.1) $s_{i}= \begin{cases}0, & B_{i} \leqq K_{i} \\ a\left(B_{i}-K_{i}\right), & B_{i}>K_{i} .\end{cases}$

1) Für Baden-Württemberg kann kein fester Prozentsatz angegeben werden, da er von Jahr zu Jahr nach dem Verhältnis der Schlüsselmasse zu den Schlüsselzahlen neu bestimmt wird. Die Ausschüttungsquote betrug im Saarland bis zum Jahre $1983100 \%$. 
Als Maß für den Finanzbedarf einer Gemeinde dient die sogenannte AusgangsmeBzahl (BedarfsmeBzahl). Sie ist das Produkt aus dem Grundbetrag G, der in einem Land für alle Gemeinden gleich ist, und dem Gesamtansatz $E_{i}^{*}$ :

$$
\mathrm{B}_{i}:=\mathrm{E}_{i}^{*} \mathrm{G}
$$

Auf die Funktion und Berechnung des Grundbetrags werden wir noch ausführlich eingehen (siehe 2.1.2). Der Gesamtansatz ist eine mehrfach aus Hauptansatz und Nebenansätzen modifizierte Einwohnerzahl. Wie aus der tatsächlichen Einwohnerzahl $E_{i}$ einer Gemeinde $i$ die modifizierte Einwohnerzahl $E_{i}^{*}$ - ermittelt wird, ist in den einzelnen Bundesländern, wie wir gleich sehen werden, höchst unterschiedlich geregelt. Ganz allgemein gesprochen wird beim Hauptansatz die tatsächliche Einwohnerzahl mit einem bestimmten Prozentsatz angesetzt, der i.a. mit der Einwohnerzahl zunimmt. Durch Nebenansätze kann sich dieser Prozentsatz weiter erhöhen, oder es werden nach bestimmten Kriterien berechnete fiktive Einwohner zum Hauptansatz hinzugezählt.

Jedenfalls existiert für jede Gemeinde $i$ ein bestimmter Faktor $b_{i}$, der durch

$$
\mathrm{b}_{i}:=\frac{\mathrm{E}_{i}^{*}}{\mathrm{E}_{i}}
$$

eindeutig bestimmt ist, so daB sich die modifizierte Einwohnerzahl als Produkt aus der tatsächlichen Einwohnerzahl $E_{i}$ und $b_{i}$ darstellen läßt:

$$
E_{i}^{*}=E_{i} b_{i}
$$


Der Faktor $b_{i}$ für den Gesamtansatz setzt sich aus dem Faktor $w_{i}$ für den Hauptansatz und den Faktoren $v_{i j}$ für die Nebenansätze zusammen

$$
b_{i}=w_{i}+\sum_{j} v_{i j}
$$

wobei $v_{i j}$ den Nebenansatz $j$ in Gemeinde $i$ bezeichnet.

Der Faktor für den Hauptansatz ist in den meisten Bundesländern eine streng monoton wachsende Funktion w der Einwohnerzahl

$$
w: E_{i} \rightarrow w\left(E_{i}\right)=: w_{i}
$$

In Bayern etwa sieht $w$ folgendermaßen aus: ${ }^{1)}$

$$
100 \cdot w\left(\bar{E}_{i}\right)= \begin{cases}108+ & , \quad \bar{E}_{i} \leqq 5 \\ 108+1,4\left(\bar{E}_{i}-5\right) & , \quad 5<\bar{E}_{i} \leqq 10 \\ 115+0,66\left(\bar{E}_{i}-10\right) & , 10<\bar{E}_{i} \leqq 25 \\ 125+0,4\left(\bar{E}_{i}-25\right) & , 25<\bar{E}_{i} \leqq 50 \\ 135+0,1\left(\bar{E}_{i}-50\right) & , 50<\bar{E}_{i} \leqq 100 \\ 140+0,033\left(\bar{E}_{i}-100\right), 100<\bar{E}_{i} \leqq 250 \\ 145+0,02\left(\bar{E}_{i}-250\right), & , 250<\bar{E}_{i} \leqq 500 \\ 150+0,01\left(\bar{E}_{i}-500\right), & , 500<\bar{E}_{i}\end{cases}
$$

mit $\bar{E}_{i}=\frac{1}{1000} E_{i}$.

Dahinter steht die auf ${\underline{\mathrm{J} . \text { Popitz }^{2}}}^{2}$ und ${\underline{\mathrm{A} . \text { Brecht }^{3}}}^{\text {) }}$ zurück-

1) Die Multiplikation von w mit 100 bewirkt, daB auf der rechten Seite der Gleichung der Prozentsatz steht, mit dem die Einwohnerzahl angesetzt wird. Er wird vom Statistischen Landesamt nur auf eine Stelle hinter dem Komma berechnet.

2) Popitz [1932].

3) Brecht [1932]. 
gehende Vorstellung, ${ }^{1)}$ daß Gemeinden mit höherer Einwohnerzahl nicht nur einen absolut höheren Finanzbedarf als Gemeinden mit geringerer Einwohnerzahl haben, sondern auch einen höheren Finanzbedarf pro Einwohner. ${ }^{2)}$ Denn abstrahiert man von den Nebenansätzen und unterstellt

$$
\mathrm{b}_{i}=w\left(E_{i}\right)
$$

dann ist wegen der strengen Monotonie von $\mathrm{w}$ mit $\mathrm{G}>0$

$$
\frac{B_{i}}{E_{i}} \leqq \frac{B_{j}}{E_{j}}-G b_{i} \leqq G b_{j} \quad \omega w\left(E_{i}\right) \leqq w\left(E_{j}\right) \Leftrightarrow E_{i} \leqq E_{j} .
$$

Popitz hat in seinem Gutachten empirisch nachgewiesen, daB die Pro-Kopf-Ausgaben mit der Einwohnerzahl einer Kommune steigen. Dieser Zusammenhang wird dann (normativ) mit einem unterschiedlichen Bedarf verschieden großer Kommunen begründet. Anhand des Vergleichs einer kleinen ländlichen Gemeinde mit einer Großstadt oder kleineren Industriegemeinde kommt Popitz nämlich zu dem Schluß, daß die Einwohner in kleineren Gemeinden weniger bedürfen als die Einwohner gröBerer städte. ${ }^{3)}$ obwohl wir nicht präzisiert haben, was unter dem Bedarf einer Gemeinde überhaupt $z u$ verstehen ist, ${ }^{4)}$ wollen wir einmal annehmen, daß die Ausgaben einer Gemeinde nicht nur von ihrem Bedarf abhängen, sondern auch von den finanziellen Mitteln, die ihr zur Verfügung stehen. Dann kann es also sein, daß größere Gemeinden nur deshalb höhere Pro-Kopf-Ausgaben tätigen, weil sie pro Einwohner entweder eine größere Steuerkraft aufweisen oder aber höhere Zuwei-

1) Zur Entstehungsgeschichte der Hauptansatzstaffel siehe Hansmeyer, Kops [1985].

2) Popitz [1932], S. 266.

3) Popitz [1932], S. 279-281.

4) Siehe Hanusch, Kuhn [1985]. 
sungen erhalten. Höhere Zuweisungen wiederum werden, wie wir gesehen haben, mit steigenden Pro-Kopf-Ausgaben oder mit höherem Bedarf gerechtfertigt. Dann sind empirische Zusammenhänge zwischen Pro-Kopf-Ausgaben und Einwohnerzahl aber nur tautologisch, ${ }^{1)}$ oder sie bestätigen nur die von vornherein vorhandenen Werturteile.

Man könnte die Hauptansatzstaffel allenfalls mit den zentralörtlichen Funktionen größerer Gemeinden rechtfertigen. Bestimmte Gemeinden, die zentralen Orte, erstellen und finanzieren kollektive Güter, die nicht nur von den Einwohnern der zentralen orte selbst, sondern auch von den Einwohnern der Gemeinden im Verflechtungsbereich genutzt werden. Meist beteiligen sich die umliegenden Gemeinden nicht an den Produktionskosten der kollektiven Güter, so daß in den zentralen Orten gegenüber ihren Umlandgemeinden ein höherer Finanzbedarf angenommen werden kann. 2) Legt man die in der Raumordnung geläufige hierarchische struktur zentraler Orte zugrunde, wie sie etwa in Bayern im Landesentwicklungsprogramm festgelegt ist, so zeigt sich, da $B$ in Bayern (und wohl auch in anderen Bundesländern) Gemeinden der gleichen Zentralitätsstufe, d.h. mit den gleichen zentralörtlichen Funktionen, nicht immer der gleichen Größenklasse angehören. ${ }^{3)}$ schon allein deshalb ist die Hauptansatzstaffel zur Abgeltung zentralörtlicher Funktionen ungeeignet, ${ }^{4}$ ganz abgesehen von der Frage, ob die Prozentsätze des Hauptansatzes als ein MaB für. Bedarfsunterschiede zwischen Gemeinden mit unterschiedlichen zentralörtlichen Funktionen dienen können.

1) Vgl. Bös $[1971]$, S. 66 .

2) Zur Berücksichtigung zentralörtlicher Funktionen im kommunalen Finanzausgleich vgl. ausführlich: Hansmeyer [1980] und die dort angegebene Literatur, sowie unter allokativen Aspekten: Pollak, Voß [1985].

3) Siehe Hanusch, Kuhn [1985], S. 58.

4) Vgl. Hansmeyer [1980], S. 125. 
Deshalb ist die Hauptansatzstaffel in Schleswig-Holstein 1970 und in Rheinland-Pfalz 1978 abgeschafft und dafür $w\left(E_{i}\right)=1$ für alle Gemeinden $i$ angesetzt worden. Der Bedarf für zentralörtliche Funktionen wird in RheinlandPfalz in einem Nebenansatz berücksichtigt. In SchleswigHolstein gibt es auch keine Nebenansätze mehr, es wird bei den Schlüsselzuweisungen also von einem in allen Gemeinden einheitlichen Bedarf pro Einwohner ausgegangen, 1) d.h. es gilt

$$
b_{i}=1 \text { für alle } i
$$

In allen anderen Bundesländern gibt es meist mehrere Nebenansätze. ${ }^{2)}$ Damit wird der Finanzbedarf einer Gemeinde nicht nur von ihrer Einwohnerzahl abhängig gemacht, sondern auch von anderen Kennziffern des Bedarfs (Bedarfsindikatoren). 3) In den Nebenansätzen finden sich solche Kennziffern wie die geographische Lage einer Gemeinde, die demographische und soziale Zusammensetzung der Bevölkerung, das Bevölkerungswachstum, die Wirtschaftsstruktur, straßenlänge, Fremdenübernachtungen oder Schülerzahl. Die Nebenansätze führen faktisch durchweg zu einer weiteren (fiktiven) Erhöhung der Einwohnerzahl einer Gemeinde. Um dies zu illustrieren, betrachten wir beispielhaft den Raumordnungsansatz in Rheinland-Pfalz und den Ansatz für Bevölkerungswachstum in Bayern.

1) Zur Abgeltung der Leistungen für die Einwohner des Verflechtungsbereichs (nicht für die eigenen Einwohner) erhalten die zentralen Orte in Schleswig-Holstein allerdings zuweisungen aus einer eigenen Finanzmasse, der sogenannten Zentralitätsmasse.

2) Eine (neuere) Ubersicht findet sich in: Karrenberg, Münstermann [1983], S. $97 \mathrm{ff.;}$ siehe auch weber [1981], S. $110 \mathrm{ff}$. und Münstermann, Becker [1978], S. $35 \mathrm{ff}$.

3) Hanusch, Kuhn [1985], S. 59. 
Beim Ansatz für Bevölkerungszuwachs wird der Hauptansatz um ein Drittel des prozentualen Bevölkerungszuwachses der jeweils letzten 10 Jahre, höchstens jedoch um $\frac{1}{3}$ des Hauptansatzes erhöht. Bezeichne $v_{i 1}$ den Faktor für den Ansatz für Bevölkerungszuwachs in Gemeinde i, dann gilt

$$
v_{i 1}= \begin{cases}\frac{1}{3}\left(\frac{E_{i, 10}}{E_{i, 0}}-1\right) w_{i}, E_{i, 10} \leqq 2 E_{i, 0} \\ \frac{1}{3} w_{i}, \text { sonst, }\end{cases}
$$

wobei $E_{i, 0}$ die Einwohnerzahl in $i$ vor 10 Jahren und $E_{i, 10}$ die derzeitige Einwohnerzahl in $i$ ist mit $E_{i, 10} \geqq E_{i, 0^{\circ}}$

Beim Raumordnungsansatz in Rheinland-Pfalz werden zur Einwohnerzahl des Hauptansatzes rechnerisch weitere (fiktive) Einwohner hinzuaddiert: Unter- und Kleinzentren erhalten 3,5 v.H., Mittelzentren 2,5 v.H. und oberzentren 0,75 v.H. der Einwohnerzahl des Verflechtungsbereichs. Die Verflechtungsbereiche sind im Landesentwicklungsprogramm bzw. in den regionalen Raumordnungsplänen festgelegt und enthalten auch den zentralen ort.

Der Faktor $v_{i 2}$ für den Raumordnungsansatz eines zentralen ortes ist also definiert als

$$
v_{i 2}=P_{i} \frac{E_{i}^{v}}{E_{i}}
$$

mit $E_{i}^{v}$ : Einwohnerzahl im Verflechtungsbereich von $i$

$$
P_{i}= \begin{cases}0,035, & i \text { ist Unter- oder Kleinzentrum } \\ 0,025, & i \text { ist Mittelzentrum } \\ 0,0075, & i \text { ist Oberzentrum. }\end{cases}
$$


Wir können also festhalten: Die Berechnung des Finanzbedarfs der Gemeinden ist in den einzelnen Bundesländern sehr unterschiedlich. Abstrahiert man aber von diesen Unterschieden, so errechnet sich der Finanzbedarf einer Gemeinde in allen Ländern allgemein als

$(2.1 .2) \quad B_{i}:=b_{i} E_{i} G$

und der Finanzbedarf eines Einwohners in $i$ als

$$
\frac{B_{i}}{E_{i}}=b_{i} G
$$

Der Faktor $b_{i}$ läßt sich interpretieren als Wert eines Bedarfsindex

$$
\text { b: } \begin{aligned}
A & \rightarrow \mathbb{R} \\
i & \rightarrow b(i)=: b_{i},
\end{aligned}
$$

der jeder Kommune aus der Menge A aller Kommunen einen bestimmten Indexwert $b_{i}$ zuordnet.

Der Index hat die Interpretation

$$
b_{i} \leqq b_{j} \Leftrightarrow \begin{aligned}
& \text { der Bedarf eines Einwohners ist in } i \\
& \text { höchstens so großwie in } j, i, j \in A,
\end{aligned}
$$

denn es gilt $\left.\frac{B_{i}}{E_{i}} \leqq \frac{B_{j}}{E_{j}} \Leftrightarrow b_{i} \leqq b_{j} \cdot 1\right)$

Abschließend gehen wir noch kurz auf die steuerkraft einer Gemeinde ein. Sie errechnet sich in allen Bundesländern im wesentlichen als summe der Steuerkraftzahlen der Grundsteuer A

1) Vgl. ausführlich: Hanusch, Kuhn [1985], S. 59. 
und B, der Gewerbesteuer und dem Gemeindeanteil an der Einkommensteuer. Die Steuerkraftzahlen der Realsteuern erhält man durch Multiplikation der Meß- bzw. Grundbeträge mit fiktiven landeseinheitlichen Hundertsätzen, die auch als Nivellierungshebesätze bezeichnet werden. Auch der Gemeindeanteil an der Einkommensteuer wird in den meisten Bundesländern nicht mit $100 \%$, sondern mit einem geringeren prozentsatz angerechnet, so daß die tatsächlichen Einkommensteuereinnahmen der Gemeinden höher sind als es die Steuerkraftmeßzahl ausweist.

Seien also 1$)$

$T_{i j}:$ der MeBbetrag bzw. der Grundbetrag der steuer $j$ in Gemeinde $i$, $j=1, \ldots, 4$ (Grundsteuer A + B, Gewerbesteuer, Geme indeanteil an der Einkommensteuer),

$\mathrm{H}_{j}: \quad$ Nivellierungshebesatz bei der steuer $j$.

Daraus resultiert die steuerkraft

$$
\mathrm{K}_{i}=\sum_{j=1}^{4} \mathrm{H}_{j} \mathrm{~T}_{i j},
$$

die auch als normalisierte Steuerkraft bezeichnet wird, weil sie in der Regel von den tatsächlichen steuereinnahmen einer Gemeinde abweicht.

1) Vgl. Leibfritz, Teschner [1981], s. 316. 


\subsubsection{Die Ausschöpfung der Schlüsselmasse und das Versor- gungsniveau}

Die Finanzausgleichsgesetze der Länder unterscheiden sich, wie wir gesehen haben, insbesondere in der Berechnung des Finanzbedarfs erheblich voneinander. Die Schlüsselzuweisungen an die Gemeinden lassen sich aber durch Einsetzen von $(2.1 .2)$ in $(2.1 .1)$ auf die Grundstruktur

(2.1.3) $\quad S_{i}= \begin{cases}a\left(E_{i} b_{i} G-k_{i}\right), & , \frac{K_{i}}{E_{i} b_{i}} \\ 0 & \text {, sonst }\end{cases}$

reduzieren, wobei wir noch $b_{i}, E_{i}, k_{i}>0, i=1, \ldots, N$ und $0<a \leqq 1$ voraussetzen.

Der Grundbetrag ist die einzige endogene Variable, alle anderen Größen sind exogen bestimmt. Bezeichnet man den Bedarf eines für die Gemeinde i repräsentativen, d.h. mit ihrem Indexwert $b_{i}$ gewichteten Einwohners als normierten Finanzbedarf

(2.1.4) $\quad \bar{B}_{i}:=\frac{\mathrm{B}_{i}}{\mathrm{E}_{i} \mathrm{~b}_{i}}$,

dann läßt sich der Grundbetrag $\mathrm{G}$ interpretieren als normierter Finanzbedarf

$(2.1 .5) \quad \mathrm{G}=\overline{\mathrm{B}}_{\mathrm{i}} \quad$,

denn (2.1.5) ist äquivalent mit (2.1.2). Da (2.1.2) nur eine Definitionsgleichung ist, kann (2.1.5) zur Bestimmung von G nicht verwendet werden. Der Grundbetrag ist vielmehr so festzusetzen, daB die Gemeindeschlüsselmasse M, die bekanntlich exogen gegeben ist, mit Schlüsselzuweisungen genau ausgeschöpft wird. Sei $N \geqq 1$ die Anzahl der Gemeinden 
in einem Land, dann muß also gelten:

(2.1.6) $\quad \sum_{i=1}^{N} S_{i}=M \quad$ (Gleichgewichtsbedingung).

Der Grundbetrag sorgt sozusagen für ein Gleichgewicht zwischen Schlüsselmasse und der summe der Schlüsselzuweisungen. Bevor wir $(2.1 .3)$ in $(2.1 .6)$ einsetzen und daraus den Grundbetrag berechnen können, müssen wir die DummyVariable

$(2.1 .7) \quad \Theta_{i}(G)=\left\{\begin{array}{l}0, G \leqq \bar{K}_{i} \\ 1, G>\bar{k}_{i}, i=1, \ldots, N\end{array}\right.$

definieren, die den Wert 1 annimmt, wenn die Gemeinde $i$ eine Schlüsselzuweisung erhält und den Wert 0 sonst. Dabei ist analog zum normierten Finanzbedarf die normierte Steuerkraft

(2.1.8) $\quad \bar{k}_{i}:=\frac{k_{i}}{E_{i} b_{i}} \quad, \quad i=1, \ldots, N$

als Steuerkraft eines für die Gemeinde $i$ repräsentativen Einwohners definiert. Ob eine Gemeinde überhaupt Schlüsse1zuweisungen erhält, hängt also vom Vergleich ihrer normierten Steuerkraft mit dem Grundbetrag, also ihrem normierten Finanzbedarf ab. Da der Grundbetrag in allen Gemeinden gleich ist, erhalten alle Gemeinden, deren normierte steuerkraft kleiner als der Grundbetrag ist, (positive) Schlüsselzuweisungen, alle anderen Gemeinden erhalten keine. Dies werden wir uns später zunutze machen und die Gemeinden nach ihrer normierten steuerkraft in aufsteigender Reihenfolge ordnen. Es läßt sich in dieser ordnung dann die Ge- 
meinde mit derjenigen normierten Steuerkraft bestimmen, bis zu der alle Gemeinden Zuweisungen erhalten, deren normierte steuerkraft nicht größer ist.

Kommen wir zurück auf die Berechnung des Grundbetrags. Mit (2.1.7) können wir für (2.1.3) auch schreiben:

(2.1.9) $s_{i}=\theta_{i}(G) a\left(E_{i} b_{i} G-k_{i}\right), \quad i=1, \ldots, N$.

Eingesetzt in $(2.1 .6)$ folgt

(2.1.10) $\quad \sum_{i=1}^{N} \theta_{i}(G) a\left(E_{i} b_{i} G-K_{i}\right)=M$

und daraus ergibt sich

$(2.1 .11)$

$$
G=\frac{\frac{M}{a}+\sum_{i=1}^{N} \Theta_{i}(G) k_{i}}{\sum_{i=1}^{N} \Theta_{i}(G) E_{i} b_{i}}
$$

Dabei ist noch vorausgesetzt, $\mathrm{daB}$ es mindestens eine Gemeinde $i \in\{1, \ldots, N\}$ gibt mit $\Theta_{i}(G)=1$. Das ist aber, wie wir noch zeigen werden (s.5.1.1 und 2.3), immer dann der Fall, wenn $M>0$ ist. Es ist nun auch einsichtig, warum wir stets $a>0$ vorausgesetzt haben. Für $a=0$ ist (2.1.11) nicht definiert. Die Annahme der Existenz von $G$ würde sofort zum Widerspruch führen:

$$
\sum_{i=1}^{N} s_{i}=0 \neq M \quad \text { für } a=0, M>0 .
$$

Eine positive Schlüsselmasse käme bei $a=0$ also gar nicht zur Verteilung.

Betrachten wir nun (2.1.11) etwas genauer. Die Abhängig-

1) Vgl. Hanusch, Kuhn [1985], S. 60 . 
keit der $\theta_{i}$ vom Grundbetrag bedeutet, daß (2.1.11) nur in impliziter Form vorliegt und auch nicht explizit gemacht werden kann. Das zeigt sich darin, daß, salopp gesprochen, die Werte der Dummy-Variablen bekannt sein müBten, um den Grundbetrag berechnen zu können, andererseits aber die Dummy-Variablen nur dann bestimmte Werte annehmen, wenn der Grundbetrag bekannt ist. Dieses Problem wird in den Statistischen Landesämtern mit einem Iterationsverfahren ${ }^{1}{ }^{1}$ gelöst. Im Grunde werden dabei so lange Näherungslösungen berechnet, bis der Grundbetrag, also die Lösung von (2.1.10), gefunden ist. Bevor aber irgendein Iterationsverfahren überhaupt eingesetzt werden darf, ist zu prüfen, ob (2.1.10) eine Lösung hat und ob sie eindeutig ist. Wir wollen den Beweis dazu allerdings noch ein wenig zurückstellen ${ }^{2)}$ und zunächst einfach voraussetzen, $d a \beta$ ein eindeutiger Grundbetrag existiert.

Dann läßt sich (2.1.11) auch ohne Dummy-Variablen schreiben. Dazu ordnen wir, wie gesagt, die Gemeinden nach ihrer normierten steuerkraft in aufsteigender Reihenfolge, d.h. es gelte

$$
\overline{\mathrm{K}}_{1} \leqq \overline{\mathrm{K}}_{2} \leqq \cdots \leqq \overline{\mathrm{K}}_{\mathrm{N}}
$$

Aufgrund der Eindeutigkeit von $G$ existiert genau ein $\mathrm{n}(\mathrm{G}) \in\{1, \ldots, \mathrm{N}\}$, das für $\mathrm{G} \leqq \overline{\mathrm{K}}_{\mathrm{N}}$ durch

$$
\overline{\mathrm{K}}_{\mathrm{n}(\mathrm{G})}<\mathrm{G} \leqq \overline{\mathrm{K}}_{\mathrm{n}(\mathrm{G})+1}, \mathrm{n}(\mathrm{G}) \in\{1, \ldots, \mathrm{N}-1\}
$$

und furr $\mathrm{G}>\overline{\mathrm{K}}_{\mathrm{N}}$ durch

$$
\mathrm{n}(\mathrm{G})=\mathrm{N}
$$

1) Eine ausführliche (algorithmische) Beschreibung dieses Verfahrens geben wir in 2.4 an. Darüberhinaus schlagen wir zur derzeitigen Praxis auch alternative Verfahren vor (s. 2.4 und 5.1.1).

2) S. Abschnitt 2.3. 
bestimmt ist. ${ }^{1)}$

Die Zahl $n(G)$ gibt also die Nummer der Gemeinde in der Rangordnung der Gemeinden an, bis zu der alle Gemeinden Schlüsselzuweisungen erhalten. Gleichzeitig gibt sie die Zahl der zuweisungsberechtigten Gemeinden an. Damit gilt für die DummyVariablen

$$
\Theta_{i}(G)= \begin{cases}0, & i=n(G)+1, \ldots, N \\ 1, & i=1, \ldots, n(G)\end{cases}
$$

und für den Grundbetrag

$$
(2.1 .12) \quad G=\frac{\frac{M}{a}+\sum_{i=1}^{n(G)} K_{i}}{\sum_{i=1}^{n(G)} E_{i} b_{i}} .
$$

Die Gleichung (2.1.12) ist nur eine andere Form der Darstellung von (2.1.11) und (2.1.10). Der Grundbetrag ist hier nach wie vor nur implizit gegeben.

In der Literatur, auch der neueren, wird diese Gleichung jedoch häufig als eine explizite Darstellung des Grundbetrags interpretiert. 2) Das kommt daher, daß man in unzulässiger Weise von der Interdependenz zwischen der zahl der zuweisungsberechtigten Gemeinden $n(G)$ und dem Grundbetrag $G(n(G)$ ) abstrahiert und $n(G)$ einfach als bekannt annimmt. So findet sich in der Literatur für die Gleichung (2.1.12) öfter eine Darstellung, in der $\mathrm{n}(\mathrm{G})$ durch eine konstante $\mathrm{Zahl}$, meist $n$, ersetzt ist. Es kommt auch vor, daß die summationsgrenzen

1) Man beachte, der Fall $G \leqq \bar{K}_{1}$ und $\theta_{i}=0, i=1, \ldots, N$ kann für $M>0$ nicht eintreten, so daß $n(G) \geqq 1$ ist (siehe 2.3 und $5 \cdot 1 \cdot 1)$.

2) Siehe z.B. Münstermann [1975], S. 189; Gläser [1981], S. $296 \mathrm{f}$; Leibfritz, Teschner [1981], S. $\frac{.315}{\mathrm{ff}}$; Weber [1981], S. 124 f. 
überhaupt nicht spezifiziert sind ${ }^{1)}$, oder es wird nicht klar, ob über alle Gemeinden oder nur die zuweisungsberechtigten summiert werden soll. 2 )

Diese Art der Darstellung ist natürlich ungenau, sie erweckt dadurch jedoch leicht die Illusion, als wäre der Grundbetrag mit ihr explizit bestimmt. Tatsächlich muß sie in der Literatur dann auch häufig als Beweis für sehr weitreichende Behauptungen, etwa ubber die Abhängigkeit des Grundbetrags von einzelnen Variablen, wie der Schlüsselmasse oder Ausschüttungsquote, herhalten. ${ }^{3)}$ Dafür ist sie aber überhaupt nicht geeignet,ja, man kann vom mathematischen standpunkt aus behaupten, daß sie noch nicht einmal für eine implizite Beschreibung des Grundbetrags taugt.

Eine Veränderung etwa der Schlüsselmasse bewirkt, wie wir noch genau sehen werden, eine simultane Veränderung des Grundbetrags und der Zahl zuweisungsberechtigter Gemeinden. Dieser Zusammenhang kann mit Gleichung (2.1.12) nicht erfabt werden. Dazu würde man eine Darstellung benötigen, die den Grundbetrag von interessierenden Variablen funktional abhängig macht, und mit der der Grundbetrag auch explizit bestimmt wäre. Für dieses Problem gibt es in der Literatur bisher noch keine Lösung. Von daher wird auch verständlich, weshalb die Schlüsselzuweisungen mit formalanalytischen Methoden bisher noch nicht untersucht worden sind, denn dies hätte die analytische Bestimmung des gleichgewichtigen Grundbetrags notwendigerweise vorausgesetzt. ${ }^{4}$ )

1) Siehe etwa Weber [1981].

2) So z.B. bei Münstermann [1975] und Gläser [1981].

3) Siehe z.B. Weber [1981], S. 124 ; Münstermann [1975], S. $189 ;$ Gläser $[1981]$, S. 296.

4) Wir werden uns mit diesem Problem im Verlauf der Arbeit noch eingehend beschäftigen. 
Wir interpretieren den Grundbetrag nun noch ökonomisch. Durch Einsetzen von (2.1.12) in (2.1.2) und mit $n:=n(G)$ ergibt sich für den Finanzbedarf einer Gemeinde

$$
B_{i}=\frac{E_{i} b_{i}}{\sum_{j=1}^{n} E_{j} b_{j}}\left(\frac{M}{a}+\sum_{j=1}^{n} K_{j}\right)
$$

und damit ergibt sich

$$
\sum_{j=1}^{n} B_{j}=\frac{M}{a}+\sum_{j=1}^{n} k_{j} .
$$

Die Summe der Finanzbedarfe aller zuweisungsberechtigten Gemeinden entspricht also einem konstanten Betrag, den man als insgesamt verfügbare Finanzmittel interpretieren könnte, wenn man einen Ausgleichssatz von $a<1$ wie eine fiktive Erhöhung der Schlüsselmasse um den Faktor $\frac{1}{a}$ ansieht und die Steuerkraft der zuweisungsberechtigten Gemeinden hinzuaddiert. Der Finanzbedarf einer Gepeinde-ist dann ein Anteil an diesen Finanzmitteln, der sich nach dem Anteil der modifizierten Einwohnerzahl dieser Gemeinde an der Summe aller modifizierten Einwohnerzahlen bemiBt. Daran sieht man sehr schön, daß der Grundbetrag dafür sorgt, daB der Finanzbedarf der Gemeinden an die finanziellen Möglichkeiten angepaßt wira. Man könnte den Grundbetrag auch als durchschnittliches Versorgungsniveau interpretieren, wenn man den Finanzbedarf eines Einwohners in i nach Musgrave ${ }^{1)}$ definiert als

1) Musgrave $[1961]$, S. 100 . 
$(2.1 .13) \quad \frac{B_{i}}{E_{i}}:=b_{i} v_{i} \Rightarrow v_{i}=\frac{B_{i}}{E_{i} b_{i}}, i=1, \ldots, N$

$$
\begin{aligned}
\text { mit } \mathrm{b}_{i}: & \begin{aligned}
\text { index of need in } i \\
\text { (Bedarfsindexwert von } i),
\end{aligned} \\
v_{i}: & \text { level of performance in } i \\
& \text { (Versorgungsniveau in i). }
\end{aligned}
$$

Bei den Schlüsselzuweisungen wird das Versorgungsniveau in allen Gemeinden als gleich angenommen (siehe 2.1.2):

$$
v_{i}=G \text { für alle } i=1, \ldots, N
$$

Es entspricht daher stets dem durchschnittlichen Versorgungsniveau aller Gemeinden und ist so bemessen, da $\beta$ ein Gleichgewicht zwischen Zuweisungen und Finanzmitteln besteht. 1)

Versteht man im Gegensatz zu diesem Konzept unter dem Finanzbedarf einer Gemeinde etwa die exogen gegebenen Kosten der Bereitstellung öffentlicher Güter, so sind leicht Situationen denkbar, in denen die summe der Finanzbedarfe aller Gemeinden nicht den verfügbaren Finanzmitteln entspricht. Die Schlüsselmasse wäre also endogen so $\mathrm{zu}$ bemessen, daB die Differenz zwischen Finanzbedarf und Steuerkraft in allen Gemeinden mit der Ausschüttungsquote a ausgeglichen werden könnte. Dies könnte allerdings über den vorhandenen Verteilungsspielraum hinausgehen, falls die Steuereinnahmen für diese Schlüsselmasse nicht ausrelchen würden.

1) Bei Musgrave [1961, S. 101] sind gleiche Versorgungsniveaus erst das $\mathrm{Ziel}$ staatlicher Politik und Folge von Zuweisungen, wenn diese bestimmte Eigenschaften aufweisen (Musgrave's plan 2). Allerdings gewährleistet bei den Schlüsselzuweisungen ein gleiches Versorgungsniveau noch keine fiskalische Gleichheit (siehe Kapitel 4). 


\subsection{Sonderschlüsselzuweisungen und Mindestgarantien}

In allen Bundesländern beträgt derzeit

die Ausschüttungsquote weniger als $100 \%$. Es findet also nur ein teilweiser Ausgleich zwischen Finanzbedarf und Steuerkraft statt. In Gemeinden, deren Finanzbedarf größer als ihre steuerkraft ist, verbleibt daher auch nach der Aufstockung der eigenen Steuereinnahmen mit Schlüsselzuweisungen noch ein Finanzierungsdefizit (siehe 4.1). Deshalb gibt es in den Finanzausgleichsgesetzen Regelungen, um das Defizit zu beschränken. Sie unterscheiden sich erheblich voneinander. Es wird sich aber zeigen, daß sich für unsere Zwecke im wesentlichen zwei Verfahren unterscheiden lassen: Sonderschlüsselzuweisungen und Mindestgarantien.

\subsubsection{Sonderschlüssel zuweisungen}

Manche Bundesländer gewähren Sonderschlüsselzuweisungen, deren Höhe sich im wesentlichen aus einem Vergleich der Steuerkraft einer Gemeinde mit der landesdurchschnittlichen Steuerkraft bemißt. Die Mittel für Sonderschlüsselzuweisungen werden der Schlüsselmasse vorab entnommen.

In Bayern etwa erhalten "Gemeinden, deren Steuerkraftmeßzahl je Einwohner unter $75 \mathrm{v} . \mathrm{H}$. des mit dem Vomhundertsatz ihres Hauptansatzes angesetzten Landesdurchschnitts bleibt, . $引$; $15 \mathrm{v} . \mathrm{H}$. des Unterschieds als Sonderschlüsselzuweisung." 1 )

Bezeichne $a_{e}(=75 \%)$ den sogenannten Eingriffssatz und $a_{\text {s }}(=15$ v.H.) den Ausgleichssatz für Sonderschlüsselzuweisungen, dann berechnet sich die Sonderschlüsselzuwei-

1) Bayerisches Finanzausgleichsgesetz, $§ 3(2)$. 
weisung $s_{i}^{s}$ an Gemeinde $i$ wie folgt ${ }^{1)}$ :

$$
s_{i}^{S}= \begin{cases}0 & , \frac{K_{i}}{E_{i}} \geqq \frac{\sum_{j=1}^{N} K_{j}}{j=1} w_{i} a_{j} \\ \left(\frac{\sum_{j=1}^{N} K_{j}}{\sum_{j=1}^{N} E_{j}}-\frac{K_{i}}{E_{i}}\right) a_{s} E_{i}, & \text { sonst. }\end{cases}
$$

Definiert man eine Dummy-Variable

$$
\Phi_{i}= \begin{cases}0, & \frac{k_{i}}{E_{i} w_{i}} \geq \frac{\sum_{j=1}^{N} K_{j}}{\sum_{j=1}^{N} E_{j}} a_{e} \\ 1, & \text { sonst },\end{cases}
$$

dann läßt sich $S_{i}^{S}$ auch so schreiben:

$$
s_{i}^{s}=\Phi_{i}\left(\frac{\sum_{j=1}^{N} K_{j}^{N}}{\sum_{j=1}^{N} E_{j}}-\frac{K_{i}}{E_{i} w_{i}}\right) a_{s} E_{i} w_{i} .
$$

Man sieht, ob eine Gemeinde überhaupt Sonderschlüsselzuweisungen erhält, hängt $a b$ von einem Vergleich ihrer steuerkraft, die auf einen mit dem Hauptansatz gewogenen Einwoh-

1) Vgl. Leibfritz, Teschner [1981], S. 316. 
ner bezogen ist, mit einer für alle Gemeinden gleichen Größe. Dieser Vergleich ist aber nicht sehr aussagefähig, weil der Quotient $\sum_{i} K_{i} / \Sigma_{i} E_{i}$ gar nicht als landesdurchschnittliche Steuerkraft interpretiert werden kann. Es gibt nämlich keine Funktion

$$
f:\left(\frac{K_{1}}{E_{1}}, \ldots, \frac{K_{N}}{E_{N}}\right) \rightarrow \frac{\sum_{i=1}^{N} K_{i}}{\sum_{i=1}^{N} E_{i}}
$$

für alle $K_{i}, E_{i}>0$,

die es gestatten würde, diesen Quotienten als Aggregat der einwohnerbezogenen steuerkraftzahlen darzustellen.

Angenommen, es gäbe eine solche Funktion.

Für den Spezialfall $E_{1}=E_{2}=\ldots=E_{N}=1$ müßte

$$
f\left(\frac{K_{1}}{E_{1}}, \ldots, \frac{K_{N}}{E_{N}}\right)=\frac{1}{N} \sum_{i=1}^{N} K_{i}
$$

sein. Allgemein wäre $f$ also das arithmetische Mittel der einwohnerbezogenen Steuerkraftzahlen

$$
f\left(\frac{K_{1}}{E_{1}}, \ldots, \frac{K_{N}}{E_{N}}\right)=\frac{1}{N} \sum_{i=1}^{N} \frac{K_{i}}{E_{i}}, K_{i}>0, E_{i}>0 .
$$

Im allgemeinen ist aber 


$$
\frac{\sum_{i=1}^{N} K_{i}}{\sum_{i=1}^{N} E_{i}} \neq \frac{1}{N} \sum_{i=1}^{N} \frac{K_{i}}{E_{i}}
$$

und somit ist die Annahme der Existenz von f falsch.

1)

Man kann durch geeignete Beispiele sogar zeigen, daß ein Steigen aller einwohnerbezogenen Steuerkraftzahlen zu einem Sinken der Größe $\Sigma_{i} K_{i} / \Sigma_{i} E_{i}$ führen kann. Entsprechend kann das Sinken aller Steuerkraftzahlen $z u$ einem Steigen dieser Größe führen. 2 )

Auch wenn $\Sigma_{i} K_{i} / \Sigma_{i} E_{i}$ sich nicht als landesdurchschnittliche Steuerkraft interpretieren läßt, so kann man dennoch den Kreis der Gemeinden angeben, die Sonderschlüsselzuweisungen erhalten.

Seien die Gemeinden in der Reihenfolge

$$
\frac{K_{1}}{E_{1} w_{1}} \leqq \cdots \leqq \frac{K_{N}}{E_{N} w_{N}}
$$

numeriert, und sei $m \in\{1, \ldots, N-1\}$ durch

$$
\frac{k_{m}}{E_{m} w_{m}}<\frac{\sum_{i=1}^{N} K_{i}}{\sum_{i=1}^{N} E_{i}} a_{e} \leqq \frac{k_{m+1}}{E_{m+1} w_{m+1}}
$$

1) Einen analogen Beweis hat zuerst Stehling [1983, S. $197 \mathrm{f.}]$ für den (funktionalen) Zusammenhang von einzel- und gesamtwirtschaftlichen Arbeitsproauktivitäten geführt.

2) Vgl. den analogen Beweis von stehling [1983], S. $198 \mathrm{f}$. 
bestimmt. 1) Dann ist

$$
\begin{aligned}
& \Phi_{i}=1 \text { für } i=1, \ldots, m \\
& \Phi_{i}=0 \text { für } i=m+1, \ldots, N^{2)},
\end{aligned}
$$

und $\mathrm{m}$ gibt die Gemeinde in der Rangordnung an, die gerade noch Sonderschlüsselzuweisungen erhält.

Die für Sonderschlüsselzuweisungen insgesamt benötigten Mittel betragen:

$$
\begin{aligned}
\sum_{i=1}^{N} s_{i}^{s} & =a_{s} \frac{\sum_{i=1}^{N} E_{i}^{N}}{\sum_{i=1}^{N}} \sum_{i}^{N} E_{i} w_{i}-a_{s} \sum_{i=1}^{N} \Phi_{i} k_{i} \\
& =a_{s}\left(\frac{\sum_{i=1}^{N} K_{i}}{\sum_{i=1}^{N} E_{i}} \sum_{i=1}^{m} E_{i} w_{i}-\sum_{i=1}^{m} K_{i}\right) .
\end{aligned}
$$

Bezeichnet man mit

1) Falls $\frac{K_{N}}{E_{N} W_{N}}<\frac{\sum K_{i}}{\sum E_{i}} a_{e}$ ist, dann ist $m=N$.

Notwendige Voraussetzung dafür ist

$$
a_{e^{w_{N}}}>1 \Rightarrow w_{N}>\frac{1}{a_{e}} \text {, }
$$

denn es gilt stets $\frac{\sum \mathrm{K}_{i}}{\sum \mathrm{E}_{\mathrm{i}}} \leqq \frac{\mathrm{K}_{\mathrm{N}}}{\mathrm{E}_{\mathrm{N}}}$.

In der Regel dürfte aber $m<N$ sein.

2) Angenommen wird $\Phi_{i}=1$ für mindestens ein $i$ und folglich $\mathrm{m} \geqq 1$. 


$$
s_{i}^{g}:=s_{i}^{s}+s_{i}
$$

die Summe aus Schlüsselzuweisung und Sonderschlüsselzuweisung der Gemeinde $i$ und mit

$$
M g:=\sum_{i=1}^{N} s_{i}^{g}
$$

die für Sonderschlüsselzuweisungen und Schlüsselzuweisungen insgesamt zur Verfügung stehende Finanzmasse, dann ergibt sich die für Schlüsselzuweisungen verbleibende Schlüsselmasse $M$ als

$$
\text { (2.2.1) } M=M g-\sum_{i=1}^{N} s_{i}^{s} \text {, }
$$

denn durch Einsetzen obiger Definitionscleichunçen in (2.2.1) folgt sofort

$$
M=\sum_{i=1}^{N} s_{i}
$$

Charakteristisch für dieses Verfahren ist also, daß die Mittel für Sonderschlüsselzuweisungen der Schlüsselmasse vorab entnommen werden können. Dies ist deshalb möglich, weil die Sonderschlüsselzuweisung einer Kommune nicht von ihrem Finanzbedarf und somit auch nicht vom Grundbetrag abhängt. Die zuweisungsberechtigten Gemeinden und die Sonderschlüsselzuweisungen, die sie beanspruchen können, stehen fest und ändern sich auch nicht, wenn sich etwa die Finanzmittel Mg ändern. Der Grundbetrag G wird in diesem System nun so festgesetzt, $d a B$ die nach Abzug der Sonderschlüsselzuweisungen verbleibende Schlüsselmasse $M$ ausgeschöpft wird (wie wir es in 2.1 .2 beschrieben haben).

Vergleichbare Regelungen gibt es auch in anderen Bundesländern. 
In Rheinland Pfalz erhalten Ortsgemeinden, verbandsfreie Gemeinden, große kreisangehörige städte und kreisfreie Städte, deren SteuerkraftmeBzahl weniger als $74 \mathrm{v} . \mathrm{H}$. des Landesdurchschnitts beträgt, vorweg, d.h. vor der Verteilung der sogenannten Schlüsselzuweisungen B, den Unterschledsbetrag als Schlüsselzuweisung A. Im Saarland erhalten die Gemeinden entsprechend $70 \&$ des Unterschiedsbetrages. In Baden-Württemberg gibt es für Gemeinden, deren Steuerkraftmeßzahl unter $50 \mathrm{v} \cdot \mathrm{H}$. der Ausgangsmeßzah ${ }^{1}{ }^{1}$ liegt, Mehrzuweisungen in Höhe des Unterschieds.

\subsubsection{Mindestgarantien}

Bei der Mindestgarantie (oder Sockelgarantie) muß die Summe aus Schlüsselzuweisung und steuerkraft einen bestimmten Prozentsatz as (Garantiesatz) der Ausgangsmeßzahl betragen, so in Hessen $\left(a_{s}=75 \%\right)$, Niedersachsen $\left(a_{s}=80 \%\right)$ und Nordrhein-Westfalen $\left(a_{s}=90 \%\right)$. Obwohl in diesen Ländern also keine gesonderten Sonderschlüsselzuweisungen gewährt werden, ist es für die formale Darstellung (und zum Vergleich mit der in 2.2.1 beschriebenen Regelung) zweckmäßig, sich die gesamte Schlüsselzuweisung $s_{i}^{g}$ wieder als summe einer (fiktiven) Sonderschlüsselzuweisung $s_{i}^{s}$ und einer Schlüsselzuweisung $S_{i}$ vorzustellen:

$$
s_{i}^{g}:=s_{i}^{s}+s_{i}
$$

Dabei ist $S_{i}$ wie üblich durch

1) In Baden-Württemberg ist die Ausgangsmeßzahl vom Grundbetrag unabhängig. 


$$
\begin{aligned}
s_{i} & =\Theta_{i} a\left(E_{i} b_{i} G-K_{i}\right) \\
\theta_{i} & =\left\{\begin{array}{cc}
0, & \bar{k}_{1} \geqq G \\
1, & \bar{k}_{i}<G
\end{array}\right. \\
i & =1, \ldots, N
\end{aligned}
$$

bestimmt (siehe $(2.1 .9)$ und $(2.1 .7)$ ).

Die Mindestgarantie stellt sicher, daß die Bedingung

$$
\text { (2.2.3) } s_{i}^{g}+k_{i}=s_{i}^{S}+s_{i}+k_{i} \geqq a_{s} E_{i} b_{i} G
$$

immer erfüllt ist.

Falls also

$$
s_{i}+K_{i} \geqq a_{s} E_{i} b_{i} G
$$

gilt, dann ist $s_{i}^{S}=0$ und $s_{i}^{g}=s_{i}$.

Falls aber

$$
s_{i}+k_{i}<a_{s} E_{i} b_{i} G
$$

ist, dann resultieren aus $(2.2 .3)$ Sonderschlüsselzuweisungen

$$
s_{i}^{s}=a_{s} E_{i} b_{i} G-s_{i}-k_{i} \text {. }
$$

Dafür kann man zusammenfassend schreiben:

(2.2.4) $s_{i}^{s}=\left\{\begin{array}{l}0, s_{i} \geqq a_{s} E_{i} b_{i} G-K_{i} \\ a_{s} E_{i} b_{i} G-s_{i}-k_{i}, \text { sonst. }\end{array}\right.$

Definiert man die Dummy-Variable 
(2.2.5) $\Psi_{i}:=\left\{\begin{array}{l}0, s_{i} \geqq a_{s} E_{i} b_{i} G-K_{i} \\ 1, \text { sonst, }\end{array}\right.$

dann erhält man

(2.2.6) $\quad S_{i}^{S}=\Psi_{i}\left(a_{s_{i}} E_{i} b_{i}-s_{i}-K_{i}\right)$.

Setzt man (2.2.2) in (2.2.5) und (2.2.6) ein, so ergibt sich

(2.2.7) $\Psi_{i}=\left\{\begin{array}{l}0, \frac{1-\theta_{i} a}{1-x_{i}} \\ 1, \text { sonst }\end{array}\right.$

und

(2.2.8) $\quad s_{i}^{s}=\Psi_{i}\left(a_{s}-\Theta_{i} a\right) E_{i} b_{i} G-\Psi_{i}\left(1-\Theta_{i} a\right) k_{i}, i=1, \ldots, N$.

Nun können wir auch angeben, welche Werte $a_{s}$ sinnvollerweise nur annehmen kann.

Sicher muß $a_{s}>a$ sein, denn für $a_{s} \leqq a$ folgt aus (2.2.7)

$$
\Psi_{i}=0 \text { für } \theta_{i}=1 \vee \theta_{i}=0 \text {, }
$$

d.h. keine Gemeinde würde Sonderschlüsselzuweisungen erhalten, weil der Garantiesatz zu niedrig ist.

Andererseits muß $a_{s}<1$ gefordert werden, denn es wäre

$$
\Psi_{i} \equiv \theta_{i} \quad \text { für } a_{s}=1 \text {, }
$$

und alle schlüsselzuweisungsberechtigten Gemeinden erhielten auch Sonderschlüsselzuweisungen. Die gesamte Schlüssel- 
zuweisung einer Gemeinde wäre

$$
s_{i=1, \ldots, N}^{g} \begin{cases}E_{i} b_{i} G-k_{i}, & \\ 0 & \text { sonst }\end{cases}
$$

und wïrde zusammen mit der steuerkraft immer mindestens den Bedarf decken:

$$
s_{i}^{g}+k_{i} \geqq B_{i} \quad, \quad i=1, \ldots, N \text {. }
$$

Dieses Resultat ist aber einfacher mit einer Ausschüttungsquote von $\mathrm{a}=1 \mathrm{zu}$ erzielen, wie dies im Saarland als einzigem Bundesland auch tatsächlich der Fall war. Von daher wird auch verständlich, warum dort auf Mindestgarantien oder Sonderschlüsselzuweisungen bis zum Jahr 1983 verzichtet werden konnte (siehe auch Kapitel 7).

Bel $a_{s}>1$ würde gelten:

$$
\Psi_{i}=1 \text { für } \theta_{i}=1 \vee\left(\theta_{i}=0 \wedge a_{s} G>\bar{k}_{i}\right)
$$

Elnige Gemeinden, die keine Schlüsselzuweisungen erhalten, weil sie schon einen UberschuB ihrer eigenen Steuereinnahmen über den Finanzbedarf aufweisen (abundante Gemeinden) erhielten sogar Sonderschlüsselzuweisungen. 1) Alle Gemeinden würden dann einen FinanzierungsüberschuB aufweisen.

Im folgenden wollen wir stets $\mathbf{a} \leqq a_{\mathbf{s}}<1$ voraussetzen. In diesem Bereich liegen auch die gesetzlichen Garantiesätze.

1) Tatsächlich gibt es in einigen Bundesländern wie wir wissen (siehe Kapitel 1) sogar Zuweisungen für abundante Gemeinden. Dazu gehören die genannten Kopf-Beträge in Baden-Württemberg und Rheinland-Pfalz und die Mindestschlüsselzuweisungen in Hessen. 
Dann erhält eine Gemeinde nur dann Sonderschlüsselzuweisungen, wenn sie auch schlüsselzuweisungen erhält, denn es gilt

$$
G\left(\frac{a_{s}-\theta_{i} a^{a}}{1-\theta_{i}^{a}}\right)>\bar{K}_{i} \rightarrow G>\bar{K}_{i}
$$

und folglich

(2.2.9) $\Psi_{i}=1 \Rightarrow \Theta_{i}=1$.

Die Umkehrung gilt nicht, d.h. eine Gemeinde, die Schlüsselzuweisungen erhält, muß nicht immer Sonderschlüsselzuweisungen bekommen.

Andererseits gilt

$$
\bar{k}_{i} \geqq G \quad \Rightarrow \quad \bar{k}_{i} \geqq G\left(\frac{a_{s}-\theta_{i} a^{-}}{1-\theta_{i}^{a}}\right)
$$

und somit

(2.2.10) $\theta_{i}=0 \Rightarrow \Psi_{i}=0$.

Abundante Gemeinden können keine Sonderschlüsselzuweisungen beanspruchen. Das besagt aber nicht, daß Gemeinden, die keine Sonderschlüsselzuwelsungen erhalten, auch nicht schlüsselzuweisungsberechtigt sind.

Aus der Addition von (2.2.8) und (2.2.4) und einigen Umformungen folgt

$$
s_{i}^{g}=\Psi_{i}\left(a_{s} E_{i} b_{i} G-K_{i}\right)+\left(1-\Psi_{i}\right) \theta_{i} a\left(E_{i} b_{i} G-K_{i}\right)
$$

bzw.

$$
\text { (2.2.11) } s_{i}^{g}=\left\{\begin{array}{l}
a_{s} E_{i} b_{i} G-k_{i}, \Psi_{i}=1 \\
\Theta_{i} a\left(E_{i} b_{i} G-k_{i}\right), \Psi_{i}=0 .
\end{array}\right.
$$


Die gesamte Schlüsselzuweisung einer Gemeinde ist also gleich der Differenz zwischen dem $a_{s}$-fachen des Finanzbedarfs und der steuerkraft, wenn die Mindestgarantie wirksam wird, ansonsten entspricht sie $S_{i}$.

Der Grundbetrag ist bei diesem Verfahren so festzusetzen, daß die für Schlüsselzuweisungen $S_{1}^{g}$ (also einschließlich der Mindestgarantien) insgesamt verfügbare Schlüsselmasse 'Mg ausgeschöpft wird:

$$
\sum_{i=1}^{N} s_{i}^{g}=M g
$$

Durch Einsetzen von (2.2.11) resultiert daraus: ${ }^{1)}$

$(2.2 .13)$

$$
G=\frac{M g+\sum_{i=1}^{N}\left(\left(1-\Psi_{i}\right) \Theta_{i} a+\Psi_{i}\right) k_{i}}{\sum_{i=1}^{N} E_{i} b_{i}\left(\left(1-\Psi_{i}\right) \Theta_{i} a+\Psi_{i} a_{s}\right)} .
$$

Wir nehmen an, Gleichung $(2.2 .12)$ habe eine eindeutige Lösung und die Gemeinden seien nach ihrer normierten Steuerkraft geordnet. Dann existiertein $\left.k:=k(G) \in\{1, \ldots, N\}^{2}\right)$ mit

$$
\Psi_{i}(G)= \begin{cases}0, & i=k+1, \ldots, N \\ 1, & i=1, \ldots, k\end{cases}
$$

1) Man beachte, G liegt nicht in expliziter Form vor, denn sowohl $\Psi_{i}$ (und damit die Sonderschlüsselzuweisungen) als auch $\Theta_{i}$ hängen von $G$ ab.

2) Es kann auch $k=0$ sein, falls $\Psi_{i}=0, i=1, \ldots, N$ ist, d.h. keine Gemeinde Sonderschlüsselzuweisungen erhält. Wir wollen aber $\Psi_{i}=1$ für mindestens ein $i$ und $k \geqq 1$
annehmen. 
und ein $1:=1(G) \in\{1, \ldots, N\}^{1)}$ mit

$$
\Theta_{i}(G)= \begin{cases}0, & i=1+1, \ldots, N \\ 1, & i=1, \ldots, 1 .\end{cases}
$$

Dabei gilt mit (2.2.9) $1 \geqq k$. Gleichung (2.2.13) kann nun in der Form

$$
G=\frac{M g+\sum_{i=1}^{k} k_{i}+\sum_{i=k+1}^{1} a k_{i}}{\sum_{i=1}^{k} a{ }_{s} E_{i} b_{i}+\sum_{i=k+1}^{1} a E_{i} b_{i}}
$$

geschrieben werden.

Charakteristisch für Mindestgarantien ist die Abhängigkeit der (fiktiven) Sonderschlüsselzuweisungen vom Grundbetrag, der wiederum von Mg abhängt. Deren Höhe bestimmt sich nur simultan mit den Schlüsselzuweisungen, und sie können deshalb auch nicht vorab der Schlüsselmasse entnommen werden. Man kann deshalb erst ex-post sagen, welcher Teil von Mg für die Mindestgarantie (bzw. für fiktive Sonderschlüsselzuweisungen) und welcher für Schlüsselzuweisungen aufgewendet wird. In der Praxis wird ja bekanntlich so vorgegangen, daß zwischen Sonderschlüsselzuweisungen und Schlüsselzuweisungen nicht differenziert wird. Eine Gemeinde bekommt stattdessen die Zuweisung $s_{i}^{g}$, bei der ein bestimmter Mindestbetrag garantiert ist.

In Schleswig-Holstein bekommen die Gemeinden Sonderschlüsselzuweisungen, und zwar das $a_{e}$-fache $\left(a_{e}=0,5\right)$ des Unterschieds zwischen einem bestimmten Prozentsatz a (bis zu

1) Für Mg > 0 gibt es mindestens ein i mit $\Theta_{i}=1$. 
80 8) des Finanzbedarfs und der Steuerkraft

$$
s_{i}^{s}=a_{e}\left(a_{s} E_{i} b_{i} G-k_{i}\right) \text {, falls } a_{s} E_{i} b_{i} G>K_{i} \text {. }
$$

Definiert man

$$
\Omega_{i}:=\left\{\begin{array}{cc}
0, & \overline{\mathrm{k}}_{i} \geqq a_{s} \mathrm{G} \\
1, & \text { sonst }
\end{array}\right.
$$

dann schreibt sich

$$
s_{i}^{s}=\Omega_{i} a_{e}\left(a_{s} E_{i} b_{i} G-k_{i}\right), i=1, \ldots, N .
$$

Damit erhält man

$$
s_{i}^{g}=\left(\Omega_{i} a_{e} a_{s}+\theta_{i} a\right) E_{i} b_{i} G-\left(\Omega_{i} a_{e}+\theta_{i} a\right) k_{i} .
$$

Die Bedingung

$$
\sum_{i=1}^{N} s_{i}^{g}=M g
$$

liefert für den Grundbetrag

$$
G=\frac{M g+\sum_{i=1}^{N}\left(\Omega_{i} a_{e}+\Theta_{i} a\right) k_{i}}{\sum_{i=1}^{N}\left(\Omega_{i} a_{e} a_{s}+\Theta_{i} a\right) E_{i} b_{i}}
$$

Die Sonderschlüsselzuweisungen bewirken, daß das Defizit zwischen Bedarf und Steuerkraft zu einem höheren Prozentsatz als a ausgeglichen wird, falls $\Omega_{i}=1$ ist. Obwohl in Schleswig-Holstein Sonderschlüsselzuweisungen separat von Schlüsselzuweisungen gewährt werden, ordnen wir sie den Mindestgarantien $z u$, weil sie genau wie jene vom Grundbe- 
trag abhängen und nicht vorab der Schlüsselmasse entnommen werden können.

Wir werden uns mit Sonderschlüsselzuweisungen und Mindestgarantien erst wieder in Kapitel 7 beschäftigen. Bis dahin abstrahieren wir von ihnen und betrachten nur Schlüsselzuweisungen. Wenn nachfolgend von der Schlüsselmasse $M$ die Rede ist, so ist damit eine Schlüsselmasse gemeint, die vorab um Sonderschlüsselzuweisungen gekürzt wurde, also ausschließlich für Schlüsselzuweisungen verwendet wird.

\subsection{Existenz und Eindeutigkeit der Schlüsselzuweisungen}

In diesem Abschnitt wird gezeigt, daß der Grundbetrag existiert und eindeutig ist, was wir bisher stets vorausgesetzt haben. Dann sind auch die Schlüsselzuweisungen an die Gemeinden eindeutig, denn alle anderen Größen, die in ihre Berechnung noch eingehen, sind gegeben. Bei dem Beweis ist wieder vorausgesetzt, daß die Gemeinden nach ihrer normierten steuerkraft geordnet sind.

\section{Beweis:}

Wir gehen von der Identität

$$
\sum_{i=1}^{N} s_{i}=M
$$

aus. Benutzt man für die Schlüsselzuweisungen statt (2.1.9) den äquivalenten Ausdruck

$$
\text { (2.3.2) } \begin{aligned}
s_{i} & =\Theta_{1} a E_{i} b_{i}\left(G-\bar{K}_{i}\right) \text { mit } \\
\theta_{i} & =\left\{\begin{array}{cc}
0, & G \leqq \bar{K}_{i} \\
1, & G>\bar{K}_{i} .
\end{array}\right.
\end{aligned}
$$


so erhält man nach Einsetzen von (2.3.2) in (2.3.1)

(2.3.3) $\quad \sum_{i=1}^{N} \theta_{i} E_{i} b_{i}\left(G-\bar{K}_{i}\right)=\frac{M}{a}$.

Interpretiert man die linke Selte von Gleichung (2.3.3) als Funktion

$(2.3 .4) \quad S: G+S(G):=\sum_{i=1}^{N} \Theta_{i} E_{i} b_{i}\left(G-\bar{K}_{i}\right)$,

dann gibt es für ein beliebiges $\operatorname{Paar}(M, a) \in \mathbb{R}_{++} \times \mathbb{R}_{++}{ }^{1)}$ jeweils ein eindeutiges $\mathrm{G}$ mit

$$
S(G)=\frac{M}{a} \text {, }
$$

das also Lösung von (2.3.3) ist, falls der Wertebereich von $S$ alle positiven reellen zahlen umfaßt und falls $s$ für alle $\mathrm{G}>\mathrm{O}$ streng monoton ist.

Durch Elimination $\operatorname{der} \theta_{i}(i=1, \ldots, N)$ erkennt man, daB $S$ abschnittsweise definiert ist:

$(2.3 .5) \quad S(G)= \begin{cases}0 & , G \in\left(0, \bar{K}_{1}\right] \\ \sum_{i=1}^{n} E_{i} b_{i}\left(G-\bar{K}_{i}\right), & G \in\left(\bar{K}_{n}, \bar{K}_{n+1}\right), \\ & n=1, \ldots, N-1 \\ \sum_{i=1}^{N} E_{1} b_{i}\left(G-\bar{K}_{1}\right), G>\bar{K}_{N} .\end{cases}$

Klar ist, in den Intervallen $\left(\bar{K}_{n}, \bar{K}_{n+1}\right), n=1, \ldots, N-1$ und

1) $\mathbb{R}_{++}=\{x \in \mathbb{R} \mid x>0\}$ 
für $G>\bar{K}_{N}$ ist $S$ stetig und streng monoton wachsend mit den Steigungen

$$
\frac{\mathrm{dS}(G)}{\mathrm{dG}}=\sum_{i=1}^{n} E_{i} b_{i}>0, \quad n=1, \ldots, N-1
$$

bzw.

$$
\frac{\mathrm{dS}(G)}{\mathrm{dG}}=\sum_{i=1}^{N} \mathrm{E}_{i} \mathrm{~b}_{i}>0, \quad G>\overline{\mathrm{K}}_{\mathrm{N}} .
$$

Im Intervall $\left(0, \overline{\mathrm{K}}_{1}\right)$ ist $\mathrm{S}(\mathrm{G})=0$, da $\theta_{i}=0$ und folglich $s_{i}=0$ ist für alle $i=1, \ldots, N$. In diesem Intervall kann die Lösung also nicht liegen, da $\frac{M}{a}>0$ ist. Bei positiver Schlüsselmasse und positiver Ausschüttungsquote erhält also mindestens eine Gemeinde Zuweisungen.

Für die strenge Monotonie auf dem Bereich $G \geqq \bar{K}_{1}$ bleibt $z u$ beweisen: $S$ ist an allen Intervallgrenzen $G=\bar{K}_{n}, n=1, \ldots, N$ stetig.

Dies ist erfüllt, denn wie man leicht zeigt, entsprechen die Funktionswerte

$$
s\left(\bar{K}_{n}\right)=\sum_{i=1}^{n-1} E_{i} b_{i}\left(\bar{k}_{n}-\bar{K}_{i}\right), n=1, \ldots, N
$$

den (rechtsseitigen) Grenzwerten:

$$
\lim _{\substack{h \rightarrow 0 \\ h>0}} s\left(\bar{K}_{n}+h\right)=s\left(\bar{K}_{n}\right), n=1, \ldots, N .
$$

$S$ ist also auf dem Bereich $\mathrm{G}>\overline{\mathrm{K}}_{1}$ streng monoton wachsend. Der Wertebereich ist $S(G) \in(0, \infty)$, somit existiert ein eindeutiges $G$, das der gesuchte Grundbetrag ist. 


\subsection{Iterationsverfahren für die Berechnung des Grund- betrags}

Aus dem Beweis der Existenz und Eindeutigkelt des Grundbetrags läßt sich ein Verfahren zu dessen praktischer Berechnung gewinnen. Die Schlüsselzuweisungen der Gemeinden können dann mit dem gefundenen Grundbetrag sehr leicht ermittelt werden, wenn auch die übrigen Daten vorliegen. In den Statistischen Landesämtern wird zur Berechnung des Grundbetrags allerdings ein anderes Verfahren verwendet, von dem wir nachweisen, daß es zum gesuchten Grundbetrag führt. Abschließend geben wir noch eine Modifikation dieses Verfahrens an, die etwas schneller sein dürte. Voraussetzung bei allen Verfahren ist wieder die ordnung der Gemeinden nach ihrer normierten steuerkraft.

\subsubsection{Verfahren (1)}

Das folgende Verfahren beruht auf der Idee, das Intervall $I_{n *}, n^{*} \in\{1, \ldots, N\}$ aus den Intervallen

$$
I_{n}= \begin{cases}\left(\bar{K}_{n}, \bar{K}_{n+1}\right], & n \in\{1, \ldots, N-1\} \\ \left(\bar{K}_{N}, \infty\right) & n=N\end{cases}
$$

zu bestimmen, in dem

$$
S(G)=\frac{M}{a}
$$

ist. Der Schnittpunkt ist offensichtlich im Intervall $I_{n} *$ falls für $n^{*} \in\{1, \ldots, N\}$ 
$(2.4 .1)$

$$
s\left(\bar{K}_{n *}\right)<\frac{M}{a} \leqq S\left(\bar{K}_{n *+1}\right), n^{*} \in\{1, \ldots, N-1\}
$$

$$
S\left(\bar{K}_{N}\right)<\frac{M}{a} \quad, n^{*}=N
$$

gilt.

Wegen der strengen Monotonie von $S$ kann man so vorgehen, daß man für $n=1, \ldots, N$ sukzessive prüft, ob

$$
S\left(\bar{K}_{n}\right)<\frac{M}{a}
$$

erfüllt ist. Falls für ein minimales $n^{*} \in\{1, \ldots, N-1\}$

$$
S\left(\bar{K}_{n *+1}\right) \geqq \frac{M}{a}
$$

gilt, d.h.

$$
\text { (2.4.2) } \quad n^{*}=\min \left\{n \mid S\left(\bar{K}_{n+1}\right) \geqq \frac{M}{a}\right\} \text {, }
$$

dann ist der Schnittpunkt zwischen $S(G)$ und $\frac{M}{a} 1 \mathrm{~m}$ Intervall $\left(\bar{K}_{n *}, \bar{K}_{n *+1}\right)$, und der Grundbetrag ergibt sich als

$$
G=\frac{\frac{M}{a}+\sum_{i=1}^{n^{*}} k_{i}}{\sum_{i=1}^{n^{*}} E_{i} b_{i}}
$$

Das Verfahren bricht ab. Ansonsten ist

$$
\text { (2.4.3) } \frac{M}{a}>S\left(\bar{K}_{N}\right) \text { und folglich } n^{*}=N
$$

mit dem Grundbetrag 


$$
G=\frac{\frac{M}{a}+\sum_{i=1}^{N} K_{i}}{\sum_{i=1}^{N} E_{i} b_{i}}
$$

\subsubsection{Das Verfahren der Statistischen Landes- ämter (2)}

Beim Verfahren der Statistischen Landesämter wird im Prinzip in jedem Schritt eine Annahme über die Zahl der zuweisungsberechtigten Gemeinden gemacht. Mit dieser Annahme läBt sich ein Grundbetrag berechnen, und mit diesem Grundbetrag wird überprüft, ob sich ein Widerspruch zur angenommenen Zahl der zuweisungsberechtigten Gemeinden ergibt. Dies wiederholt sich so lange, bis eine Annahme nicht zum Widerspruch geführt werden kann. ${ }^{1)}$

In der nun folgenden Darstellung bezeichnen wir die Schritte mit $j=0, \ldots, j^{*}$, die angenommene $\mathrm{Zahl}$ der zuweisungsberechtigten Gemeinden in schritt $j$ mit $n_{j}$ und den Grundbetrag bei $n_{j}$ Gemeinden mit $G\left(n_{j}\right)$.

\section{Algorithmus 2}

In einem ersten Schritt $(j=0)$ wird angenommen, alle Gemeinden seien zuweisungsberechtigt. Es gilt also

$$
n_{0}=N \text { und } G\left(n_{0}\right)=\frac{\frac{M}{a}+\sum_{i=1}^{N} K_{i}}{\sum_{i=1}^{N} E_{i} b_{i}} \text {. }
$$

1) Siehe auch die verbale Erläuterung des Verfahrens und ein fiktives Beispiel in Bohley, Foohs [1984], IV 10, Anhang zu Art. 1-6, S. 3-5. 
Es ist zu prüfen, ob die Annahme auch wahr ist, d.h. ob

(2.4.4) $G\left(n_{0}\right)>\bar{K}_{i}$ für $i=1, \ldots, N$

erfüllt ist. Ist dies der Fall, dann ist $G\left(n_{0}\right)$ der gesuchte Grundbetrag. Falls aber ein $n_{1} \in\{1, \ldots, N-1\}$ existiert mit

(2.4.5) $\quad G\left(n_{0}\right) \leqq \bar{k}_{i} \quad, \quad i=n_{1}+1, \ldots, N$,

dann führt die Annahme $\mathrm{n}_{0}=\mathrm{N}$ zum Widerspruch, und das Verfahren wird mit der Annahme $\mathrm{n}_{1}$ zuweisungsberechtigter Gemeinden (mit $n_{1}$ aus Bedingung (2.4.5)) und dem Grundbetrag

$$
G\left(n_{1}\right)=\frac{\frac{M}{a}+\sum_{i=1}^{n_{1}} k_{i}}{\sum_{i=1}^{n_{1}} E_{i} b_{i}}
$$

fortgesetzt. Falls

$$
G\left(n_{1}\right)>\bar{k}_{i} \quad \text { für } i=1, \ldots, n_{1},
$$

dann ist der Grundbetrag in $G\left(n_{1}\right)$ gefunden. Falls für ein $\mathrm{n}_{2}<\mathrm{n}_{1}$

$$
G\left(n_{1}\right) \leqq \bar{K}_{i} \quad, \quad i=n_{2}+1, \ldots, n_{1}
$$

gilt, dann ergibt sich ein Widerspruch, und das procedere wiederholt sich mit der Annahme $n_{2}, n_{3}$ usw., und zwar solange, bis in schritt $j=j^{*}$ ein $n_{j *}$ gefunden ist, für das
$(2.4 .6)$
$G\left(n_{j *}\right)>\bar{K}_{i}$
$i=1, \ldots, n_{j *}$

erfüllt ist.

Das Vorgehen beschreibt der 


\section{Algorithmus 2}

(O) Ausgangsdaten: $M, a, K_{i}, E_{i}, b_{i}, i=1, \ldots, N$.

(1) Initialisierung: $j:=0, n_{j}:=N$.

(2) Berechne: $G\left(n_{j}\right)=\frac{\frac{M}{a}+\sum_{i=1}^{n_{j}} k_{i}}{\sum_{i=1}^{n} E_{i} b_{i}}$.

Falls $G\left(n_{j}\right)>\bar{K}_{i}$ für $i=1, \ldots, n_{j}$, dann gehe $z u(3)$. Berechne ein $n_{j+1}<n_{j}$ mit

$$
G\left(n_{j}\right) \leqq \bar{K}_{i} \quad, \quad i=n_{j+1}+1, \ldots, n_{j} .
$$

Setze $j:=j+1$.

Gehe zu (2).

(3) Stop. Grundbetrag gefunden.

Wir wollen nun beweisen, daß mit dieser Vorgehensweise auch tatsächlich der "richtige" Grundbetrag, d.h. die Lösung von (2.1.10) gefunden wird. Das ist keineswegs selbstverständlich, denn das Verfahren geht implizit von der (unbewiesenen) Annahme aus, daB die sukzessiv ermittelten Grundbeträge zumindest nicht gröBer werden.

Denn würde für $j \geqq 1$ und $n_{j}<n_{j-1}$

$$
G\left(n_{j}\right)>G\left(n_{j-1}\right)
$$

sein, dann wäre immer 


$$
G\left(n_{j}\right)>\bar{K}_{i} \quad, \quad i=1, \ldots, n_{j}
$$

erfüllt, und das Verfahren würde abbrechen, obwohl es Gemeinden $i>n_{j}$ geben kann, für die

$$
G\left(n_{j}\right)>\bar{K}_{i}
$$

gilt, d.h. die Annahme, beim Grundbetrag $G\left(n_{j}\right)$ erhalten genau $n_{j}$ Gemeinden Zuweisungen, falsch wäre.

Es ist also für $\mathrm{j} \geqq 1$ und $n_{j}<n_{j-1}$ zu zeigen :

$(2.4 .7) \quad G\left(n_{j}\right) \leqq G\left(n_{j-1}\right)$.

Mit einigen Umformungen erhält man die Bedingung

$$
G\left(n_{j}\right) \leqq G\left(n_{j-1}\right)
$$

$(2.4 \cdot 7 \mathrm{a})$

$$
\sum_{i=n_{j}+1}^{n_{j-1}} E_{i} b_{i} G\left(n_{j-1}\right) \leqq \sum_{i=n_{j}+1}^{n_{j-1}} K_{i} .
$$

Sie ist immer erfüllt, da die Uberprüfung der Annahme in schritt $j$ bekanntlich

$$
G\left(n_{j-1}\right) \leqq \bar{K}_{i} \quad, \quad i=n_{j}+1, \ldots, n_{j-1}
$$

ergeben hatte und folglich

$$
E_{i} b_{i} G\left(n_{j-1}\right) \leqq k_{i} \quad, i=n_{j}+1, \ldots, n_{j-1}
$$

gilt. Daraus folgt (2.4.7a). 


\subsubsection{Ein modifiziertes Verfahren (3)}

Man kann sich leicht ein Verfahren überlegen, bei dem in einem ersten Schritt $(j=0)$ von der Annahme ausgegangen wird, daB genau $n_{0}$ Gemeinden Zuweisungen erhalten. Dabei ist $n_{0} \in\{1, \ldots, N\}$ eine beliebige Gemeinde, zweckmäßigerweise wird man $n_{0}=\frac{N}{2}$ wählen ( $N$ gerade), wenn man davon ausgeht, daß die Wahrscheinlichkeit, daß genau n Gemeinden Zuweisungen erhalten, für alle $n \in\{1, \ldots, N\}$ gleichverteilt ist. Man könnte auch die Zahl der Gemeinden nehmen, die im Vorjahr Zuweisungen erhielten, wenn sich die Daten aus dem Vorjahr nicht sehr verändert haben.

Bei dem Verfahren kommt es darauf an, in einem ersten Schritt festzustellen, ob die angenommene $\mathrm{Zahl} \mathrm{n}_{\mathrm{o}}$ der Gemeinden $z u$ klein oder $z u$ groß ist. Beim Verfahren der Statistischen Landesämter ist dies nicht nötig, da $\mathrm{N}$ immer $z u$ groB ist, wenn $G(N)$ nicht die Lösung war. Falls $n_{0} z u$ groß ist, dann läuft das Verfahren analog zu Algorithmus 2 weiter, es kommt u.U. allerdings mit weniger schritten aus, weil man es a priori mit weniger Gemeinden begonnen hat. Falls $n_{0}$ kleiner als die gesuchte $\mathrm{Zahl}$ ist, dann kann man zeigen, daß die gesuchte $\mathrm{zahl}$ der Gemeinden $\mathrm{n}_{j^{*}}$ in jedem Falle in dem Bereich

$$
n_{0}<n_{j *} \leqq n_{1}
$$

liegt, wobei $n_{1}$ die $\mathrm{zahl}$ der Gemeinden $i$ ist, für die

$$
G\left(n_{0}\right)>\bar{K}_{i}
$$

erfüllt ist. Da $n_{j^{*}} \leqq n_{1}$ ist, läuft das Verfahren dann wieder analog zu Algorithmus 2 ab.

Beschreiben wir die Vorgehensweise etwas genauer: 


\section{Algorithmus 3}

(1) $j=0$ : Annahme: $n_{0} \in\{1, \ldots, N\}$ beliebig.

(2) Falls

$$
G\left(n_{0}\right) \begin{cases}\leqq \bar{k}_{i}, & i=n_{0}+1, \ldots, N \\ >\bar{k}_{i}, & i=1, \ldots, n_{0},\end{cases}
$$

dann ist $G\left(n_{0}\right)$ der gesuchte Grundbetrag, und das Verfahren bricht ab.

(3) Falls für ein $n_{1}<n_{0}$

$$
G\left(n_{0}\right) \leqq \bar{K}_{i} \quad, \quad i=n_{1}+1, \ldots, N
$$

gilt, dann ist wegen $G\left(n_{0}\right) \geqq G\left(n_{1}\right)$ (siehe $(2.4 .7)$ ) $n_{0}$ $z u$ groß, und das Verfahren wird wie in Algorithmus 2 mit Schritt $j=1$ und der Annahme $n_{1}$ aus (2.4.9) fortgesetzt.

(4) Falls für ein $\mathrm{n}_{1}>\mathrm{n}_{\mathrm{o}}$

$$
G\left(n_{0}\right)>\bar{K}_{i} \quad, \quad i=1, \ldots, n_{1}
$$

gilt, dann ist $n_{0}$ zu klein, und das Verfahren wird mit $n_{1}$ aus (2.4.10) und $G\left(n_{1}\right)$ fortgesetzt. Da unter der Voraussetzung (2.4.10) diesmal statt (2.4.7)

$$
G\left(n_{1}\right)<G\left(n_{0}\right) \quad \text { für } n_{1}>n_{0}
$$

gilt (Beweis analog zum Beweis von (2.4.7)), kann die gesuchte $Z$ ahl der Gemeinden $n_{j *}$ wegen $(2.4 .10)$ nicht größer als $n_{1}$ sein, d.h. es gilt

$$
\mathrm{n}_{0}<\mathrm{n}_{j^{*}} \leqq \mathrm{n}_{1}
$$


Folglich existiert ein $\mathrm{n}_{2} \leqq \mathrm{n}_{1}$ mit

$$
G\left(n_{1}\right)>\bar{K}_{i}, i=1, \ldots, n_{2} .
$$

Falls $\mathrm{n}_{2}=\mathrm{n}_{1}$ ist, dann ist $\mathrm{G}\left(\mathrm{n}_{1}\right)$ der gesuchte Grundbetrag. Falls $n_{2}<n_{1}$ ist, dann wird das Verfahren analog zu Algorithmus 2 mit der Annahme $n_{2}$ und $G\left(n_{2}\right)$ fortgesetzt.

口

Das Verfahren der Statistischen Landesämter ist also keineswegs das einzige, mit dem man die Schlüsselzuweisungen berechnen könnte. Wir wollen aber nicht näher darauf eingehen, welches dieser Verfahren etwa schneller oder einfacher ist und welches Verwendung finden sollte. Daß aber überhaupt ein Iterationsverfahren notwendig ist, macht die Unhandlichkeit und Intransparenz der Schlüsselzuweisungen deutlich. So dürfte kaum eine Gemeinde in der Lage sein, die Richtigkeit der ihr zugewiesenen Mittel zu überprüfen. Sie kann vermutlich auch nicht einschätzen, ob sie bei einer anderen Schlüsselmasse oder einem anderen Ausgleichssatz besser oder schlechter gestellt wäre. Denn die Statistischen Landesämter ermitteln nur die Schlüsselzuweisungen, wie sie sich in einem bestimmten Jahr aus der tatsächlich verfügbaren Schlüsselmasse und dem gerade gesetzlich fixierten Ausgleichssatz ergeben. Zwar könnte man sich durch den wiederholten Einsatz eines Iterationsverfahrens zu der tatsächlichen Verteilung der Schlüsselzuweisungen eines bestimmten Jahres auch alternative Verteilungen beschaffen, allerdings nicht mit der nötigen Beliebigkeit, denn sämtliche exogenen Größen müssen jeweils spezifiziert sein. Dies reicht für eine formale Analyse der Schlüsselzuweisungen aber noch nicht aus. Es ist dazu notwendig, alternative Verteilungen nicht empirisch durch die Anwendung von Iterationsverfahren $z$ erzeugen, sondern durch eine beliebige 
Variation exogener Größen, die man als Parameter auffaßt. Dazu müBte es gelingen, den Grundbetrag als Funktion interessierender Parameter anzugeben, mit der man sich beliebig viele Verteilungen der Schlusselzuweisungen auf analytischem Wege beschaffen kann. Wir werden uns mit dieser Problemstellung in Teil II noch ausführlich beschäftigen. Zuvor wollen wir jedoch noch einige Kriterien entwickeln, mit denen Verteilungen aus staatlicher und kommunaler sicht beurteilt werden können. 
3 Verteilungsziele und Verteilungskriterien

Die Schlüsselzuweisungen $S_{1} \ldots, S_{N}$ an die Gemeinden $1, \ldots, N$ sind, wie wir erkannt haben, eindeutig bestimmt, wenn die exogenen Größen spezifiziert werden. Den Vektor $s=\left(s_{1}, \ldots, s_{N}\right)$ nennen wir Verteilung der Schlüsselzuweisungen. Wir interessieren uns nun für die Frage, wie eine Verteilung aus staatlicher und kommunaler sicht $z u$ beurteilen ist. Dazu benötigen wir Kriterien, und bei ihrer Spezifikation orientieren wir uns an den Zielen, die staat und Kommunen mit Schlüsselzuwelsungen verfolgen. Meist kann eine Verteilung nur durch einen Vergleich mit alternativen Verteilungen beurteilt werden. Deshalb gehen wir in diesem Kapitel davon aus, das man sich durch Variation von Parametern, etwa der Schlüsselmasse oder der Ausschuttungsquote, beliebige Verteilungen beschaffen kann. Die Menge aller alternativen Verteilungen bezeichnen wir nachfolgend mit $A$.

\section{1 Kommunale Ziele}

Wir gehen im folgenden von elgennutzorientiertem Verhalten der Kommunen aus, und zwar nehmen wir an, daß sie über möglichst hohe finanzielle Mittel verfügen möchten. Nimmt man die Steuerkraft und die sonstigen Einnahmemöglichkeiten als gegeben an, so sind die Kommunen also an möglichst hohen Zuweisungen interessiert.

Diese Verhaltensannahmeerscheint plausibel. Aus der Sicht einer Gemeinde ist es rational, das Angebot an öffentlichen Gütern auszudehnen, wenn diese Güter mit Zuweisungen finanziert werden können. Im kommunalen Budget entsteht ein Entlastungseffekt, der die Finanzierung weiterer Ausgaben ermöglicht. Die Grenzkosten der zusätzlichen öffentlichen Güter sind subjektiv gesehen gleich Null, man kann hier auch von 
einer Finanzierungsillusion ${ }^{1)}$ sprechen. Zuweisungen könnten natürlich auch dazu benutzt werden, die Steuerbelastung der Bürger zu reduzieren ${ }^{2}$, was die Attraktivität einer Kommune als Wohn- und Standort erhöhen würde.

Zur Präzisierung des kommunalen ziels benötigen wir zunächst zwei Definitionen:

Sei $s=\left(s_{1}, \ldots, s_{N}\right) \in A$ eine beliebige Verteilung der schlüsselzuweisungen. Die Finanzausstattung $\mathrm{F}_{\mathrm{i}}(\mathrm{s})$ einer Gemeinde $\mathrm{i}$ bei der Verteilung $s$ ist definiert als

(3.1.1) $\quad F_{i}(s):=s_{i}+K_{i}$.

Sie gibt an, über wieviele finanzielle Mittel eine Gemeinde verfügt. Um den Einfluß verschiedener Verteilungen auf die Finanzausstattung beurteilen zu können, ist dabei angenommen, daß sich die Finanzausstattung nur aus den (fixen) normalisierten steuereinnahmen $K_{i}$ und eben der Schlüsselzuweisung zusammensetzt. Von Gebühren, sonstigen Zuweisungen und Einnahmen, die über die normalisierte steuerkraft hinausgehen, wird abstrahiert.

Der Finanzanteil $A_{i}$ (s) einer Gemeinde $i$ bei $s$ ist definiert als

$$
\text { (3.1.2) } \quad A_{i}(s):=\frac{F_{i}(s)}{\sum_{j=1}^{N} F_{j}(s)}
$$

und gibt den Anteil der Finanzausstattung der Gemeinde $i$ an der Summe aller Finanzausstattungen an.

1) Zimmermann, Henke [1982], S. 36 .

2) Zimmermann [1983], S. 45. 
Für eine Gemeinde kommen unter der genannten zielsetzung überhaupt nur Verteilungen $s \in A$ mit der Eigenschaft

$$
\text { (3.1.3) } \quad F_{i}(s)>K_{i}
$$

bzw. der damit äquivalenten Eigenschaft

$$
(3.1 .4) \quad s_{i}>0
$$

in Betracht. Die Steuereinnahmen sollen also mit Schlüsselzuweisungen aufgestockt werden. Damit verbessert eine Gemeinde zwar ihre absolute Position, aber die Eigenschaft (3.1.3) sagt nichts über ihre relative Position aus. Bezeichne

$$
F(s):=\sum_{i=1}^{N} F_{i}(s) \text { und } K:=\sum_{i=1}^{N} K_{i}
$$

dann ist bei einer positiven Schlüsselmasse stets

$$
(3.1 .5) \quad F(s)>K \text {, }
$$

und es können Gemeinden absolut besser gestellt werden, ohne daß gleichzeitig andere Gemeinden (absolut) schlechter gestellt werden müssen, d.h. ohne daß

$$
F_{j}(s)<K_{j} \quad \text { für mindestens ein } j \in\{1, \ldots, N\}
$$

ist. Eine Gemeinde, die ihre relative Position verbessern will, wird von einer Verteilung statt (3.1.3) die Eigenschaft

$$
(3.1 .6) \quad A_{i}(s)>\frac{K_{i}}{K}
$$

fordern. Dabei ist $K_{i} / K$ der steuerkraftanteil der Ge- 
meinde i. Da für alle $s \in A$

$$
\sum_{i=1}^{N} A_{i}(s)=\sum_{i=1}^{N} \frac{K_{i}}{K}=1
$$

gilt, ist der Finanzanteil von $i$ nur dann höher als ihr Steuerkraftanteil, wenn gleichzeitig in mindestens einer anderen Gemeinde der Finanzanteil geringer als der Steuerkraftanteil ist. Dann wird die Gemeinde $i$ auch relativ besser gestellt und dafür muß mindestens eine andere schlechter gestellt werden.

Die Eigenschaften (3.1.3) und (3.1.6) sind als Minimalforderung anzusehen, und es gibt sicher viele Verteilungen, die sie erfüllen. Eine Gemeinde kann die Alternativen aber leicht in eine Rangordnung bringen:

(3.1.7) Seien $\hat{s}, s \in A$ irgendzwei alternative Verteilungen.

$\hat{\mathbf{s}}$ ist für die Gemeinde $i$ günstiger als $\mathbf{s}$

- $F_{i}(\hat{s})>F_{i}(s)$.

Die Finanzausstattung einer Gemeinde $i$ ist bei jeder Verteilung eindeutig bestimmt, und somit können alle Alternativen miteinander verglichen werden. Es existieren deshalb auch optimale Verteilungen, die die Gemeinde allen anderen vorzieht. Das sind unter der genannten zielsetzung die Verteilungen mit maximaler Finanzausstattung:

(3.1.8) Eine Verteilung $\mathbf{s}_{i}^{*} \in A$ ist optimal für die Gemeinde $i$

$$
-F_{i}\left(s_{i}^{*}\right)=\max _{s \in A} F_{i}(s) .
$$


Falls $F(s) \neq F(\hat{s})$ für irgendzwei $s, \hat{s} \in A$ ist, dann ist die relative Position einer Gemeinde $i$ bei $\hat{s}$ besser als bei $s$

$$
(3.1 .9) \leftrightarrow A_{i}(\hat{s})>A_{i}(s),
$$

und sie ist optimal bei einem $\mathbf{s}_{i}^{*} \in A$

$$
(3.1 .10) \rightarrow A_{i}\left(s_{i}^{*}\right)=\max _{s \in A} A_{i}(s) .
$$

Ein maximaler Finanzanteil der Gemeinde $i$ bei $\mathbf{s}_{1}^{*}$ bedeutet, daß gleichzeitig der gesamte Finanzanteil aller ubrigen Gemeinden minimal, also niedriger ist als bei jeder anderen (für $i$ suboptimalen) Verteilung. Man kann sich daher leicht vorstellen, daß sich die optimalen Verteilungen der Gemeinden voneinander unterscheiden.

In jedem Jahr wird nur eine Verteilung realisiert, eben diejenige, die sich aus den gesetzlichen Bestimmungen ergibt. Sie soll in erster Linie staatlichen Zielen (die wir gleich präzisieren) dienen. Insofern haben die Gemeinden überhaupt keine Möglichkeit, die für sie günstigste Verteilung auszuwählen. Wir wollen auch gar nicht behaupten, daß die von uns betrachteten Parameter (die Schlüsselmasse und die Ausschüttungsquote) von den Gemeinden als Instrumentvariablen benutzt werden können. Uns kommt es aus kommunaler sicht in dieser Untersuchung vielmehr darauf an, die optimalen Verteilungen der Gemeinden mit der Verteilung zu vergleichen, die vom staat präferiert wird. Damit können wir auch angeben, welche Gemeinden bei dieser Verteilung sozusagen von ihrem optimalen zustand weit entfernt sind und welche diesem sehr nahe kommen. 


\subsection{Staatliche Ziele}

In der Literatur werden im wesentlichen drei Funktionen der Schlüsselzuweisungen genannt, ${ }^{1)}$ die man als staatliche ziele ansehen kann:

1) Fiskalziel

Aufstockung der kommunalen Finanzmasse.

2) Redistribution

Gleiche Unterschiede zwischen Finanzbedarf und Steuerkraft unter den Gemeinden. 2)

3) Raumordnungsziel

Finanzieller Ausgleich für zentralörtliche Aufgaben.

Bei der Präzisierung dieser ziele werden wir sehen, daß sie sich nicht exakt voneinander trennen und im Grunde auf Vorstellungen zurückführen lassen, die sich schon bei Popitz ${ }^{3)}$ und im PreuBischen Finanzausgleichsgesetz finden (siehe Einleitung).

Bei der fiskalischen Zielsetzung wird davon ausgegangen, daß die eigenen Steuereinnahmen nicht in allen Gemeinden zur Deckung des Finanzbedarfs ausreichen und durch Zuweisungen aufgestockt werden müssen. Man könnte diese Vorstellung etwa so präzisieren:

1) Vgl. zuletzt: Hoppe [1985], S. 88; siehe auch: Weber [1981], S. 56 ff.; Münstermann [1975], S. 140. Eine vollständige Auflistung der Ziele des kommunalen Finanzausgleichs findet sich etwa in: Smekal [1980]; Gläser [1981], S. $48 \mathrm{ff}$.; Seiler [1980], S. $15 \mathrm{ff}$.

2) Siehe Musgrave [1961], S. 102: "Equalization of Differentials in Need and Capacity" (Plan 3).

3) Popitz [1932]. 


\section{(3.2.1) Fiskalziel (1)}

Für eine Verteilung $\mathbf{s \in A}$ soll gelten:

$$
\begin{array}{ll}
F_{i}(s) \geqq K_{i}, & i=1, \ldots, N \text { und } \\
F_{i}(s)>K_{i} & \text { für mindestens ein } i \in\{1, \ldots, N\} .
\end{array}
$$

Dies besagt: Bei einer Verteilung sollte die Finanzausstattung in keiner Gemeinde geringer als die steuerkraft dieser Gemeinde sein, in mindestens einer Gemeinde aber (echt) gröBer. Diese scheinbar recht harmlose Forderung schränkt die redistributiven Möglichkeiten stark ein. Sie kann nur dann erfüllt werden, wenn die finanziellen Mittel, mit denen die Steuereinnahmen mindestens einer Gemeinde aufgestockt werden sollen, nicht von den Gemeinden (über Umlagen) selbst aufgebracht werden müssen. Betrachten wir statt der Schlüsselzuweisungen die Zuweisungen $\mathrm{z}_{1}, \ldots, \mathrm{z}_{\mathrm{N}^{\prime}}$ von denen wir nur

$$
\sum_{i=1}^{N} z_{i}=0 \text { und } z_{i} \neq 0 \text { für mindestens ein } i \in\{1, \ldots, N\}
$$

fordern. Dann ist (3.2.1) nie erfüllt, weil es für eine beliebige Verteilung $z=\left(z_{1}, \ldots, z_{N}\right)$ kein $i \in\{1, \ldots, N\}$ mit $F_{i}(z)>K_{i}$ gibt, ohne daß $F_{j}(z)<K_{j}$ und somit $z_{j}<0$ ist (Umlage) für mindestens ein $j \in\{1, \ldots, N\}$ mit $j \neq i$.

Wir werden sehen (siehe 5.1.1), daß bei den Schlüsselzuweisungen (3.2.1) immer erfültt ist. Denn bei einer positiven Schlüsselmasse gibt es in jeder Verteilung $\mathbf{s}$ mindestens eine Gemeinde $i \in\{1, \ldots, N\}$ mit $s_{i}>0$, und gleichzeitig gibt es keine Gemeinde $j \in\{1, \ldots, N\}, j \neq i$ mit $F_{j}(s)<k_{j}$.

Verteilungen, die (3.2.1) genügen, beinhalten quasi eine Besitzstandsgarantie: Keine Gemeinde muB von ihren eigenen Einnahmen etwas abgeben, um die Zuweisungen an andere Ge- 
meinden zu finanzieren. Dies bedeutet aber auch, daß redistributive Ziele (die wir glelch präzisieren) überhaupt nicht mehr $z$ u realisieren sind, falls nicht finanzielle Mittel vom Land zur Verfügung gestellt werden. Zwar ist über ein System von Zuweisungen, bei dem vermeintlich niemand schlechter gestellt wird, ${ }^{1)}$ eher ein Konsens unter den Gemeinden herzustellen, das Land könnte sich aber für seine redistributiven ziele einen immer größeren Teil der Steuereinnahmen aneignen, die hoheitlich den Gemeinden zustehen. Bei den Schlüsselzuweisungen ist dies umso eher zu befürchten, als die Verteilung mit steigender Schlüsselmasse progressiver wird (siehe 5.4).

Eine Abschwächung von (3.2.1) ist die Bedingung

(3.2.2) Fiskalziel (2)

$$
F_{1}(s)>K_{i} \text { für mindestens ein } i \in\{1, \ldots, N\}, s \in A
$$

Diese Forderung ist nicht sehr selektiv, denn sie wird, wie man sich leicht überlegt, sogar von einer beliebigen Verteilung der zuweisungen $z_{1} \ldots, z_{N}$ (und erst recht von den Schlüsselzuweisungen) erfüllt. Es wird auch nicht präzisiert (ebenso nicht in (3.2.1)), bei welchen Gemeinden die Steuereinnahmen aufgestockt werden sollten. Man sieht sich also sofort vor die Frage gestellt, wie eine Finanzmasse zu verteilen ist.

Ein Maß für die Bedürftigkeit einer Gemeinde im Vergleich zu irgendeiner anderen Gemeinde ist die Differenz zwischen ihrem normierten Finanzbedarf und ihrer normierten steuerkraft

$$
\bar{B}_{i}(s)-\bar{K}_{i}, \quad s \in A
$$

1) Erinnern wir uns, relativ gesehen wird natürlich mindestens eine Gemeinde schlechter gestellt (siehe 3.1). 
Eine Gemeinde $j$ nennen wir nicht ärmer als eine Gemeinde i, wenn

$$
\text { (3.2.3) } \quad \bar{B}_{i}(s)-\bar{K}_{i} \geq \bar{B}_{j}(s)-\bar{K}_{j}, \quad i, j \in\{1, \ldots, N\}
$$

ist. Dabei wird unter dem Finanzbedarf einer Gemeinde der Finanzbedarf verstanden, wie er für die Schlüsselzuweisungen definiert ist. Da er bekanntlich vom Grundbetrag abhängt, ist er auch von einer bestimmten Verteilung s abhängig, weil zu jedem Grundbetrag eine bestimmte Verteilung der Schlüsselzuweisungen gehört. Da

(3.2.4) $\quad \bar{B}_{i}(s)=G(s), i=1, \ldots, N$,

gilt, wobei $G(s)$ der Grundbetrag bei der Verteilung $s$ ist, ist (3.2.3) äquivalent $\mathrm{zu}$

$$
\text { (3.2.5) } \quad \overline{\mathrm{K}}_{i} \leqq \overline{\mathrm{K}}_{j}, i, j \in\{1, \ldots, \mathrm{N}\} \text {. }
$$

Die Armut einer Gemeinde $i$ im Vergleich zu einer Gemeinde j hängt also nicht, wie befürchtet, von einer bestimmten Verteilung ab. Die Ordnung der Gemeinden nach ihrer normierten Steuerkraft ist daher gleichzeitig auch eine Rangordnung der Armut, und sie kann zudem als eine Reihenfolge der Bedürftigkeit für die Verteilung der Schlüsselzuweisungen interpretiert werden. Ob eine bedürftige Gemeinde durch eine Verteilung $s$ besser gestellt wird, läßt sich leicht an ihrem Finanzierungsdefizit bei $s$ ablesen.

Das Finanzierungsdefizit $D_{i}(s)$ einer Gemeinde $i$ bei der Verteilung $\mathbf{s}$ ist durch

$(3.2 .6) \quad D_{i}(s):=B_{i}(s)-F_{i}(s), s \in A$

definiert. Es gibt also den finanziellen Betrag an, der einer Gemeinde trotz Schlüsselzuweisungen zur Deckung des 
Finanzbedarfs noch fehlt. Falls $D_{i}(s)<0$ ist, dann hat die Gemeinde einen FinanzierungsüberschuB, d.h. ihre finanziellen Mittel übersteigen den Bedarf. Für einen Vergleich der Finanzierungsdefizite verschiedener Gemeinden benötigen wir das normierte Finanzierungsdefizit einer Gemeinde $i$ bei $\mathbf{s}$

(3.2.7) $\quad \bar{D}_{i}(s):=\frac{D_{i}(s)}{E_{i} b_{i}}, \quad i=1, \ldots, N, s \in A$,

bei dem $D_{i}(s)$ wieder auf einen repräsentativen Einwohner von $i$ bezogen ist.

Mit der normierten Finanzausstattung $\bar{F}_{i}:=F_{i} / E_{i} b_{i}$ erhält man

(3.2.8) $\quad \bar{D}_{i}(s)=G(s)-\bar{F}_{i}(s)$ für $i=1, \ldots, N, s \in A$.

Die Abhängigkeit der normierten Finanzierungsdefizite der Gemeinden von einer Verteilung $s \in A$ ist hier durchaus sinnvoll, denn wir wollen Unterschiede in den normierten Finanzierungsdefiziten der Gemeinden als Maß für die redistributive Wirkung einer Verteilung nehmen. Wie man schon an (3.2.8) sieht, wird dabei die Verteilung der normierten Finanzausstattungen eine entscheidende Rolle spielen, weil diese mit einer für alle Gemeinden gleichen Größe, dem normierten Finanzbedarf, verglichen werden.

Eine Maximalforderung an eine Verteilung $s \in A$ wäre sicher

(3.2.9) Fiskalische Gleichheit (1)

$$
\bar{D}_{i}(s)=0 \quad \text { für alle } i=1, \ldots, N \text {. }
$$

Eine Verteilung $s$ hat also die Eigenschaft (3.2.9), wenn in keiner Gemeinde ein Finanzierungsüberschuß bzw. Finanzierungsdefizit verbleibt. Dies ist genau dann der Fall, wenn in allen Gemeinden die normierte Finanzausstattung 
gleichverteilt ist und zusätzlich noch genau so groß ist wie der Grundbetrag bei $s$, denn es gilt für alle $i=1, \ldots, N$ :

$$
\bar{D}_{i}(s)=0 \Leftrightarrow \bar{F}_{i}(s)=G(s)
$$

Dies ist eine sehr restriktive Forderung, die man noch abschwächen kann:

\section{(3.2.10) Fiskalische Gleichheit (2)}

$$
\bar{D}_{i}(s)=\bar{D}_{j}(s), i, j \in\{1, \ldots, N\}
$$

Es wird hier nur noch verlangt, daB die normierten Finanzierungsdefizite in allen Gemeinden gleich groß sind, was wegen

$$
\bar{D}_{i}(s)=\bar{D}_{j}(s) \cdot \bar{F}_{i}(s)=\bar{F}_{j}(s), i, j \in\{1, \ldots, N\}
$$

bei Gleichverteilung der normierten Finanzausstattungen immer gegeben ist. Es sind also auch Finanzierungsdefizite (bzw. Finanzierungsüberschüsse) zugelassen, sie müssen allerdings, bezogen auf einen repräsentativen Einwohner, in allen Gemeinden gleich sein.

Wenn man fiskalische Gleichheit ablehnt, ${ }^{1)}$ wird man zumindest die Progressivität einer Verteilung fordern. Uber die Progressivität einer Verteilung kann nur im Vergleich mit einer anderen Verteilung eine Aussage gemacht werden:

\section{(3.2.11) Progressivität}

Seien $s, \hat{s} \in A$ zwei Verteilungen der Schlüsselzuweisungen. Seien $x=\left(\bar{F}_{1}(s), \ldots, \bar{F}_{N}(s)\right)$ und

1) Vgl. zuletzt: Thiem [1984], S. 178 . 
$\mathrm{Y}=\left(\overline{\mathrm{F}}_{1}(\hat{\mathrm{s}}), \ldots, \overline{\mathrm{F}}_{\mathrm{N}}(\hat{\mathrm{S}})\right)$ die zugehörigen Verteilungen der Finanzausstattungen bei den Alternativen $\mathrm{s}$ und $\hat{\mathrm{s}}$.

Dann ist $\mathbf{s}$ progressiver als $\hat{s} \rightarrow x$ ist"gleicher" als $y$.

Die Alternativen sind natürlich im jeweiligen Fall zu konkretisieren. Es können etwa die Verteilungen bei verschiedenen Ausschüttungsquoten (siehe 6.4) oder bei verschiedenen Schlüsselmassen (siehe 5.4 ) sein. Dann besagt also (3.2.11), daß von zwei Verteilungen diejenige progressiver ist, bei der die Verteilung der normierten Finanzausstattungen gleicher ist. Es wird aber überhaupt nicht präzisiert, was unter "Gleichheit"bzw. "Ungleichheit "einer Verteilung $\mathrm{zu}$ verstehen ist und wie sie gemessen werden sollte. Wir werden dazu das Lorenzkriterium benutzen und darauf im nächsten Abschnitt ausführlich eingehen.

Eine Minimalforderung an eine Verteilung $\mathbf{s}$ ist wohl die

\section{(3.1.12) Nichtregressivität}

Sei s eine Verteilung der Schlüsselzuweisungen und $x=\left(\bar{F}_{1}(s), \ldots, \bar{F}_{N}(s)\right)$ die zugehörige Verteilung der normierten Finanzausstattungen. Sei $t=\left(\bar{K}_{1}, \ldots, \bar{K}_{N}\right)$ die Verteilung der normierten Steuerkraft.

s ist nicht regressiv $\Leftrightarrow \mathrm{x}$ ist mindestens so gleich wie $t$.

Hier wird von einer Verteilung s also verlangt, die von ihr erzeugte Verteilung der normierten Finanzausstattungen soll zumindest nicht ungleicher sein als die Verteilung der normierten Steuerkraft. (Wann eine Verteilung mindestens so gleich wie eine andere Verteilung ist, wird in 3.3 präzisiert.) 
In der Literatur wird das redistributive Ziel manchmal auch so verstanden, daß die Finanzausstattung pro Einwohner $z u$ nivellieren ist. ${ }^{1)}$ will man damit die Finanzierungsdefizite pro Einwohner in den Gemeinden ausgleichen, dann setzt dies einen in allen Gemeinden gleichen Finanzbedarf pro Einwohner voraus. Die Annahme eines identischen Pro-Kopf-Finanzbedarfs ist aber allein schon wegen der zentralörtlichen Funktionen bestimmter Kommunen nicht sinnvoll (Raumordnungsziel). Auch in Kommunen der gleichen zentralitätsstufe ist der Bedarf pro Einwohner praktisch immer verschieden, wenn Bedarf mit einem Bedarfsindex gemessen wird, der sich aus mehreren Bedarfsindikatoren zusammensetzt. ${ }^{2}$ ) Geht man also von einem unterschiedlichen Finanzbedarf pro Einwohner aus, dann ist bei Gleichverteilung der Finanzausstattung pro Einwohner das Finanzierungsdefizit pro Einwohner verschieden, was der ursprünglichen Absicht zuwiderläuft. Die Nivellierung der normierten Finanzausstattungen ist eine schwächere Forderung. Sie läßt einen unterschiedlichen Pro-Kopf-Finanzbedarf zu. Man kann in den Bedarfsindexwerten dann auch Raumordnungsziele berücksichtigen, worauf wir in dieser Arbeit aber nicht näher eingehen.

\subsection{Kriterien der fiskalischen Ungleichheit}

Um die Progressivität $(3.2 .11)$ bzw. Nichtregressivität (3.2.12) einer Verteilung der Schlüsselzuweisungen zu präzisieren, führen wir das Lorenzkriterium ein.

Dazu sei $\mathbf{s} \in \mathbf{A}$ eine beliebige Verteilung der Schlüsselzuweisungen.

1) Vgl. Münstermann [1975], S. 140.

2) Vgl. Hanusch, Kuhn [1985]. 
Den Vektor

$$
\text { (3.3.1) } \left.\quad\left(\overline{\mathrm{F}}_{1}(\mathrm{~s}), \ldots, \overline{\mathrm{F}}_{\mathrm{N}}(\mathrm{s})\right) \in \mathbb{R}_{++}^{N} 1\right)
$$

nennen wir die Verteilung der normierten Finanzausstattungen bei $\mathbf{s}$. Da für zwei Verteilungen $s, \hat{s} \in A$ die Summen

$$
\sum_{i=1}^{N} \bar{F}_{i}(s) \text { und } \sum_{i=1}^{N} \bar{F}_{i}(\hat{s})
$$

(auch bei gleicher Schlüsselmasse) praktisch immer verschieden sind, müssen wir zur Beurteilung der Progressivität bzw. Nichtregressivität einer Verteilung auf die normierten Finanzanteile rekurrieren.

Der normierte Finanzanteil $\bar{A}_{i}(s)$ einer Gemeinde $i$ bei $s \in A$ ist definiert als Anteil der normierten Finanzausstattung von $i$ an der summe aller normierten Finanzausstattungen bei $\mathbf{s}$

$$
\text { (3.3.2) } \quad \overline{\mathrm{A}}_{i}(s):=\frac{\overline{\mathrm{F}}_{i}(s)}{\sum_{j=1}^{N} \bar{F}_{j}(s)} .
$$

Man sieht, es gilt

$$
\sum_{i=1}^{N} \bar{A}_{i}(s)=1 \quad \text { für alle } s \in A
$$

Den Vektor

(3.3.3) $\quad\left(\overline{\mathrm{A}}_{1}(\mathrm{~s}), \ldots, \overline{\mathrm{A}}_{\mathrm{N}}(\mathrm{s})\right) \in \mathbf{R}_{++}^{\mathrm{N}}, \mathrm{s} \in \mathrm{A}$ 
nennt man die relative Verteilung der normierten Finanzausstattungen bzw. die Verteilung der normierten Finanzanteile bei $\mathbf{s}$.

Eine Verteilung $\left(\overline{\mathrm{A}}_{1}(\mathrm{~s}), \ldots, \overline{\mathrm{A}}_{N}(\mathrm{~s})\right)$ heiBt geordnet, falls

$$
\bar{A}_{1}(s) \leqq \bar{A}_{2}(s) \leqq \cdots \leqq \bar{A}_{N}(s)
$$

gilt. Dies ist für alle sEA genau dann der Fall, wenn

$$
(3.3 .4) \quad \bar{F}_{1}(s) \leqq \bar{F}_{2}(s) \leqq \cdots \leqq \bar{F}_{N}(s)
$$

gilt, d.h. die Gemeinden in der Reihenfolge ihrer normierten Finanzausstattung numeriert sind.

Der Einfachheit halber schreiben wir nachfolgend für eine Verteilung $\left(\bar{A}_{1}(s), \ldots, \bar{A}_{N}(s)\right) \operatorname{kurz} x=\left(x_{1}, \ldots, x_{N}\right)$ mit $x_{i}:=\bar{A}_{i}(s), i=1, \ldots, N$, und für eine Verteilung $\left(\overline{\mathrm{A}}_{1}(\mathrm{~s}), \ldots, \overline{\mathrm{A}}_{N}(\hat{\mathrm{S}})\right) \operatorname{kurz} \mathrm{y}=\left(\mathrm{y}_{1}, \ldots, \mathrm{y}_{N}\right) \operatorname{mit} y_{i}:=\overline{\mathrm{A}}_{i}(\hat{\mathrm{s}})$, $i=1, \ldots, N$.

Die Lorenzkurve einer beliebigen geordneten Verteilung $\mathbf{x}=\left(x_{1}, \ldots . x_{N}\right)$ der normierten Finanzanteile besteht aus dem Polygonzug, der die Punkte

$$
\left(k, \sum_{i=1}^{k} x_{i}\right) \cup(0,0), \quad k=1, \ldots, N
$$

verbindet.

Ein Punkt

$$
\left(k, \sum_{i=1}^{k} x_{i}\right), \quad k \in\{1, \ldots, N\}
$$

besagt, daß die $k$ Gemeinden mit den geringsten Finanzantei- 
len zusammen einen Anteil an der Summe der normierten Finanzausstattungen von

$$
\sum_{i=1}^{k} x_{i}
$$

haben. Die Lorenzkurve einer Verteilung $x$ geht also durch den Punkt $(N, 1)$, weil alle Gemeinden zusammen

$$
\sum_{i=1}^{N} x_{i}=1
$$

erhalten. Der Punkt $(0,0)$ wird nur aus technischen Gründen benötigt. Man könnte ihn etwa so interpretieren, daß 0 Gemeinden zusammen einen normierten Anteil von 0 erhalten.

Wenn jede Gemeinde $i=1, \ldots, N$ den durchschnittlichen normierten Finanzanteil, also

$$
\frac{1}{N} \sum_{i=1}^{N} x_{i}=\frac{1}{N}
$$

erhält, dann ist die Lorenzkurve die Gerade durch die Punkte $(0,0)$ und $(N, 1)$. Sie nennt man auch die Linie der Gleichverteilung, weil irgend $k$ Gemeinden zusammen genau einen Anteil von

$$
\sum_{i=1}^{k} x_{i}=\frac{k}{N}
$$

erhalten.

Falls für mindestens ein $i$

$$
x_{i} \neq \frac{1}{N}
$$


gilt, dann ist die Lorenzkurve, wie man leicht zeigt, konvex und verläuft "unterhalb" der Linie der Gleichverteilung. Es ist nun naheliegend, als Maß für die Ungleichheit einer Verteilung $x$ grob gesprochen die Abweichung ihrer Lorenzkurve von der Linie der Gleichverteilung anzusehen. Dabei wird eine Verteilung als umso ungleicher eingeschätzt, je weiter sie von der Linie der Gleichverteilung entfernt ist. Wir wollen diese Vorstellung von Ungleichheit nun präzisieren und folgende binäre Relation L definieren:

\section{(3.3.5) Lorenzpräferenz}

Seien $x, y \in \mathbb{R}_{++}^{N}$ irgendzwei geordnete Verteilungen der normierten Finanzanteile und L eine binäre Relation auf $\mathbb{R}_{++}^{N} \times \mathbb{R}_{++}^{N}$.

Genau dann gilt xLy mit der Interpretation "die Verteilung $x$ ist mindestens so gleich wie die Verteilung $y^{\prime \prime}$, wenn

$\sum_{i=1}^{k} x_{i} \geqq \sum_{i=1}^{k} y_{i} \quad$ für alle $k=1, \ldots, N-1$

gilt, d.h. wenn die Lorenzkurve von $x$ überall nicht unterhalb von $y$ liegt.

Die Lorenzpräferenz besagt also, der Anteil der k Ärmsten bei der Verteilung $\mathbf{x}$ ist mindestens so groß wie der Anteil der $k$ Ärmsten bei der Verteilung $y, k=1, \ldots, N-1$.

Gilt in (3.3.5) statt " $\geqq$ " die Relation " $>$ ", dann liegt die Lorenzkurve von $\mathbf{x}$ überall oberhalb der Lorenzkurve von $y_{\text {, }}$ und man erhält die zur Relation L gehörige Lorenzdominanz $L_{\mathrm{p}}$ mit der Interpretation: 


$$
\begin{aligned}
& \mathrm{xL}_{\mathrm{P}} \mathrm{y}-\text { "die Verteilung von } \mathrm{x} \text { ist (echt) gleicher } \\
& \text { als die Verteilung von } \mathrm{y} \text { ". }
\end{aligned}
$$

Gilt in (3.3.5) statt " $\geqq$ " die Relation "=", dann sind die Lorenzkurven von $x$ und $y$ identisch. Die zugehörige Lorenzindifferenz $L_{I}$ hat die Interpretation

$$
\begin{aligned}
& \mathrm{xL}_{\mathrm{I}} \mathrm{Y} \cdots \text { "die Verteilung } \mathrm{x} \text { ist so gleich wie die } \\
& \text { Verteilung } \mathrm{y} " \text {. }
\end{aligned}
$$

Man beachte, bei der Lorenzpräferenz (und bei $L_{P}, L_{I}$ ) wird nicht verlangt, daß die $k$ ärmsten Gemeinden bei der verteilung $x$ identisch sind mit den $k$ ărmsten Gemeinden bei der verteilung $y$. Es ist nur verlangt, daß die Gemeinden bei der Verteilung $x$ nach ihrem normierten Finanzanteil und bei der Verteilung $y$ nach ihrem normierten Finanzanteil geordnet sind.

Das erkennt man sehr schön, wenn die Gemeinden so numeriert sind, daß $x_{i}$ der Finanzanteil der Gemeinde $i$ in der geordneten Verteilung $x=\left(x_{1}, \ldots, x_{N}\right)$ ist. Sei $y_{\pi(i)}$ der Finanzanteil der Gemeinde $i$ in der geordneten Verteilung $y$. Dann ist $\pi(i)$ der Rang der Gemeinde $i$ in $y$ und die Permutation

$$
\begin{aligned}
\pi:\{1, \ldots, N\} & \rightarrow\{1, \ldots, N\} \\
i & \rightarrow \pi(i)
\end{aligned}
$$

ist für $i, j \in\{1, \ldots, N\}$ durch

$$
\begin{aligned}
& y_{\pi(i)}<Y_{\pi(j)} \Rightarrow \pi(i)<\pi(j) \\
& Y_{\pi(i)}=y_{\pi(j)} \Rightarrow \pi(i)<\pi(j) \vee \pi(i)>\pi(j)
\end{aligned}
$$

bestimmt.

Die Lorenzpräferenz (ebenso die dominanz und indifferenz) 
läßt sich auch aus Annahmen ableiten, die sehr viel leichter nachprüfbar, aber auch wesentlich restriktiver sind. Dies besagt der Satz

(3.3.6) Sei $x$ eine geordnete Vertellung der normierten Finanzanteile. Sei $x_{i}$ der normierte Finanzanteil von $i$ in $x$. Sei $y_{\pi(i)}$ der normierte Finanzanteil der Gemeinde 1 in der geordneten Verteilung $y$.

Ist die Permutation

$$
\begin{aligned}
& \pi:\{1, \ldots, N\} \rightarrow\{1, \ldots, N\} \\
& i \rightarrow \pi(i)
\end{aligned}
$$

durch

$$
\pi(i)=i \text { für alle } 1=1, \ldots, N
$$

bestimmt, und existiert ein $1_{0} \in\{1, \ldots, N-1\}$ mit

$$
\begin{aligned}
& x_{i} \geq y_{i}, i=1, \ldots, 1_{0} \\
& x_{i} \leqq y_{1}, i=1_{0}+1, \ldots, N,
\end{aligned}
$$

dann ist $\mathrm{xLy}$.

Ersetzt man in (3.3.6) die Relationen " $x_{i} \geq y_{i}$ " und " $x_{i} \leqq y_{i}$ "durch $" x_{i}=y_{i} "$, dann folgt die Lorenzindifferenz. Für die Lorenzdominanz muß zusätzlich zu den Voraussetzungen in (3.3.6) noch

$$
\text { (3.3.7) } \quad x_{1}>y_{1} \wedge x_{N}<y_{N}
$$

gelten.

Das Kriterium (3.3.6) läßt sich sehr anschaulich etwa so 
interpretieren: Ist der Rang jeder Gemeinde in der geordneten Verteilung $y$ der gleiche wie in der geordneten Verteilung $x$, gilt also

$$
x_{1} \leq x_{2} \leq \cdots \leq x_{N} \Rightarrow y_{1} \leq y_{2} \leq \cdots \leq y_{N}
$$

und hat jede der $i_{0}$ ärmsten Gemeinden bei $x$ einen nicht niedrigeren normierten Finanzanteil als bei $y$ und jede der $\left(\mathrm{N}-\mathrm{i}_{0}\right)$ reichsten Gemeinden bei $x$ einen nicht höheren Finanzanteil als bei $y$, dann ist $x$ mindestens so gleich wie $y$.

Die leichtere Praktikabilität von Kriterium (3.3.6) wird damit erkauft, daß man es nur auf Verteilungen anwenden kann, in denen sich der Rang der Gemeinden nicht ändert. Es wird sich aber zeigen, daß diese Voraussetzung bei den Verteilungen, mit denen wir es zu tun haben werden, immer erfüllt ist. So können wir uns die Arbeit erheblich erleichtern, wenn wir statt (3.3.5) das Kriterium (3.3.6) anwenden, ja man könnte sogar sagen, daß uns Aussagen über die Progressivität oder Regressivität von Verteilungen durch die Umformulierung von (3.3.5) erst ermöglicht werden, denn wir wollen solche Aussagen nicht empirisch, sondern deduktiv gewinnen.

Natürlich werden wir (3.3.6) auch anwenden, wenn wir die Verteilung der normierten steuerkraft mit einer beliebigen Verteilung der normierten Finanzausstattungen vergleichen. Die Vektoren $x$ und $y$ sind dann entsprechend $z u$ spezifizieren (siehe 4.2).

Beweis von $(3.3 .6)$ und $(3.3 .7)$

Sei $x_{i}, y_{i}$ der Anteil von $i$ bei $x$ bzw. $y$.

Sei $x_{1} \leq x_{2} \leq \ldots \leq x_{N} \Rightarrow y_{1} \leq \ldots \leq y_{N}$ 
Es existiere ein $i_{0}$ mit

$$
x_{i} \geq y_{i} \quad \text { für } i=1, \ldots, i_{0} .
$$

Dann ist

$$
\sum_{i=1}^{k} x_{i} \geqq \sum_{i=1}^{k} y_{i}, k=1, \ldots, i_{0}
$$

Es gelte

$$
x_{i} \leqq y_{1} \quad \text { für } i=i_{0}+1, \ldots, N
$$

Dann ist wegen $\sum_{i=1}^{N} x_{i}=\sum_{i=1}^{N} Y_{i}$

$$
\sum_{i=1}^{N-k} x_{i} \geqq \sum_{i=1}^{N-k} y_{i} \quad, k=1, \ldots, i_{0}-1 .
$$

Zusammengenommen gilt also

$$
\sum_{i=1}^{k} x_{i} \geqq \sum_{i=1}^{k} y_{i}, k=1, \ldots, N-1,
$$

und daraus folgt xLy.

Ist zusätzlich $x_{1}>y_{1}$ und $x_{N}<y_{N^{\prime}}$

dann ist

$$
\sum_{i=1}^{k} x_{i}>\sum_{i=1}^{k} y_{i}, k=1, \ldots, i_{0}
$$

und 


$$
\sum_{i=1}^{N-k} x_{i}>\sum_{i=1}^{N-k} Y_{i}, \quad k=1, \ldots, i_{0}-1,
$$

insgesamt also

$$
\sum_{i=1}^{k} x_{i}>\sum_{i=1}^{k} y_{i}, k=1, \ldots, N-1,
$$

und somit $x L_{p} Y$.

口

In der nun folgenden Analyse werden Verteilungen der Schlüsselzuweisungen aus staatlicher Sicht insbesondere danach beurteilt, ob fiskalische Ungleichheit verringert oder ganz beseitigt werden kann. Aus kommunaler Sicht interessiert vor allem die Frage, ob eine Verteilung für eine Gemeinde, gemessen an ihrer optimalen Verteilung, eher günstig oder ungünstig ist. Dazu vergleichen wir die Verteilungswirkungen der Schlüsselzuweisungen mit einer situation, in der die Kommunen nur über ihre eigene steuerkraft verfügen können. Danach untersuchen wir alternative Verteilungen, die durch eine Variation der Schlüsselmasse und der Ausschüttungsquote entstehen. In die Analyse werden schlieblich auch Sonderschlüsselzuweisungen und Mindestgarantien einbezogen. 
I. I VERTEILUNGSANALYSE

\section{Die fiskalische Ungleichheit der Gemeinden}

In diesem Kapitel vergleichen wir die Verteilungsergebnisse der Schlüsselzuweisungen mit einer situation, in der die Gemeinden nur über ihre eigene Steuerkraft verfügen können. Dazu gehen wir von einer beliebigen Verteilung $\mathbf{s}=\left(s_{1}, \ldots s_{N}\right)$ mit den schlüsselzuweisungen

$(4.1)$

$$
s_{i=1, \ldots, N} \begin{cases}0 & , G(s) \leqq \bar{K}_{i} \\ a\left(B_{i}(s)-k_{i}\right), G(s)>\bar{k}_{i}\end{cases}
$$

aus, wobei Gis) den (eindeutig bestimmten) Grundbetrag bei der Verteilung s bezeichnet. Dann existiert bei s bekanntlich eine Gemeinde $n(s) \in\{1, \ldots, N\}$ mit

$$
\begin{aligned}
& G(s)>\overline{\mathrm{K}}_{i}, i=1, \ldots, \mathrm{n}(\mathrm{s}) \\
& G(s) \leqq \overline{\mathrm{K}}_{i}, i=n(s)+1, \ldots, \mathrm{N},
\end{aligned}
$$

wenn wir zusätzlich noch annehmen, daß die Gemeinden nach ihrer normierten steuerkraft geordnet sind.

Der Einfachheit halber schreiben wir in den Abschnitten 4.1 und 4.2 für $\mathrm{n}(\mathrm{s}), \mathrm{G}(\mathrm{s}), \mathrm{B}_{i}(\mathrm{~s})$ und auch für $\mathrm{F}_{i}(\mathrm{~s}), \mathrm{D}_{i}(\mathrm{~s})$, $A_{i}$ (s) $\operatorname{kurz} n, G, B_{i}, F_{i}, D_{i}, A_{i}$, weil nur eine Verteilung betrachtet wird und Verwechslungen nicht zu befürchten sind. Dann vereinfacht sich (4.1) unter Beachtung von (4.2) $\mathrm{zu}$

$$
s_{i=1, \ldots, N} \begin{cases}0, & i=n+1, \ldots, N \\ a\left(B_{i}-k_{i}\right), & i=1, \ldots, n .\end{cases}
$$




\subsection{Finanzierungsdefizite und Finanzierungsüberschüsse in den Gemeinden}

Addiert man zur Steuerkraft einer Gemeinde $i$ ihre Schlüsselzuweisung aus (4.3), so erhält man die Finanzausstattung

(4.1.1) $\quad F_{i}= \begin{cases}k_{i}, & i=n+1, \ldots, N \\ a B_{i}+(1-a) k_{i}, & i=1, \ldots, n .\end{cases}$

In eher reichen Gemeinden entspricht die Finanzausstattung also der Steuerkraft. Eher arme Gemeinden verfügen über eine Linearkombination aus steuerkraft und Bedarf, wobei in diesen Gemeinden für eine Verteilung mit $0<a<1$ und unter der Voraussetzung $B_{i} \neq K_{i}$ (die aber immer erfüllt ist) gilt:

(4.1.2) $\min \left\{B_{i}, K_{i}\right\}<F_{i}<\max \left\{B_{i}, K_{i}\right\}$

und folglich

(4.1.3) $\quad \mathrm{K}_{i}<\mathrm{F}_{i}<\mathrm{B}_{i}, \quad i=1, \ldots, \mathrm{n}$.

Obwohl in eher armen Gemeinden die finanziellen Mittel höher als die eigenen steuereinnahmen sind, reichen sie nicht aus, den Finanzbedarf zu decken. In eher reichen Gemeinden sind sie dagegen auch ohne zuweisungen nicht geringer als der Finanzbedarf, weil

$$
\text { (4.1.4) } \quad F_{i} \geqq B_{i}, i=n+1, \ldots, N
$$

gilt.

Bei Verteilungen mit einem Ausgleichssatz von $a=1$ ist die Finanzausstattung 
(4.1.5) $\quad F_{i}=\left\{\begin{array}{l}k_{i}, i=n+1, \ldots, N \\ B_{i}, i=1, \ldots, n\end{array}\right.$

und daher

$(4.1 .6) \quad F_{i}=\max \left\{B_{i}, K_{i}\right\}, i=1, \ldots, N$.

Die Finanzausstattung ist in allen Gemeinden also mindestens so hoch wie der Bedarf, in reichen Gemeinden ist sie meist sogar höher. Ein Ausgleichssatz von Eins wird vielfach abgelehnt, ${ }^{1)}$ weil er den Gemeinden keinen Anreiz biete, ihre eigenen steuerquellen auszuschöpfen. Wir werden aber sehen, daß man unter redistributiven Gesichtspunkten $z u$ einer ganz anderen Einschätzung kommt (s. 4.3 und 6).

Das Finanzierungsdefizit einer Gemeinde i beträgt

(4.1.7) $\quad D_{i}= \begin{cases}B_{i}-K_{i}, & i=n+1, \ldots, N \\ (1-a)\left(B_{i}-K_{i}\right), & i=1, \ldots, n .\end{cases}$

In eher reichen Gemeinden ist $D_{i} \leqq 0, d . h$. diese Gemeinden haben zumindest kein Finanzierungsdefizit, meist sogar einen Finanzierungsüberschuß. Eher arme Gemeinden haben bei einer Verteilung mit $a<1$ ein Finanzierungsdefizit, das aber geringer ist als das Defizit zwischen Finanzbedarf und Steuerkraft. Für Verteilungen mit $a=1$ ist in diesen Gemeinden $D_{i}=0$, sie werden aber nie einen Finanzierungsüberschuß aufweisen.

Um die Finanzausstattung und das Finanzierungsdefizit der

1) Siehe Bchley, Foohs [1984], IV 10, Anliang zu Art. 1-6, S. 1; Neumark [1978], S. $167 \mathrm{ff.;}$ Smekal [1980], S. 194; Weber [1981], S. $74 \mathrm{ff}$. 
Gemeinden sinnvoll vergleichen zu können, benötigt man die normierten Größen. Wir erhalten für eine Verteilung mit $0<a<1$ die normierte Finanzausstattung der Gemeinden als

(4.1.8) $\quad \bar{F}_{i}= \begin{cases}\bar{k}_{i^{\prime}} & i=n+1, \ldots, N \\ a G+(1-a) \bar{k}_{i^{\prime}} & i=1, \ldots, n .\end{cases}$

Bemerkenswert hier ist, daB die armen Gemeinden eine Linearkombination aus ihrer normierten steuerkraft und einem für alle Gemeinden einheitlichen Betrag G bekommen.

Folglich ist

(4.1.9) $\quad \bar{F}_{i}\left\{\begin{array}{l}\geq G, \quad i=n+1, \ldots, N \\ <G, \quad i=1, \ldots, n .\end{array}\right.$

Nimmt man G als Vergleichsgröße, so ist in reichen Gemeinden die normierte Finanzausstattung nicht kleiner als G, während sie in armen Gemeinden immer darunter liegt.

Entsprechend folgt für das normierte Finanzierungsdefizit

$(4.1 .10) \quad \bar{D}_{i}= \begin{cases}G-\bar{K}_{i}, & i=n+1, \ldots, N \\ (1-a)\left(G-\bar{K}_{i}\right), & i=1, \ldots, n\end{cases}$

und somit

$$
\bar{D}_{i} \quad \begin{cases}\leqq 0, & i=n+1, \ldots, N \\ >0, & i=1, \ldots, n\end{cases}
$$

Für eine Verteilung mit $a=1$ ist 
(4.1.11) $\quad \bar{F}_{i}= \begin{cases}\bar{K}_{i}, & i=n+1, \ldots, N \\ G, & i=1, \ldots, n\end{cases}$

und

$(4.1 .12) \quad \bar{D}_{i} \quad\left\{\begin{array}{l}\leqq 0, \quad i=n+1, \ldots, N \\ =0, \quad i=1, \ldots, n .\end{array}\right.$

Man sieht, die normierte Finanzausstattung ist in armen Gemeinden gleichverteilt und entspricht dem normierten Finanzbedarf, folglich ist in diesen Gemeinden das normierte Finanzierungsdefizit Null. Es besteht aber keine fiskalische Gleichheit, da steuerstarke Gemeinden nach wie vor FiFinanzierungsüberschüsse aufweisen können.

Abschließend vergleichen wir den Finanzanteil A, einer Gemeinde mit ihrem Steuerkraftanteil. Allgemein gilt für $i=1, \ldots, \mathrm{N}$ :

$(4.1 .13) \quad A_{i}>\frac{K_{i}}{K} \Leftrightarrow \frac{S_{i}}{M}>\frac{K_{i}}{K}$.

Der Finanzanteil einer Gemeinde $i$ ist also genau dann höher als ihr Steuerkraftanteil, wenn der Antell ihrer Schlüsselzuweisungen an der Schlüsselmasse, d.h. ihr Zuweisungsanteil höher als der steuerkraftanteil ist.

Für $i=n+1, \ldots, N$ gilt:

(4.1.14) $\quad \frac{S_{i}}{M}=0 \Rightarrow A_{i}<\frac{K_{i}}{K}$.

Damit gibt es in der Gruppe $\{1, \ldots, n\}$ mindestens ein $i$ mit

$$
A_{i}>\frac{K_{i}}{K} \text {. }
$$

Man erhält für $\underline{i}=1, \ldots, n:$ 


$$
A_{i}>\frac{K_{i}}{K} \Leftrightarrow \frac{S_{i}}{M}>\frac{K_{i}}{K} \Leftrightarrow \bar{K}_{i}<\frac{G \cdot K}{\frac{M}{a}+K} .
$$

Dabei ist

$$
\frac{G \cdot K}{\frac{M}{a}+K}<G \leftrightarrow \frac{M}{a}>0 \text {, }
$$

was nach Voraussetzung immer erfüllt ist. Es gibt also eine Gemeinde $i_{0} \in\{1, \ldots, n\}$ mit

$$
\begin{aligned}
& A_{i}>\frac{K_{i}}{K} \quad, \quad i=1, \ldots, i_{0} \\
& A_{i} \leqq \frac{K_{i}}{K} \quad, \quad i=i_{0}+1, \ldots, n .
\end{aligned}
$$

Bemerkenswert hier ist, daß nicht unbedingt alle Gemelnden, die zuweisungsberechtigt sind, einen Finanzanteil aufweisen, der höher als ihr steuerkraftanteil ist.

\subsection{Die Nichtregressivität der Schlüsselzuweisungen}

Wir untersuchen nun, ob eine Verteilung nichtregressiv ist, also Kriterium (3.1.12) erfüllt. Dazu müssen wir die zu einer Verteilung $\mathbf{s}$ der Schlüsselzuweisungen gehörige Verteilung der normierten Finanzausstattungen mit der Verteilung der Steuerkraft vergleichen. Da

$$
\sum_{i=1}^{N} \overline{\mathrm{K}}_{i}<\sum_{i=1}^{N} \overline{\mathrm{F}}_{i}
$$

ist, rekurrieren wir auf die normierten Finanzanteile bzw. Steuerkraftanteile.

Sei also in der geordneten Verteilung $x=\left(x_{1}, \ldots, x_{N}\right)$

$$
\text { (4.2.1) } \quad x_{i}:=\frac{\overline{\mathrm{K}}_{1}}{\sum_{j=1}^{\mathrm{N}} \overline{\mathrm{K}}_{j}}, \quad i=1, \ldots, \mathrm{N}
$$


der normierte Steuerkraftanteil der Gemeinde i, und sei (4.2.2) $y_{i}:=\frac{\bar{F}_{i}}{\sum_{j=1}^{N} \bar{F}_{j}}, \quad i=1, \ldots, N$

der normierte Finanzanteil der Gemeinde 1 in der (ungeordneten) Verteilung $y=\left(y_{1}, \ldots, y_{N}\right)$.

Zunächst ist zu zeigen (s. $(3.3 .6))$ :

(4.2.3) $\quad x_{1} \leqq x_{2} \leqq \cdots \leqq x_{N} \Rightarrow y_{1} \leqq y_{2} \leqq \cdots \leqq y_{N}$.

Dies gilt genau dann, wenn

(4.2.4) $\quad \overline{\mathrm{K}}_{1} \leq \overline{\mathrm{K}}_{2} \leq \ldots \leq \overline{\mathrm{K}}_{\mathrm{N}} \Rightarrow \overline{\mathrm{F}}_{1} \leq \overline{\mathrm{F}}_{2} \leq \ldots \leq \overline{\mathrm{F}}_{\mathrm{N}}$

erfüllt ist.

Beweis:

Für $i=2, \ldots, n$ gilt:

$$
\begin{aligned}
& \overline{\mathrm{F}}_{i-1} \leqq \overline{\mathrm{F}}_{i} \\
& \text { - } \mathrm{aG}+(1-\mathrm{a}) \overline{\mathrm{K}}_{i-1} \leq \mathrm{aG}+(1-\mathrm{a}) \overline{\mathrm{K}}_{i} \\
& \text { - } \overline{\mathrm{K}}_{i-1} \leqq \overline{\mathrm{K}}_{i} .
\end{aligned}
$$

Falls bei einer Verteilung $s \in A=N$ gilt, ist $(4.2 .4)$ gezeigt. Für Verteilungen mit $\mathrm{n}<\mathrm{N}$ gilt zusätzlich:

Für $i=n+1, \ldots, N-1$ :

$$
\bar{F}_{i}=\bar{K}_{i} \leqq \bar{K}_{i+1}=\bar{F}_{i+1} \Rightarrow \bar{F}_{i} \leqq \bar{F}_{i+1} .
$$

Bleibt zu zeigen: 


$$
\bar{F}_{n} \leqq \bar{F}_{n+1}
$$

Aus $\overline{\mathrm{F}}_{\mathrm{n}}=\mathrm{aG}+(1-\mathrm{a}) \overline{\mathrm{K}}_{\mathrm{n}} \leqq \max \left\{\mathrm{G}, \overline{\mathrm{K}}_{\mathrm{n}}\right\}=\mathrm{G} \quad$ für $0 \leqq a \leqq 1$

folgt

$$
\bar{F}_{n} \leq G \leq \bar{K}_{n+1}=\bar{F}_{n+1} \Rightarrow \bar{F}_{n} \leqq \bar{F}_{n+1} .
$$

Halten wir fest: Bei einer beliebigen Verteilung $s \in A$ ist die Rangordnung der Gemeinden nach ihrer normierten Finanzausstattung immer identisch zur Ordnung, die aus der normierten Steuerkraft resultiert. Damit ist die normierte Finanzausstattung einer Gemeinde $i$ also nie größer als die normierte Finanzausstattung einer Gemeinde $j$, wenn die Gemeinde $i$ nicht reicher als $j$ ist, $d . h$. eine höhere steuerkraft aufweist. Die Ordnung der Gemeinden nach ihrer Armut kann also mit Schlüsselzuweisungen nicht verändert werden, ganz gleich, welche Verteilung gewählt wird.

Wir vergleichen nun den normierten Finanzanteil einer jeden Gemeinde mit ihrem normierten steuerkraftanteil.

Ganz allgemein gilt für $i=1, \ldots, N$ :

$$
x_{i}<y_{i} \leftrightarrow x_{i}<s_{i}
$$

wobei

$$
s_{i}:=\frac{\bar{s}_{i}}{\sum_{j=1}^{\mathbb{N}} \bar{s}_{j}}
$$

der Anteil der normierten Schlüsselzuweisung der Gemeinde i an der summe aller normierten Schlüsselzuweisungen, kurz der normierte Zuweisungsanteil ist. Ist er höher als der normierte steuerkraftanteil, dann ist auch der normierte Finanzanteil der Gemeinde $i$ höher als der steuerkraftanteil. 
Für $i=n+1, \ldots, N$ :

(4.2.5) $s_{i}=0 \Rightarrow x_{i}>s_{i} \Rightarrow x_{i}>y_{i}$.

Wegen $\sum_{i=1}^{N} x_{i}=\sum_{i=1}^{N} y_{i}=1$ existiert daher mindestens ein $i \in\{1, \ldots, n\}$ mit $x_{1}<y_{1}$.

Für $i=1, \ldots, n:$

$$
\begin{gathered}
x_{i}<s_{i}-\frac{\overline{\mathrm{K}}_{i}}{\sum_{j=1}^{N} \overline{\mathrm{K}}_{j}}<\frac{a\left(G-\overline{\mathrm{K}}_{i}\right)}{\sum_{j=1}^{N} \bar{s}_{j}} \\
\leftrightarrow \quad \overline{\mathrm{k}}_{i}<\frac{G \sum_{j=1}^{N} \overline{\mathrm{K}}_{j}}{n G+\sum_{j=n+1}^{N} \overline{\mathrm{K}}_{j}} .
\end{gathered}
$$

Dabei ist

$$
\frac{G \sum_{j=1}^{N} \overline{\mathrm{K}}_{j}}{n G+\sum_{j=n+1}^{N} \overline{\mathrm{K}}_{j}} \leqq G-\frac{1}{\mathrm{n}} \sum_{j=1}^{n} \overline{\mathrm{K}}_{j} \leqq G,
$$

was aber immer erfüllt ist, da

$$
\mathrm{G}>\overline{\mathrm{K}}_{\mathrm{i}}, \mathrm{i}=1, \ldots, \mathrm{n}
$$

und

$$
\frac{1}{n} \sum_{i=1}^{n} \bar{k}_{i} \leqq \max \left(\bar{K}_{1}, \ldots, \bar{k}_{n}\right)
$$

gilt. 
Es existiert also ein $i_{0} \epsilon\{1, \ldots, n\}$ mit

$$
\begin{aligned}
& y_{i}>x_{i}, \quad i=1, \ldots, i_{0} \\
& y_{i} \leqq x_{i}, \quad i=i_{0}+1, \ldots, n .
\end{aligned}
$$

Aus (4.2.3) und $(4.2 .6)$ folgt für Verteilungen $s \in A$ mit $\mathrm{n}(\mathrm{s})=\mathrm{N}$ (also alle Gemeinden sind zuwelsungsberechtigt) nach Kriterium (3.3.6) yLx und somit die Nichtregressivität von s. Verteilungen $\mathbf{s}$ mit $\mathrm{n}(\mathrm{s})<\mathrm{N}$ (wovon man in den meisten praktischen Fällen wohl ausgehen muB) sind sogar progressiver als die Verteilung der normierten steuerkraft, weil zusätzlich mit (4.2.5) yL $\mathrm{L}_{\mathrm{p}} \mathrm{x}$ folgt.

Zusammenfassend läßt sich sagen, mit Schlüsselzuweisungen wird die fiskalische Ungleichheit der Gemeinden zumindest nicht erhöht, meist wird sie sogar geringer. Ob sie allerdings ganz beseitigt werden kann, untersuchen wir im nächsten Abschnitt:

\subsection{Bedingungen für fiskalische Gleichheit}

Gesucht ist eine Verteilung $s^{*} E A$, die fiskalische Gleichheit erzeugt. Eine solche Verteilung wird aber kaum zu finden sein, wenn die Ausschüttungsquote kleiner als Eins sein soll. Denn es läßt sich in diesem Falle zeigen, daß die normierten Finanzausstattungen genau dann gleichverteilt sind, wenn die normierte steuerkraft gleichverteilt ist.

Es gilt also für $s \in \mathbb{A}$ mit $a<1$ :

$(4.3 .1)$

$$
\begin{aligned}
& \overline{\mathrm{K}}_{1}=\overline{\mathrm{K}}_{2}=\ldots=\overline{\mathrm{K}}_{\mathrm{N}} \\
& \overline{\mathrm{F}}_{1}(\mathrm{~s})=\overline{\mathrm{F}}_{2}(\mathrm{~s})=\ldots=\overline{\mathrm{F}}_{\mathrm{N}}(\mathrm{s}) .
\end{aligned}
$$




\section{Beweis:}

1) Sei $\overline{\mathrm{K}}_{1}=\overline{\mathrm{K}}_{2}=\ldots=\overline{\mathrm{K}}_{\mathrm{N}}$. Für $\mathrm{M}>0$ ist $\mathrm{G}(\mathrm{s})>\overline{\mathrm{K}}_{i}$ für mindestens ein $i \in\{1, \ldots, N\}$. Wegen der Gleichverteilung der $\overline{\mathrm{K}}_{i}$ ist

$G(s)>\overline{\mathrm{K}}_{i}$ für $i=1, \ldots, \mathrm{N}$

$\rightarrow \bar{F}_{i}(s)=a G(s)+(1-a) \bar{K}_{i,} i=1, \ldots, N$

$\rightarrow \bar{F}_{i}(s)=\bar{F}_{j}(s), i, j \in\{1, \ldots, N\}$.

2) Sei $\bar{F}_{1}(s)=\bar{F}_{2}(s)=\ldots=\bar{F}_{N}(s)$.

Es sind drei Fälle zu unterscheiden:

a) $s$ ist eine Verteilung mit $G(s) \leq \bar{K}_{i}, i=1, \ldots, N$. Dieser Fall kann nicht eintreten, da $G(s)>\bar{K}_{i}$ ist für mindestens ein $i \in\{1, \ldots, N\}$ und alle $s \in \mathbb{A}$.

b) $s$ ist eine Verteilung mit $G(s)>\bar{k}_{i}, i=1, \ldots, n(s)$ und $n:=n(s)<N$. Dieser Fall führt zum Widerspruch zur Voraussetzung, denn es gilt:

$$
\begin{aligned}
& \bar{F}_{n}(s)=a G(s)+(1-a) \bar{K}_{n}<\max \left\{G(s), \bar{K}_{n}\right\}=G(s), \\
& \bar{F}_{n+1}(s)=\bar{K}_{n+1} \geq G(s) \\
& \rightarrow \bar{F}_{n}(s)<\bar{F}_{n+1}(s) .
\end{aligned}
$$

c) $\mathbf{s}$ ist eine Verteilung $\mathrm{mit} G(\mathrm{~s})>\overline{\mathrm{K}}_{i}, i=1, \ldots, \mathrm{N}$.

$$
\begin{aligned}
& \bar{F}_{i}(s)=\bar{F}_{j}(s) \\
& a G(s)+(1-a) \bar{K}_{i}=a G(s)+(1-a) \bar{K}_{j} \\
& \bar{K}_{i}=\bar{K}_{j}, i ; j \in\{1, \ldots, N\} .
\end{aligned}
$$


Fiskalische Gleichheit impliziert also die Gleichverteilung der normierten Steuerkraft. Dieses Resultat ist unter Verteilungsgesichtspunkten ziemlich ernüchternd, denn es besagt auch, wenn die normierte steuerkraft der Gemeinden nicht gleichverteilt ist (was praktisch immer der Fall ist), dann gibt es auch keine fiskalische Gleichheit. Sollte die normierte steuerkraft (rein theoretisch) dennoch einmal gleichverteilt sein, dann sind Schlüsselzuweisungen für redistributive Zwecke nicht nötig (fiskalische Gleichheit ist ja schon gegeben), sie dienen allenfalls zur Aufstockung der gemeindlichen steuerkraft (fiskalische Funktion). Wenigstens erhalten die Schlüsselzuweisungen dabei die fiskalische Gleichheit der Gemeinden, was wohl als Minimalforderung gelten kann.

Bei einem Ausgleichssatz von $a=1$ gibt es Verteilungen, mit denen fiskalische Gleichheit auch dann erzeugt werden kann, wenn die normierte steuerkraft ungleich verteilt ist.

Sei also

$$
\begin{aligned}
& \overline{\mathrm{K}}_{1} \leqq \overline{\mathrm{K}}_{2} \leqq \cdots \leqq \overline{\mathrm{K}}_{\mathrm{N}} \text { und } \\
& \overline{\mathrm{K}}_{\mathrm{i}}<\overline{\mathrm{K}}_{\mathrm{i}+1} \text { für mindestens ein } i \in\{1, \ldots, \mathrm{N}-1\} .
\end{aligned}
$$

Für $s \in A$ und $a=1$ ergibt $\operatorname{sich}(s .(4.1 .11)):$

$$
\bar{F}_{i}(s)=\left\{\begin{array}{l}
G(s), i=1, \ldots, n(s) \\
\bar{K}_{i}, i=n(s)+1, \ldots, N .
\end{array}\right.
$$

Die normierte Finanzausstattung ist also, unabhängig von der Steuerkraft, in allen zuweisungsberechtigten Gemeinden gleich. Eine Verteilung $s^{*} \in A$ erzeugt also fiskalische Gleichheit, falls 
(4.3.2) $G\left(s^{*}\right)>\bar{k}_{i}$ für alle $i=1, \ldots, N$

gilt, d.h. $n\left(s^{*}\right)=\mathrm{N}$. Dann ist

$$
\bar{F}_{i}\left(s^{*}\right)=G\left(s^{*}\right) \Rightarrow \bar{D}_{i}\left(s^{*}\right)=0 \text { für alle } i=1, \ldots, N \text {. }
$$

Damit bei einem Ausgleichssatz von $a=1$ alle Gemeinden zuweisungsberechtigt sind, muß die Schlüsselmasse einen bestimmten Betrag überschreiten, den wir in 6.1 noch spezifizieren werden. Dieser Betrag dürfte allerdings recht hoch sein, denn in der Praxis reicht die verfügbare Schlüsselmasse sogar bei einem Ausgleichssatz von a $<1$ nicht aus, um allen Gemeinden Zuweisungen zu gewähren. 


\section{Verteilungen bei variabler Schlüsselmasse}

Bisher sind wir davon ausgegangen, daß die exogenen Größen, so auch die Schlüsselmasse, gegeben sind. Daraus resultiert bekanntlich eine eindeutige Verteilung der Schlüsselzuweisungen. Wenn man aber die Schlüsselmasse als Parameter ansieht, so kann man bei ihrer Variation alternative Verteilungen $\mathbf{s}(M)=\left(S_{1}(M), \ldots, S_{N}(M)\right)$ erzeugen, bei denen die Schlüsselzuweisungen der Gemeinden von der Schlüsselmasse abhängen. Dies setzt natürlich einen funktionalen zusammenhang zwischen den Schlüsselzuweisungen und der Schlüsselmasse voraus, letztlich also einen funktionalen zusammenhang zwischen dem Grundbetrag und der Schlüsselmasse, denn alle anderen Größen sind exogen und von der Schlüsselmasse unabhängig.

\subsection{Die Abhängigkeit der Schlüsselzuwelsungen von der} Schlüsselmasse

Die Schlüsselzuweisung einer Gemeinde $i$

$(5.1 .1)$

$$
\begin{array}{ll}
S_{i}(G(M))=\Theta_{i}(G(M)) a\left(E_{i} b_{i} G(M)-K_{i}\right) \\
\text { mit } & \Theta_{i}(G(M))=\left\{\begin{array}{cc}
0, & \bar{K}_{i} \geqq G(M) \\
1, & \bar{K}_{i}<G(M)
\end{array}\right.
\end{array}
$$

ist hier (über $G(M)$ ) nur indirekt von der Schlüsselmasse abhängig. Für die Darstellung der direkten Abhängigkeit sind wir zunächst an einer Funktion

$$
(5.1 .2) \quad G: M \rightarrow G(M)
$$


interessiert, die jeder Schlüsselmasse einen eindeutigen Grundbetrag als Funktionswert von G zuordnet. Die Funktionen

(5.1.3) $s_{i}: M \rightarrow s_{i}(M), i=1, \ldots, N$

erhalten wir dann durch Einsetzen von (5.1.2) in (5.1.1) und Elimination der Dummy-Variablen.

\subsubsection{Der Grundbetrag als Funktion der Schlüsselmasse}

Ist eine bestimmte Schlüsselmasse $M_{0}$ gegeben, so existiert bekanntlich ein eindeutiger Grundbetrag

$$
\text { (5.1.4) } G_{0}:=G\left(M_{0}\right)=\frac{\frac{M_{0}}{a}+\sum_{i=1}^{n_{0}} K_{i}}{\sum_{i=1}^{n_{0}} E_{i} b_{i}} .
$$

Dabei ist vorausgesetzt, daß die Gemeinden nach ihrer normierten Steuerkraft in aufsteigender Reihenfolge geordnet sind und $n_{0}:=n\left(G\left(M_{0}\right)\right)$ diejenige Gemeinde ist, die bei $M_{0}$ gerade noch Zuweisungen erhält, d.h. es gilt

$$
\begin{aligned}
& (5.1 .5) \quad E_{n_{0}} b_{n_{0}} G_{0}>K_{n_{0}} \\
& (5.1 .6) \quad E_{n_{0}+1} b_{n_{0}+1} G_{0} \leqq K_{n_{0}+1} .
\end{aligned}
$$

Man kann sich nun leicht vorstellen, daß sich mit der Schlüsselmasse auch der Kreis der zuweisungsberechtigten Gemeinden ändern kann, und zwar wird er, wie wir gleich zeigen, meist größer, wenn die Schlüsselmasse steigt und klei- 
ner, wenn die Schlüsselmasse sinkt. In einem eng begrenzten Intervall kann aber $M_{0}$ variiert werden, ohne daß sich der Kreis der zuweisungsberechtigten Gemeinden ändert. Eine Untergrenze $M_{0}$ dieses Intervalls erhält man durch Einsetzen von $(5.1 .4)$ in $(5.1 .5)$ und einige Umformungen

(5.1.6a) $M_{0}>a\left(\bar{K}_{n_{0}} \sum_{i=1}^{n_{0}} E_{i} b_{i}-\sum_{i=1}^{n_{0}} K_{i}\right)=: \underline{M}_{0}$

und eine Obergrenze $\bar{M}_{0}$ durch Einsetzen von $(5.1 .4)$ in $(5.1 .6)$

$(5.1 .7)$

$$
M_{0} \leqq a\left(\bar{K}_{n_{0}+1} \sum_{i=1}^{n_{0}} E_{i} b_{i}-\sum_{i=1}^{n_{0}} K_{i}\right)=: \bar{M}_{0}
$$

Mit (5.1.4), (5.1.6a) und (5.1.7) ist $G(M)$ auf einem kleinen Bereich definiert. Sie gibt den Zusammenhang zwischen den Grundbeträgen, bei denen eine bestimmte Gemeinde gerade noch Zuweisungen erhält, und dem Intervall an, in dem sich die Schlüsselmasse dabei bewegen muB. Betrachtet man sukzessiv Grundbeträge, bei denen $n=1, \ldots, N$ Gemeinden Zuweisungen erhalten und gibt dazu jeweils das zugehörige Intervall für $M$ an, dann läßt sich $G(M)$ abschnittsweise definieren:

(5.1.8) $G(M)=\frac{1}{a \sum_{i=1}^{n} E_{i} b_{i}} M+\frac{\sum_{i=1}^{n} K_{i}}{\sum_{i=1}^{n} E_{i} b_{i}} \quad$ für $\quad M \in I_{n}=\left(M_{n}, \bar{M}_{n}\right]$, 
Dabei ist die Untergrenze $\underline{M}_{n}$ des Intervalls $I_{n}, n=1, \ldots, N$ durch

(5.1.9) $\quad \underline{M}_{n}=a\left(\bar{K}_{n} \sum_{i=1}^{n} E_{i} b_{i}-\sum_{i=1}^{n} K_{i}\right)$,

und die obergrenze $\bar{M}_{n}$ von $I_{n}, n=1, \ldots, N-1$ durch

$(5 \cdot 1 \cdot 10) \quad \bar{M}_{n}=a\left(\bar{K}_{n+1} \sum_{i=1}^{n} E_{i} b_{i}-\sum_{i=1}^{n} K_{i}\right)$

bestimmt. $\bar{M}_{N}$ kann durch $(5.1 .10)$ nicht definiert werden, weil $\bar{K}_{\mathrm{N}+1}$ nicht definiert ist. Wir nehmen deshalb einfach an, $\bar{M}_{N}$ sei eine sehr große endliche reelle $\mathrm{Zahl}$, von der wir nur die Eigenschaft

$$
\underline{M}_{N}<\bar{M}_{N}<\infty
$$

fordern. ${ }^{1)}$

Wir zeigen nun: $G(M)$ ist durch $(5.1 .8),(5.1 .9)$ und $(5.1 .10)$ auf dem ganzen Bereich $\left(0, \overline{\mathrm{M}}_{\mathrm{N}}\right)$ definiert, stetig und streng monoton wachsend.

1) $\bar{M}_{N}$ ist die Obergrenze des Definitionsbereichs von $G(M)$ und nicht $z u$ verwechseln mit einer exogenen Beschränkung der Schlüsselmasse auf eine Höhe $\bar{M}<\bar{M}_{N}$ von der Einnahmenseite her (s. 5.2). 


\section{Definitionsbereich:}

Für $n=1$ ist $\underline{M}_{1}=0, d . h$. bei einer positiven Schlüsselmasse bekommt mindestens eine Gemeinde Zuweisungen, was auch ein Beweis für die Behauptung in Abschnitt 2.1.2 ist:

$$
\begin{aligned}
& \theta_{i}=1 \text { für mindestens ein } i \in\{1, \ldots, N\}, \\
& \text { falls } M>0 \text { ist. }
\end{aligned}
$$

Bleibt zu zeigen:

$$
\bar{M}_{n}=\underline{M}_{n+1}, n=1, \ldots, N-1,
$$

d.h. die Intervalle überdecken den Bereich $\left(0, \bar{M}_{N}\right]$.

Benutzt man die Identität

$$
\text { (5.1.11) } \begin{aligned}
\underline{M}_{n} & =a\left(\bar{K}_{n} \sum_{i=1}^{n} E_{i} b_{i}-\sum_{i=1}^{n} K_{i}\right) \\
& =a\left(\bar{K}_{n} \sum_{i=1}^{n-1} E_{i} b_{i}-\sum_{i=1}^{n-1} K_{i}\right) \text { für alle } n \in \mathbf{N},
\end{aligned}
$$

so sieht man sofort:

$$
\underline{M}_{n+1}=\bar{M}_{n}=a\left(\bar{K}_{n+1} \sum_{i=1}^{n} E_{i} b_{i}-\sum_{i=1}^{n} k_{i}\right)
$$




\section{Stetigkeit:}

Klar ist, in den offenen Intervallen $M \in\left(\underline{M}_{n}, \bar{M}_{n}\right), n=1, \ldots, N$ ist $G(M)$ stetig. Bleibt $z u$ zeigen: $G(M)$ ist stetig an allen Intervallgrenzen

$$
\bar{M}_{n}=\underline{M}_{n+1}, n=1, \ldots, N-1,
$$

d.h. es muß gelten fur $n=1, \ldots, N-1$ mit $h>0$

$$
G\left(\bar{M}_{n}\right)=\lim _{h \rightarrow 0} G\left(\underline{M}_{n+1}+h\right)
$$

Man erhält durch Einsetzen von $(5.1 .10)$ in $(5.1 .8)$

$$
(5.1 .12) \quad G\left(\bar{M}_{n}\right)=\bar{K}_{n+1}
$$

und durch Einsetzen von (5.1.9) in (5.1.8)

$$
\lim _{h \rightarrow 0} G\left(\underline{M}_{n+1}+h\right)=\bar{K}_{n+1} \text {. }
$$

\section{Strenge Monotonie:}

In den offenen Intervalien

$$
M \in\left(\underline{M}_{n}, \bar{M}_{n}\right), \quad n=1, \ldots, N
$$

ist die Steigung

$$
\text { (5.1.13) } \frac{d G(M)}{d M}=\frac{1}{a \sum_{i=1}^{n} E_{i} b_{i}}>0 .
$$

Zusammen mit der stetigkeit folgt daraus die strenge Monotonie auf dem ganzen Bereich $\left(0, \overline{\mathrm{M}}_{\mathrm{N}}\right]$.

$$
\text { D }
$$


Die Funktion $\mathrm{G}(\mathrm{M})$ besteht aus $\mathrm{N}$ Geradenabschnitten. An den Intervallgrenzen hat sie jeweils einen "Knick", weil stets

$(5.1 .14)$

$$
\frac{1}{a \sum_{i=1}^{n} E_{i} b_{i}}>\frac{1}{a \sum_{i=1}^{n+1} E_{i} b_{i}} \Leftrightarrow E_{n+1} b_{n+1}>0
$$

gilt. Die Steigung ist in den einzelnen Intervallen konstant positiv, sie nimmt ab, wenn $M$ eine Intervallgrenze überschreitet. Gleichzeitig steigt die Zahl der zuweisungsberechtigten Gemeinden.

\section{Exkurs: Verfahren (4) für die Berechnung des Grund-} betrags

Das Verfahren beruht auf der Idee, das Intervall zu bestimmen, in dem eine gegebene Schlüsselmasse liegt. Damit ist gleichzeitig der zugehörige Grundbetrag gefunden.

\section{Algorithmus 4}

(O) Anfangsgrößen einlesen: $M, a, K_{1}, E_{1}, b_{1}$

(1) Initialisierung $n:=1$

(2) Repeat

$$
\begin{aligned}
& \text { Werte } K_{n+1}, E_{n+1}, b_{n+1} \text { einlesen } \\
& \bar{M}_{n} \text { berechnen } \\
& n:=n+1
\end{aligned}
$$

until $M \leqq \bar{M}_{n}$ 
(3) Richtiges Intervall gefunden:

Berechne

$$
G=\frac{\frac{1}{a} M+\sum_{i=1}^{n} K_{i}}{\sum_{i=1}^{n} E_{i} b_{i}}
$$

und ausdrucken.

Ende.

Der Algorithmus prüft also sukzessiv, beginnend mit dem ersten Intervall, ob die Schlüsselmasse in diesem Intervall liegt. Wenn ja, ist der zugehörige Grundbetrag gefunden und das Verfahren bricht ab. Nehmen wir an, daß $M$ eine stetige Zufallsvariable und auf $\left(0, \bar{M}_{N}\right]$ gleichverteilt sei, dann müssen im Mjttel $\frac{N}{2}$ Intervalle (N sei gerade) überprüft werden.

Dieser Aufwand läbt sich u.U. noch reduzieren, wenn man nicht bei $\mathrm{n}=1$, sondern mit der $\mathrm{zahl}$ der zuweisungsberechtigten Gemeinden aus dem Vorjahr beginnt.

\subsubsection{Die Schlüsselzuweisungen als Funktion der Schlüssel-} masse

Wir kehren nun zurück zur Spezifikation der Funktionen $S_{i}(M)$ und fragen dazu zunächst nach der Schlüsselmasse $M_{i}^{\circ}$, für die

$$
G\left(M_{i}^{\circ}\right)=\bar{K}_{i}
$$

ist. Wir erhalten bekanntlich (siehe 5.1.1) 
(5.1.15) $\quad M_{i}^{O}=\underline{M}_{i}=a\left(\bar{K}_{i} \sum_{j=1}^{i} E_{j} b_{j}-\sum_{j=1}^{i} K_{j}\right)$,

also genau die Schlüsselmasse, bei der die Gemeinde $i$ - 1 gerade noch positive Zuweisungen erhält und die Gemeinde i noch keine erhält.

Aufgrund der strengen Monotonie von $G(M)$ auf dem ganzen Definitionsbereich gilt weiterhin:

$$
\begin{aligned}
& G(M) \leqq \bar{K}_{i} \quad \text { für alle } \quad M \leqq \underline{M}_{i} \\
& G(M)>\bar{K}_{i} \text { für alle } M>\underline{M}_{i} \text {. }
\end{aligned}
$$

Daraus folgt

$(5 \cdot 1 \cdot 16)$

$$
\Theta_{i}(M)=\left\{\begin{array}{cc}
0, & M \leqq M_{i} \\
1, & M>\underline{M}_{i}
\end{array}\right.
$$

und

$$
\text { (5.1.17) } S_{i}(M)= \begin{cases}0 & 0<M \leqq M_{i} \\ a\left(E_{i} b_{i} G(M)-K_{i}\right), & M>\underline{M}_{i} .\end{cases}
$$

Da G(M) auf dem ganzen Definitionsbereich, insbesondere also auch für alle $M>\underline{M}_{i}$ definiert ist, ist auch $S_{i}(M)$ für alle $M>0$ definiert. Weiterhin wissen wir aufgrund der stetigkeit von $G(M)$, daß auch $S_{i}(M)$ für alle $M>\underline{M}_{i}$ stetig ist. Für die Stetigkeit von $S_{i}$ auf dem ganzen Definitionsbereich bleibt zu zeigen: $S_{i}$ ist auch an der stelle $\underline{M}_{i}$ stetig.

Es gilt mit $h>0$ und mit $\lim _{M \rightarrow M_{i}+h} G(M)=\bar{K}_{i}$ : $\lim _{M \rightarrow \underline{M}_{i}+h} S_{i}(M)=0=S_{i}\left(\underline{M}_{i}\right)$. 
Nun setzen wir noch $(5.1 .8)$ in $(5.1 .17)$ ein und erhalten die abschnittsweise definierten Funktionen

$(5.1 .18)$

$$
S_{i}(M)= \begin{cases}0,0<M \leqq M_{i} \\ \frac{E_{i} b_{i}}{\sum_{j=1}^{n} E_{j} b_{j}}+a\left(\frac{\sum_{j=1}^{n} K_{j}}{\sum_{j=1}^{n} E_{j} b_{j}}-k_{i}\right), & M \in\left(M_{-n}, \bar{M}_{n}\right], \\ & n=i, \ldots, N .\end{cases}
$$

Fassen wir zusammen: Eine Gemeinde $i$ erhält also keine positive Schlüsselzuweisung, wenn die Schlüsselmasse eine bestimmte (gemeindeabhängige) Grenze nicht überschreitet. Diese Grenze ist umso höher, je größer die Steuerkraft pro modifiziertem Einwohner einer Gemeinde ist (weil bekanntlich $\underline{M}_{i}<\underline{M}_{i+1} \Leftrightarrow \bar{K}_{i}<\bar{K}_{i+1}$ gilt). Jenseits dieser Grenze wächst $s_{i}$ innerhalb $\left(\underline{M}_{n}, \bar{M}_{n}\right), n=i, \ldots, N$ linear mit $M$ mit der Steigung

$$
\text { (5.1.19) } \frac{d s_{i}(M)}{d M}=\frac{E_{i} b_{i}}{\sum_{j=1}^{n} E_{j} b_{j}} .
$$

Die Zuwächse von $S_{i}$ werden aber immer geringer, wenn $M$ Intervallgrenzen überschreitet, weil für $n=i, \ldots, N-1$

$$
\frac{E_{i} b_{i}}{\sum_{j=1}^{n} E_{j} b_{j}}>\frac{E_{i} b_{i}}{\sum_{j=1}^{n+1} E_{j} b_{j}} \leftrightarrow E_{n+1} b_{n+1}>0
$$

immer erfüllt ist. 


\subsection{Optimale Schlüsselmassen für Kommunen}

\subsubsection{Die Maximierung der Finanzausstattung}

Wir interessieren uns nun für eine Verteilung

$$
s\left(M_{i}^{*}\right)=\left(s_{1}\left(M_{i}^{*}\right), \ldots, s_{i}\left(M_{i}^{*}\right), \ldots, s_{N}\left(M_{i}^{*}\right)\right),
$$

bei der die Gemeinde $i$ ihre Finanzausstattung

$$
\text { (5.2.1) } \quad F_{i}(M):=S_{i}(M)+K_{i}
$$

maximiert. Eine Schlüsselmasse $M_{i}^{*} \in\left(0, \bar{M}_{N}\right]$ ist also optimal für $i$, wenn

$$
(5.2 .2) \quad F_{i}\left(M_{i}^{*}\right)=\max _{M} F_{i}(M)
$$

gilt. Mit der Äquivalenz

(5.2.3) $\quad \max _{M} F_{i}(M) \rightarrow \max _{M} S_{i}(M)$

maximieren die Gemeinden ihre Finanzausstattung durch Maximierung ihrer Schlüsselzuweisung.

Da $S_{i}(M)$ für alle $M>\underline{M}_{i}$ eine streng monoton wachsende Funktion von $M$ ist, wählen die Gemeinden die Obergrenze des Definitionsbereichs von $s_{i}(M)$

$$
\text { (5.2.4) } \quad M_{i}^{*}=\bar{M}_{N} \quad, \quad i=1, \ldots, N \text {. }
$$

Wird für $M$ exogen eine Restriktion $\bar{M}<\bar{M}_{N}$ festgesetzt (was schon allein aufgrund der Beschränkung der öffentlichen Haushalte von der Einnahmenseite her plausibel ist), dann ist die Lösung des beschränkten Optimierungsproblems 
$(5.2 .5)\left\{\begin{array}{l}\max _{M} S_{i}(M) \\ M \leqq \bar{M}\end{array}\right.$

durch

$$
\text { (5.2.6) } \quad M_{i}^{*}= \begin{cases}\{\bar{M}\}, & \bar{M}>\underline{M}_{i}^{1} \\ (0, \bar{M}], & \bar{M} \leqq \underline{M}_{i}\end{cases}
$$

gegeben. Die maximale Finanzausstattung ist

$$
F_{i}\left(M_{i}^{*}\right)= \begin{cases}s_{i}(\bar{M})+k_{i}, & \bar{M}>\underline{M}_{i} \\ k_{i} & , \bar{M} \leqq \underline{M}_{i}\end{cases}
$$

\subsubsection{Maximierung des Finanzanteils}

Im Gegensatz zum vorigen Abschnitt, in dem eine Gemeinde nur an ihrer absoluten Position interessiert war, nehmen wir nun an, sie habe das ziel, ihren Finanzanteil

(5.2.7) $\quad A_{i}(M):=\frac{F_{i}(M)}{\sum_{j=1}^{N} F_{j}(M)}=\frac{s_{i}(M)+K_{i}}{K+M}$

zu maximieren. Eine Schlüsselmasse $\mathrm{M}_{i}^{*}$ ist demnach optimal für $i$, wenn

(5.2.8) $\quad A_{i}\left(M_{i}^{*}\right)=\max _{M} A_{i}(M)$

1) $\mathbb{M}_{i}^{*}=\left\{M_{i}^{*}\right\}$ 
ist. Damit ist die Gemeinde auch relativ in der günstigsten Position, denn mit

(5.2.9) $\quad \sum_{i=1}^{N} A_{i}(M)=1$ für alle $M$

ist der Finanzanteil von i nur dann maximal, wenn der gesamte Finanzanteil aller anderen Gemeinden minimal ist.

Untersuchen wir $A_{i}(M)$. Aufgrund der Stetigkeit von $S_{i}(M)$ auf dem Definitionsbereich $\left(0, \bar{M}_{N}\right]$ ist $A_{i}(M)$ für alle $M \in\left(0, \bar{M}_{N}\right]$ definiert und stetig.

Wir werden sehen, daß es für die Maximierung des Finanzanteils einer Gemeinde auch sinnvoll sein kann, Schlüsselmassen von Null zu wählen, d.h. es kann aus der Sicht einer einzelnen Gemeinde optimal sein, wenn überhaupt keine $\mathrm{zu}-$ weisungen an Gemeinden verteilt werden. Da aber $S_{i}(M)$ nur für positive Schlüsselmassen definiert ist, vereinbaren wir eine stetige Erweiterung $^{1)}$ des Definitionsbereichs

$(5.2,10) \quad s_{i}(0):=0$ für alle $i=1, \ldots, N$.

Dies ist mit dem System der Schlüsselzuweisungen durchaus verträglich, denn damit gilt

$$
\sum_{i=1}^{N} S_{i}(M)=M \quad \text { für } M=0 \text {. }
$$

1) Eine Schlüsselmasse von Null haben wir ursprünglich nur deshalb nicht zugelassen, weil der Grundbetrag dafür nicht definiert war. So mußten wir davon ausgehen, daß mindestens eine Gemeinde positive Schlüsselzuweisungen erhält, was bei einer positiven Schlüsselmasse bekanntlich immer der Fall ist. 
Setzt man (5.1.8) und (5.2.10) in (5.2.7) ein, erhält man $(5.2 .11)$

$$
A_{i}(M)= \begin{cases}\frac{K_{i}}{M+K}, & 0 \leqq M \leqq M_{i} \\ \left(\frac{E_{i} b_{i}}{\sum_{j=1}^{n} E_{j} b_{j}}+\frac{a E_{i} b_{i} \sum_{j=1}^{n} K_{j}}{\sum_{j=1}^{n} E_{j} b_{j}}+(1-a) K_{i}\right) \frac{1}{M+K}, & M \in\left(\underline{M}_{n}, \bar{M}_{n}\right], \\ n=i, \ldots, N .\end{cases}
$$

W1e man leicht sieht, ist $A_{i}(M)$ im Bereich $\left[0, M_{i}\right]$ streng monoton fallend und

$$
(5.2 .12) \quad \mathrm{LM}_{i}^{*}=0
$$

ist das lokale Maximum in diesem Bereich.

Um den Verlauf der Funktion für $M>\underline{M}_{i}$ beurteilen zu können, berechnen wir in den (offenen) Intervallen $\left(\underline{M}_{n}, \bar{M}_{n}\right)$, $\mathrm{n}=\mathrm{i}, \ldots, \mathrm{N}$ die steigungen

$(5.2 .13)$

$$
\frac{d A_{i}(M)}{d M}=\frac{\frac{E_{i} b_{i}}{\sum_{j=1}^{n} E_{j} b_{j}}\left(k-a \sum_{j=1}^{n} K_{j}\right)-(1-a) k_{i}}{(M+K)^{2}} .
$$

Innerhalb eines Intervalls ist die steigung entweder positiv, negativ oder gleich Null. Da der Nenner immer positiv ist, hängt das Vorzeichen der Ableitung vom Zähler ab. Der Nenner wächst streng monoton mit $M$. Der Zähler wird mit wachsendem $M$ kleiner, wenn $M$ Intervallgrenzen überschreitet. Zur Bestimmung von $\mathrm{M}_{i}^{*}$ können wir also so vorgehen: 
a) Falls für $M \in\left(\underline{M}_{i}, \bar{M}_{i}\right)$

(5.2.14) $\quad \frac{\mathrm{dA}_{i}(\mathrm{M})}{\mathrm{dM}} \leqq 0$

gilt, dann ist auch

$$
\frac{d A_{i}(M)}{d M}<0 \quad \text { für } M \in\left(\underline{M}_{n}, \bar{M}_{n}\right), \quad n=i+1, \ldots, N
$$

Daraus folgt zusammen mit der stetigkeit, daB $A_{i}(M)$ auf ganz $\left[0, \overline{\mathrm{M}}_{\mathrm{N}}\right]$ monoton fallt. Mit der strengen Monotonie auf $\left[0, \underline{M}_{i}\right]$ folgt

(5.2.15) $\quad M_{i}^{*}=\mathrm{LM}_{i}^{*}=0$.

Die Bedingung $(5.2 .14)$ ist äquivalent mit

$(5.2 .16)$

$$
\frac{k-a \sum_{j=1}^{i} K_{j}}{(1-a) \sum_{j=1}^{i} E_{j} b_{j}} \leqq \bar{K}_{i} .
$$

Sie wird zumindest von der Gemeinde $N$ erfüllt, denn für $i=\mathrm{N}$ verlangt $(5.2 .16)$

$$
\frac{\sum_{j=1}^{N} K_{j}}{\sum_{j=1}^{N} E_{j} b_{j}} \leqq \bar{K}_{N},
$$

was für alle $N \geqq 1$ gilt. 
b) Falls für $M \in\left(\underline{M}_{i}, \bar{M}_{i}\right)$

$$
\frac{d A_{i}(M)}{d M}>0
$$

dann gibt es ein lokales Maximum

(5.2.17) $\quad \mathrm{RM}_{\mathrm{i}}^{*}=\underline{M}_{\mathrm{n}} * \overline{\mathrm{M}}_{\mathrm{n} *-1}$

im Bereich $\left(\underline{M}_{i}, \bar{M}_{N}\right]$, wenn ein $n * \in\{1+1, \ldots, N\}$ existiert mit

$$
\frac{d A_{i}(M)}{d M}>0, \quad M \in\left(\underline{M}_{n *-1}, \bar{M}_{n *-1}\right)
$$

und

$$
\frac{d A_{i}(M)}{d M}<0 \quad, \quad M \in\left(\underline{M}_{n *}, \bar{M}_{n *}\right)
$$

Dies ist genau dann der Fall, wenn für $n^{*}$

$(5.2 .18)$

$$
\frac{k-a \sum_{j=1}^{n *-1} k_{j}}{(1-a) \sum_{j=1}^{n *-1} E_{j} b_{j}}>\bar{k}_{i}>\frac{k-a \sum_{j=1}^{n^{*}} k_{j}}{(1-a) \sum_{j=1}^{n^{*}} E_{j} b_{j}}
$$

erfüllt ist. Das lokale Maximum

$$
\text { (5.2.19) } \quad \mathrm{RM}_{\mathrm{i}}^{*}=\overline{\mathrm{M}}_{\mathrm{N}}
$$

liegt am rechten Rand des Definitionsbereichs (Randmaximum), wenn die Steigung im letzten Intervall noch positiv ist, d.h. falls 


$$
\frac{d A_{i}(M)}{d M}>0, M \in\left(\underline{M}_{N}, \bar{M}_{N}\right) \text {, }
$$

und damit

(5.2.20) $\quad \bar{k}_{i}<\frac{\sum_{j=1}^{N} K_{j}}{\sum_{j=1}^{N} E_{j} b_{j}}$

erfüllt ist.

c) Falls ein $n * \in\{i+1, \ldots, N\}$ existiert mit

$$
\frac{d A_{1}(M)}{d M}=0 \text { für } M \in\left(M_{n^{*}}, \bar{M}_{n^{*}}\right) \text {, }
$$

d.h. falls

$$
(5.2 .21) \quad \bar{k}_{i}=\frac{k-a \sum_{j=1}^{n *} k_{j}}{(1-a) \sum_{j=1}^{n^{*}} E_{j} b_{j}}
$$

gilt ${ }^{1)}$, dann ist die Menge der optimalen lokalen Schlüsselmassen $R M_{1}^{*}=\left\{R M_{i}^{*}\right\}$ das Intervall

(5.2.22) $\quad R M_{1}^{*}=\left[\underline{M}_{n} *, \bar{M}_{n *}\right]$.

Um in den Fallen b) und c) das globale Optimum zu finden, müssen wir die lokalen Optima vergleichen, wobei noch vereinfachend

$$
\mathrm{RM}_{\mathrm{i}}^{*}=\mathrm{RM}_{\mathrm{i}}^{*}=\underline{\mathrm{M}}_{\mathrm{n}}{ }^{*}
$$

angenommen wird.

1) Die Bedingung (5.2.21) wird sicher nur zufällig erfüllt sein, da die rechte Seite nur (N-i) diskrete Werte annehmen kann. 
Allgemein gilt:

$$
\begin{aligned}
& M_{i}^{*}= \begin{cases}R_{i}^{*} & A_{i}\left(R M_{i}^{*}\right)>A_{i}\left(L M_{i}^{*}\right) \\
L M_{i}^{*} & A_{i}\left(L M_{i}^{*}\right)>A_{i}\left(R M_{i}^{*}\right)\end{cases} \\
& M_{i}^{*}=\left\{R M_{i}, L M_{i}\right\} \oplus A_{i}\left(L M_{i}^{*}\right)=A_{i}\left(R M_{i}^{*}\right) .
\end{aligned}
$$

Wir wissen

$$
A_{i}^{*}\left(L M_{i}^{*}\right)=\frac{K_{i}}{K}
$$

Für $R M_{i}^{*}=M_{n} * n * \in\{i+1, \ldots, N\}$ gilt

$$
s_{i}\left(R M_{i}^{*}\right)=a E_{i} b_{i} G\left(\underline{M}_{n}^{*}\right)-a K_{1}=a\left(E_{1} b_{i} \bar{K}_{n *}-K_{i}\right)
$$

und

$$
A_{i}\left(R M_{i}^{*}\right)=\frac{a E_{1} b_{i} \bar{K}_{n *}+(1-a) K_{i}}{\underline{M}_{n^{*}}+K} .
$$

Damit folgt:

$(5.2 .23) \quad \mathrm{A}_{i}\left(\mathrm{LM}_{i}^{*}\right)<\mathrm{A}_{i}\left(\mathrm{RM}_{i}^{*}\right)-\overline{\mathrm{K}}_{i}<\overline{\mathrm{K}}_{\mathrm{n}^{*}}\left(\frac{\mathrm{aK}}{\mathrm{M}_{-\mathrm{n}^{*}}+\mathrm{aK}}\right)$.

Für $\mathrm{RM}_{i}^{*}=\bar{M}_{\mathrm{N}}($ Randmaximum) ist

$$
A_{i}\left(\bar{M}_{N}\right)=\frac{E_{i} b_{i}}{\sum_{j=1}^{N} E_{j} b_{j}}-\frac{a K_{i}}{\bar{M}_{N}+k} .
$$

Falls $\overline{\mathrm{M}}_{\mathrm{N}} \rightarrow \infty$, dann folgt

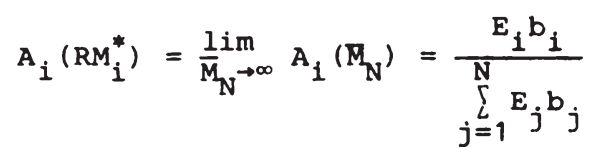


und

(5.2.24) $\quad A_{i}\left(L_{i}^{*}\right)<A_{i}\left(R_{i}^{*}\right) \leftrightarrow \bar{K}_{i}<\frac{K}{\sum_{j=1}^{N} E_{j} b_{j}}$

Wir nehmen im folgenden wieder an, daß $M$ exogen auf $\bar{M}<\bar{M}_{N}$ beschränkt sei. Eine Gemeinde sehe sich also dem beschränkten Optimierungsproblem

$(5.2 .25)\left\{\begin{array}{l}\max A_{i}(M) \\ M \leqq \bar{M}\end{array}\right.$

gegenüber. Ein beschränktes $\bar{M}_{i}^{*} \in(0, \bar{M}]$ ist optimal, falls es Lösung von $(5.2 .25)$ ist, d.h. es muß gelten:

$$
A_{i}\left(\bar{M}_{i}^{*}\right)=\max _{M \in(O, \bar{M}]} A_{i}(M)
$$

Wir unterscheiden zwei Fälle:

a) $\bar{M} \leqq \underline{M}_{i} \cdot$ Dann ist

(5.2.26) $\quad \bar{M}_{i}^{*}=L M_{i}^{*}=0$.

b) $\bar{M}>\underline{M}_{i}$. Falls zusätzlich $M_{i}^{*} \leqq \bar{M}$ gilt, dann ist

(5.2.27) $\quad \bar{M}_{i}^{*}=M_{i}^{*}$.

Falls $\bar{M}>\underline{M}_{i}$ und zusätzlich $\bar{M}<M_{i}^{*}$ ist, dann gibt es ein beschränktes lokales Maximum $\overline{\mathrm{RM}}_{i}^{*}$ im Bereich $\left(\underline{M}_{i}, \overline{\mathrm{M}}\right]$ mit

$$
A_{i}\left(\overline{R M}_{i}^{*}\right)=\max _{M \in\left(\underline{M}_{i}, \bar{M}\right]} A_{i}(M)
$$

Da es im Bereich $\left(\underline{M}_{i}, \bar{M}_{N}\right)$ nur ein unbeschränktes lokales 
Maximum $\mathrm{RM}_{i}^{*}$ gibt und weil mit der Voraussetzung $M_{i}^{*}>\bar{M}>\underline{M}_{i}$ das unbeschränkte globale Maximum $M_{i}^{*}=\mathrm{RM}_{i}^{*}$ ist, folgt für das beschränkte lokale Maximum

$$
\overline{\mathrm{RM}}_{i}^{*}=\overline{\mathrm{M}}
$$

Das beschränkte globale Maximum erhält man als

$$
\begin{aligned}
& \bar{M}_{i}^{*}=\left\{\begin{array}{cc}
\overline{R M}_{i}^{*}=\bar{M} & \Leftrightarrow A_{i}(\bar{M})>\frac{K_{i}}{K}=A_{i}\left(L M_{i}^{*}\right) \\
0 & \leftrightarrow A_{i}(\bar{M})<\frac{K_{i}}{K}
\end{array}\right. \\
& \overline{\mathbb{M}}_{i}^{*}=\{0, \bar{M}\} \Leftrightarrow A_{i}(\bar{M})=\frac{K_{i}}{K} .
\end{aligned}
$$

Aus den Optimalbedingungen kann man eigentlich nur für sehr arme und sehr reiche Gemeinden Aussagen über die Höhe der optimalen Schlüsselmasse ableiten. Sehr reiche Gemeinden, die sicher (5.2.16) erfüllen, präferieren eine Schlüsselmasse $M_{i}^{*}=0$. Sehr arme Gemeinden erfüllen die Bedingung (5.2.20) und (5.2.23) und wünschen sich den rechten Rand des Definitionsbereichs $M_{i}^{*}=\bar{M}_{N}$ als Schlüsselmasse. Dieses Ergebnis ist für arme Gemeinden aber sehr ernüchternd, denn die Schlüsselmasse ist in allen praktisch relevanter Fällen auf einen Betrag $\bar{M}$ beschränkt, der sehr viel niedriger als $\bar{M}_{N}$ sein dürfte, so daß sich arme Gemeinden praktisch nie im (unbeschränkten) Optimum befinden. Bei reichen Gemeinden kann natürlich eine Beschränkung nichts an ihrer optimalen Schlüsselmasse ändern, allerdings werden sie sich praktisch auch nie im Optimum befinden, weil die tatsächlichen Schlüsselmassen in der Regel erheblich größer sind. So ist zu vermuten, daß bei den realisierten Schlüsselmassen am ehesten Gemeinden mit mittlerer normierter steuerkraft ihrem Optimum relativ nahe kommen. 


\subsection{Die Variation der Schlüsselmasse aus Sicht von Gemeindegruppen}

Wir gehen nachfolgend von einer beliebigen, aber fixen Schlüsselmasse aus. Das könnte etwa die Schlüsselmasse sein, die in einem bestimmten Jahr tatsächlich für die Kommunen zur Verfügung steht. Wir fragen dann nach der Veränderung der Finanzausstattung bzw. des Finanzanteils von Gemeinden und Gemeindegruppen, wenn diese Schlüsselmasse um einen bestimmten Betrag erhöht oder verringert wird.

\subsubsection{Die Verteilung der Finanzausstattungen}

Nehmen wir eine fixe schlüsselmasse $M \in\left(\underline{M}_{n}, \bar{M}_{n}\right]$ mit $n \in\{1, \ldots, N\}$ belleblg an. Dann ist der zugehörige Grundbetrag durch

$(5.3 .1)$

$$
G(M)=\frac{\frac{M}{a}+\sum_{i=1}^{n} K_{i}}{\sum_{i=1}^{n} E_{i} b_{i}}
$$

bestimmt, und $\mathrm{n}$ ist die Zahl der zuweisungsberechtigten Gemeinden (siehe $(5,1.8)$ ). Nun verändere sich $M$ um $\Delta M$, wobei noch die Restriktion

$$
\text { (5.3.2) } \quad 0<\Delta M+M \leqq \bar{M}_{N} \quad \Rightarrow \quad \Delta M \in\left(-M, \bar{M}_{N}-M\right]
$$

zu beachten ist.

Die Änderung der Schlüsselzuweisung einer Gemeinde i in Abhängigkeit von $\Delta M$ sei durch

$$
\text { (5.3.3) } \quad \Delta S_{i}(\Delta M):=S_{i}(M+\Delta M)-S_{i}(M)
$$


und die Änderung der Finanzausstattung einer Gemeinde i in Abhängigkeit von $\Delta M$ durch

$$
(5.3 .4) \quad \Delta F_{i}(\Delta M):=F_{i}(M+\Delta M)-F_{i}(M)
$$

definiert. Mit der Definitionsgleichung (5.2.1) folgt sofort

$$
(5.3 .5) \quad \Delta F_{i}(\Delta M)=\Delta S_{i}(\Delta M) \text {. }
$$

Die Anderung der Finanzausstattung ist also allein auf die Veränderung der Schlüsselzuweisung zurückzuführen, was wegen der konstanten steuerkraft auch einsichtig ist.

Wir wissen, allgemein ist die Funktion $S_{i}(M)$ durch

$(5.1 .27) \quad S_{i}(M)= \begin{cases}0 & M \leqq M_{i} \\ a\left(E_{i} b_{i} G(M)-k_{i}\right), & M>\underline{M}_{i}\end{cases}$

spezifiziert (siehe 5.1). Zur Berechnung der Funktion $\Delta S_{i}(\Delta M)$ müssen wir daher unterscheiden, ob bei der Schlüsselmasse $M$ die Gemeinde $i$ Schlüsselzuweisungen erhalten hat (Fall b) oder nicht (Fall a).

Fall a) $\quad M \leqq \underline{M}_{i}$, d.h. $M \in\left(\underline{M}_{n}, \bar{M}_{n}\right], n \in\{1, \ldots, i-1\}$.

Man erhält

(5.3.6) $\Delta S_{i}(\Delta M)= \begin{cases}0 & , \Delta M \leqq M_{i}-M . \\ a\left(E_{i} b_{i} G(M+\Delta M)-K_{i}\right), & , \Delta M>\underline{M}_{i}-M .\end{cases}$

Falls also $\Delta M$ so gro $B$ ist, daB die Gemeinde $i$ nachher Schlüsselzuweisungen erhält, dann ist natürlich 


$$
\Delta S_{i}(\Delta M)=S_{i}(M+\Delta M)
$$

Andernfalls ist $\Delta S_{1}(\Delta M)=0$, weil die Gemeinde weder vorher noch nachher zuweisungsberechtigt ist. In (5.3.6) ist $G(M+\Delta M)$ durch $(5.1 .8)$ spezifiziert.

$\underline{\text { Fall b) }} \quad M>\underline{M}_{i} \quad \rightarrow \quad \underline{M}_{i}-M<0$,

$$
\text { d.h. } M \in\left(\underline{M}_{n}, \bar{M}_{n}\right], n \in\{i, i+1, \ldots, N\} \text {. }
$$

Man erhält

(5.3.7) $\quad \Delta S_{i}(\Delta M)= \begin{cases}-a\left(E_{i} b_{i} G(M)-K_{i}\right), & \Delta M \leqq M_{i}-M \\ a E_{i} b_{i} \Delta G(\Delta M) & , \Delta M>\underline{M}_{i}-M .\end{cases}$

Dabei definieren wir

(5.3.8) $\quad \Delta G(\Delta M):=G(M+\Delta M)-G(M)$

als Änderung des Grundbetrags bei einer Änderung der Schlüsselmasse.

Falls die Schlüsselmasse so stark abnimmt, daß die Gemeinde $i$ aus dem Kreis zuweisungsberechtigter Gemeinden ausscheidet, dann entspricht der Rückgang der zuweisungen natürlich genau dem Betrag, den die Gemeinde vorher als Zuweisungen erhalten hatte. Falls die Schlüsselmasse nicht so stark abnimmt oder steigt, dann entspricht $\Delta S_{1^{\prime}}$ bezogen auf einen repräsentativen Einwohner, dem a-fachen der Änderung des Grundbetrags, die bei $\Delta M>0$ positiv und bei $\Delta M<0$ negativ ist.

Um in den Fällen a) und b) $\Delta S_{i}$ direkt von $\Delta M$ abhängig zu machen, müBte man in $(5.3 .6)$ und $(5.3 .7) \mathrm{G}(\mathrm{M}+\Delta \mathrm{M})$ und $\Delta G(\Delta M)$ durch ihre Funktionsgleichung und $G(M)$ durch den 
Funktionswert ersetzen. Da dies aber sehr aufwendig ist und für die weitere Analyse auch nicht benötigt wird, wollen wir darauf nicht näher eingehen.

Wir fragen nun, wie sich eine Erhöhung oder Verringerung der Schlüsselmasse bei verschiedenen Gruppen von Gemeinden auswirkt. Dabei werden diejenigen Gemeinden der gleichen Gruppe zugeordnet, die sich vorher und nachher in der gleichen Situation befinden. Es lassen sich so drei Gruppen unterscheiden:

Gruppe A besteht aus Gemeinden, die vorher und nachher zuweisungsberechtigt sind. In Gruppe B befinden sich Gemeinden, die aus dem Kreis zuweisungsberechtigter Gemeinden ausscheiden (bei Verringerung von $M$ ) oder neu hinzukommen (bei Erhöhung von $M$ ). In Gruppe $C$ sind alle Gemeinden, die weder vorher noch nachher zuweisungsberechtigt sind. Wir betrachten zuerst eine

\section{Erhöhung der Schlüsselmasse}

Zusätzlich zur fixen Schlüsselmasse M gehen wir im folgenden von einer bestimmten Erhöhung von $M$ um den Betrag

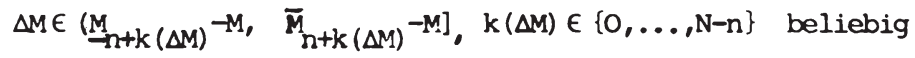

aus. Dann gibt $k:=k(\Delta M)$ in Abhängigkeit von $\Delta M$ die Zahl der Gemeinden an, die zum Kreis zuweisungsberechtigter Gemeinden neu hinzukommen, denn aufgrund der strengen Monotonie von $G(M)$ gilt

(5.3.9) $\Delta M>0 \Rightarrow k \geqq 0$. 


\section{A Gemeinden $\{1, \ldots, \mathrm{n}\}$}

Für sie gilt $\underline{M}_{i}<M<M+\Delta M$. Aus $(5.3 .7)$ entnimmt man

(5.3.10) $\Delta S_{i}(\Delta M)=a E_{i} b_{i} \Delta G(\Delta M)$.

B Gemeinden $\{n+1, \ldots, n+k\}$

Für sie gilt $M \leqq M_{i}<\Delta M+M$. Aus $(5.3 .6)$ ist zu entnehmen

(5.3.11) $\quad \Delta S_{i}(\Delta M)=a\left(E_{i} b_{i} G(M+\Delta M)-K_{i}\right)$.

Mit der Definitionsgleichung (5.3.8) folgt daraus

$$
\Delta S_{i}(\Delta M)=a\left(E_{i} b_{i} G(M)-K_{i}\right)+a E_{i} b_{i} \Delta G(\Delta M)<a E_{i} b_{i} \Delta G(\Delta M),
$$

da mit

$$
\begin{aligned}
& \underline{M}_{i} \geqq M \\
& G(M) \leqq \bar{K}_{i} \quad, \quad i=n+1, \ldots, n+k
\end{aligned}
$$

gilt.

C Gemeinden $\{n+k+1, \ldots, N\}$

Für sie gilt $\underline{M}_{i} \geqq M+\Delta M>M$. Aus $(5.3 .6)$ resultiert

(5.3.12) $\quad \Delta S_{i}(\Delta M)=0$.

Zusammenfassend kann man mit $\Delta S_{i}(\Delta M):=\frac{\Delta S_{i}(\Delta M)}{E_{i} b_{i}}$ schreiben:

$(5.3 .13) \Delta \bar{S}_{1}(\Delta M)= \begin{cases}Q & , i=n+k+1, \ldots, N \\ a\left(G(M)-\bar{K}_{i}\right)+a \Delta G(\Delta M), & i=n+1, \ldots, n+k \\ a \Delta G(\Delta M) & , i=1, \ldots, n .\end{cases}$ 
Der Anstieg der Schlüsselzuweisung eines repräsentativen Einwohners ist für alle Gemeinden aus der ärmsten Gruppe gleich groß, obwohl die Steuerkraftunterschiede in dieser Grupppe recht hoch sein können. Allerdings haben die Gemeinden aus der ärmsten Gruppe den gröBten Zuwachs, der noch größer ist als der Zuwachs der Gemeinden, die zum Kreis zuweisungsberechtigter Gemeinden neu hinzukommen. Die reichsten Gemeinden profitieren vom Anstieg der Schlüsselmasse überhaupt nicht.

\section{Verringerung der Schlüsselmasse}

Für den Fall, daß die Schlüsselmasse um den fixen Betrag

$$
\Delta M \in\left(M_{-n+k}(\Delta M)-M, \bar{M}_{n+k(\Delta M)}-M\right], k(\Delta M) \in\{1-n, \ldots, 0\} \text { beliebig }
$$

verringert wird, gibt $k:=k(\Delta M)$ die $\mathrm{Zahl}$ der Gemeinden an, die aus dem Kreis zuweisungsberechtigter Gemeinden ausscheiden, denn wegen der strengen Monotonie von G(M) gilt

$$
\Delta \mathrm{M}<\mathrm{O} \Rightarrow \mathrm{k} \leqq 0
$$

Ein $z u \quad \Delta M>0$ analoges Vorgehen liefert

$$
\text { (5.3.14) } \quad \Delta \bar{S}_{i}(\Delta M)= \begin{cases}0 & i=n+1, \ldots, N \\ -a\left(G(M)-\bar{K}_{i}\right), & i=n+k+1, \ldots, n \\ a \Delta G(\Delta M) \quad, \quad i=1, \ldots, n+k .\end{cases}
$$

Die Gruppe der reichsten Gemeinden verzeichnet bei sinkender Schlüsselmasse überhaupt keinen Rückgang ihrer normierten Finanzausstattung. Dagegen sinkt sie bei den ärmsten Gemeinden am stärksten, sogar noch stärker als bei den Gemeinden der mittleren Gruppe, weil mit $\overline{\mathrm{K}}_{\mathrm{i}}>\mathrm{G}(\mathrm{M}+\Delta \mathrm{M}), \mathrm{i}=\mathrm{n}+\mathrm{k}+1, \ldots, \mathrm{n}$ 


$$
-a\left(G(M)-\bar{K}_{i}\right)=-a\left(G(M+\Delta M)-\bar{K}_{i}\right)+a \Delta G(\Delta M)<a \Delta G(\Delta M)
$$

ist. Das ist recht überraschend, rein intuitiv würde man vielleicht vermuten, daß diejenigen Gemeinden die größten Einbußen hätten, die bei sinkender Schlüsselmasse aus dem. Krels der zuweisungsberechtigten Gemeinden ausscheiden. Ebenso könnte man sich leicht vorstellen, daß bei einer Erhöhung von M diejenigen Gemeinden die größten Zuwächse aufweisen, die zum Kreis der zuweisungsberechtigten Gemeinden neu hinzukommen. Beides ist aber nicht der Fall.

\subsubsection{Die Umverteilung der Finanzanteile}

Bei einer Erhöhung oder Verringerung der Schlüsselmasse sagt die Veränderung der Finanzausstattung einer Gemeinde noch nichts über ihre relative Position im Vergleich $z u$ anderen Gemeinden aus. Wir fragen deshalb nach der Veränderung des Finanzanteils einer Gemeinde, wenn sich die fixe Schlüsselmasse $M$ um $\Delta M$ ändert. Mit der Identität (5.2.7) und der Definitionsgleichung (5.3.3) ergibt sich allgemein für $i=1, \ldots, N$

$$
A_{i}(M+\Delta M)>A_{i}(M) \cdot\left\{\begin{array}{l}
\frac{\Delta S_{i}}{\Delta M}>A_{i}(M), \Delta M>0 \\
\frac{\Delta S_{i}}{\Delta M}<A_{i}(M), \Delta M<0 .
\end{array}\right.
$$

Das Verhältnis $\Delta \mathrm{S}_{i} / \Delta \mathrm{M}$ kann als Anteil der Gemeinde $i$ am $\mathrm{Zu}$ wachs (Rückgang) der Schlüsselmasse interpretiert werden. Eine Gemeinde hat demnach nach Erhöhung (Verringerung) von $M$ genau dann einen höheren Anteil als vorher, wenn ihr Anteil am Zuwachs (Rückgang) der Schlüsselmasse höher (niedriger) als ihr alter Finanzanteil ist. Entsprechend gilt 
(5.3.16) $\quad A_{i}(M+\Delta M)<A_{i}(M)-\left\{\begin{array}{l}\frac{\Delta S_{i}}{\Delta M}<A_{i}(M), \Delta M>0 \\ \frac{\Delta S_{i}}{\Delta M}>A_{i}(M), \Delta M<0 .\end{array}\right.$

Die Interpretation erfolgt analog $\mathrm{zu}$ (5.3.15).

Aus (5.3.15) und $(5.3 .16)$ folgt weiterhin für $\underline{\Delta M>0}$ :

$(5.3 .17) \quad A_{i}(M+\Delta M)\left\{\begin{array}{l}< \\ \}_{A}\end{array}\right.$

und für $\underline{\Delta M<0}$

$$
A_{i}(M+\Delta M)\left\{\begin{array} { l } 
{ < } \\
{ > }
\end{array} A _ { i } ( M ) \leftrightarrow \frac { \Delta S } { \Delta M } \left\{\begin{array}{l}
> \\
<
\end{array} A_{i}(M) .\right.\right.
$$

Wir betrachten zunächst eine Erhöhung der Schlüsselmasse $M$ um den fixen Betrag $\Delta M$, wobei $M$ und $\Delta M$ wie in 5.3 .1 spezifiziert seien. Dann lassen sich wieder drei Gruppen von Gemeinden unterscheiden:

C Gemeinden $i=n+k+1, \ldots, N$

Für sie gilt

(5.3.19) $\frac{\Delta S_{i}}{\Delta M}=0<A_{i}(M) \Rightarrow A_{i}(M+\Delta M)<A_{i}(M)$.

Reiche Gemeinden haben am Zuwachs der Finanzmasse keinen Anteil, ihr Finanzanteil nimmt daher ab. Gleichzeitig bedeutet dies, daß der Finanzanteil mindestens einer ärmeren Gemeinde zunimmt. 


\section{B Gemeinden $i=n+1, \ldots, n+k$}

Mit (5.3.13) und $F(M):=\sum_{i=1}^{N} F_{i}(M)$ ergibt sich

$(5.3 .20)$

$$
\frac{\Delta S_{i}}{\Delta M}>A_{i}(M) \leftrightarrow \frac{a\left(E_{i} b_{i} G(M)-K_{i}\right)+a E_{i} b_{i} \Delta G}{\Delta M}>\frac{K_{i}}{F(M)}
$$

$$
\omega \bar{K}_{i}<\frac{a F(M)}{\Delta M+a F(M)}(G(M)+\Delta G) \text {. }
$$

Da für alle $\Delta M>0$

$$
\frac{a F(M)}{\Delta M+a F(M)}(G(M)+\Delta G)<G(M)+\Delta G
$$

gilt, erhalten nicht in jedem Falle alle Gemeinden aus der Gruppe $\{n+1, \ldots, n+k\}$ einen höheren Finanzanteil als vorher. Notwendige Voraussetzung dafür, daß es überhaupt Gemeinden gibt, die (5.3.20) erfüllen, ist

$$
\begin{aligned}
& \frac{a F(M)}{a F(M)+\Delta M}(G(M)+\Delta G)>G(M) \\
& \rightarrow \frac{\Delta G}{G(M)} / \frac{\Delta M}{F(M)}>\frac{1}{a} .
\end{aligned}
$$

Um (5.3.21) zu interpretieren, definieren wir

$$
\text { (5.3.22) } \quad \Delta \mathrm{F}(\Delta \mathrm{M}):=\mathrm{F}(\mathrm{M}+\Delta \mathrm{M})-\mathrm{F}(\mathrm{M})
$$

als Veränderung der gesamten Finanzmittel.

Mit $F(M)=M+K$ folgt sofort

$$
(5.3 .23) \quad \Delta F:=\Delta F(\Delta M)=\Delta M \text {. }
$$


Damit läßt sich

$$
(5.3 .24) \quad \frac{\Delta G}{G(M)} / \frac{\Delta M}{F(M)}=\frac{\Delta G}{G(M)} / \frac{\Delta F}{F(M)}=: \varepsilon_{G, F}
$$

als Elastizität des Grundbetrags nach den gesamten Finanzmitteln interpretieren. Wir können diese auch in der Form

$$
(5.3 .25) \quad \varepsilon_{G, F}=\frac{\Delta G}{\Delta M} \cdot \frac{F(M)}{G(M)}
$$

schreiben. Man sieht, sie hängt von der Schlüsselmasse $M a b$ und von der stelgung $\Delta G / \Delta M$. Da wir von einem fixen Niveau $M$ ausgehen, wird $\varepsilon_{G, F}$ immer geringer, je größer $\Delta M$ ist, weil bekanntlich $\Delta \mathrm{G} / \Delta \mathrm{M}$ mit steigendem $\Delta \mathrm{M}$ kleiner wird (s. 5.1.1).

Mit (5.3.23) folgt für (5.3.21) der äquivalente Ausdruck

$$
(5.3 .26) \quad \varepsilon_{G, F}>\frac{1}{a} \text {. }
$$

Falls also

$$
(5.3 .27) \quad \varepsilon_{G, F} \leqq \frac{1}{a}
$$

gilt, gibt es in der Gruppe $\{n+1, \ldots, n+k\}$ keine Gemeinde, die nach Erhöhung der Schlüsselmasse einen höheren Finanzanteil als vorher hätte. 


\section{A Gemeinden $\{1, \ldots, n\}$}

Mit (5.3.13) gilt

$$
\begin{aligned}
& \frac{\Delta S_{i}}{\Delta M}>A_{i}(M) \quad-\frac{a E_{i} b_{i} \Delta G}{\Delta M}>\frac{a E_{i} b_{i} G(M)+(1-a) K_{i}}{M+K} \\
& \Leftrightarrow \vec{K}_{i}<\frac{a}{1-a}\left(F(M) \frac{\Delta G}{\Delta M}-G(M)\right) .
\end{aligned}
$$

Dabei ist

$$
\frac{a}{1-a}\left(F(M) \frac{\Delta G}{\Delta M}-G(M)\right) \leqq G(M) \rightarrow \varepsilon_{G, F} \leqq \frac{1}{a}
$$

(5.3.27) und (5.3.29) sind identisch, und deshalb werden, falls sie erfüllt sind, insgesamt gesehen nur Gemeinden aus der Gruppe $\{1, \ldots, n\}$ begünstigt, daraus aber bekanntlich mindestens eine Gemeinde.

Deshalb existiert ein $\hat{n} \in\{1, \ldots, n\}$, das durch

$$
\text { (5.3.30) } \quad \overline{\mathrm{K}}_{\mathrm{R}}<\frac{1}{1-\mathrm{a}}\left(\mathrm{F}(\mathrm{M}) \frac{\Delta \mathrm{G}}{\Delta \mathrm{M}}-\mathrm{G}(\mathrm{M})\right) \leqq \overline{\mathrm{K}}_{\hat{\mathrm{H}}+1}
$$

bestimmt ist und diejenige Gemeinde angibt, deren Finanzanteil sich gerade noch erhöht hat, d.h. es gilt

$$
(5.3 .31) \quad A_{i}(M+\Delta M)>A_{i}(M) \quad, \quad i=1, \ldots, \hat{n} .
$$

Falls (5.3.27) bzw. (5.3.29) nicht gelten, sondern (5.3.26) erfüllt ist, dann gilt in (5.3.29) statt " $\leqq$ " die Relation ">", und deshalb weisen alle Gemeinden $i=1, \ldots$, n nachher einen höheren Finanzanteil auf. Es können auch noch Gemeinden aus der Gruppe $\{n+1, \ldots, n+k\}$ profitieren. Falls also für ein $\tilde{n} \in\{n+1, \ldots, n+k\}$ 
$(5.3 .32)$

$$
\bar{K}_{\tilde{n}}<\frac{a F(M)}{\Delta M+a F(M)}(G(M)+\Delta G) \leqq \bar{K}_{\tilde{n}+1}
$$

gilt, dann ist

$$
A_{i}(M+\Delta M)>A_{i}(M), i=1, \ldots, \tilde{n} .
$$

Ansonsten ist

$$
A_{i}(M+\Delta M)>A_{i}(M) \quad, i=1, \ldots, n .
$$

Ob für ein bestimmtes $\Delta M$ die Bedingung (5.3.29) bzw. (5.3.27) oder die Bedingung (5.3.26) erfüllt ist, hängt natürlich von der Elastizität ab. Grob gesprochen kann man sagen, bei kleiner Elastizität werden weniger Gemeinden relativ begünstigt als bei großer Elastizität. Die Elastizität $\varepsilon_{G, F}$ ist eher klein, wenn die Erhöhung der Schlüsselmasse relativ hoch ausfällt. Dies ist etwas uberraschend, denn intuitiv würde man vermuten, daB sich mit einer merklichen Aufstockung der Schlüsselmasse auch relativ viele Gemeinden besserstellen lassen.

Wir betrachten nun eine Verringerung der Schlüsselmasse $M$ um einen bestimmten Betrag $\Delta M$, der wie in 5.3 .1 spezifiziert sei.

\section{Gemeinden $i=n+1, \ldots, N$}

Aus (5.3.14) ist zu entnehmen:

$$
\frac{\Delta S_{i}}{\Delta M}=0<A_{i}(M)=\frac{K_{i}}{M+K} \rightarrow A_{1}(M+\Delta M)>A_{i}(M) .
$$

Der Finanzanteil der reichsten Gemeinden nimmt $z u$. Das bedeutet gleichzeitig, daß der Finanzanteil mindestens einer ärmeren Gemeinde abnehmen muB. 


\section{B Gemeinden $1=n+k+1, \ldots, n$}

Für sie folgt mit (5.3.14):

$$
\frac{\Delta S_{i}}{\Delta M}>A_{i}(M)-\frac{-a\left(E_{i} b_{i} G(M)-K_{i}\right)}{\Delta M}>\frac{a E_{i} b_{i} G(M)+(1-a) K_{i}}{F(M)}
$$

$(5.3 .35)$

$$
\text { - } \bar{K}_{i}>G(M) \frac{a F(M+\Delta M)}{a F(M+\Delta M)-\Delta M} \text {. }
$$

Damit es überhaupt Gemeinden aus $\{n+k+1, \ldots, n\}$ gibt, die (5.3.35) erfüllen, mus wegen $\bar{K}_{i}<G(M)$ für $i=n+k+1, \ldots, n$ notwendigerweise

$$
G(M) \frac{a F(M+\Delta M)}{a F(M+\Delta M)-\Delta M}<G(M)
$$

sein, was für $\Delta M<0$ immer erfüllt ist.

Falls

$(5 \cdot 3 \cdot 37)$

$$
\begin{aligned}
& G(M) \frac{a F(M+\Delta M)}{a F(M+\Delta M)-\Delta M}<G(M)+\Delta G \\
& -\frac{1}{a} \geqq \frac{\Delta G}{\Delta M} \frac{F(M+\Delta M)}{G(M+\Delta M)}=\varepsilon_{G, F}(M+\Delta M)
\end{aligned}
$$

erfullt lst, dann profitieren sogar alle Gemeinden aus dieser Gruppe von einer Senkung der schlüsselmasse und

$$
A_{1}(M+\Delta M)>A_{1}(M) \text { für alle } i=n+k+1, \ldots, n \text {. }
$$

Dabei ist $\varepsilon_{G, F}(M+\Delta M)$ die Elastizität von $G$ nach $F$ bei der neuen Schlüsselmasse $M+\Delta M$. 


\section{A Gemeinden $i=1, \ldots, n+k$}

Mit $(5.3 .14) \mathrm{gilt}$

$$
\begin{aligned}
& \frac{\Delta S_{i}}{\Delta M}>A_{i}(M) \oplus \frac{a E_{i} b_{i} \Delta G}{\Delta M}>\frac{a E_{i} b_{i} G(M)+(1-a) K_{i}}{F(M)} \\
& \leftrightarrow \bar{K}_{i}<\frac{a}{1-a}\left(\frac{\Delta G}{\Delta M} F(M)-G(M)\right) .
\end{aligned}
$$

Dabei ist

$$
\frac{a}{1-a}\left(\frac{\Delta G}{\Delta M} F(M)-G(M)\right) \leqq G(M)+\Delta G \leftrightarrow \varepsilon_{G, F}(M+\Delta M) \leqq \frac{1}{a} .
$$

Die Bedingungen (5.3.37) und (5.3.39) sind identisch. Falls sie erfüllt sind, werden wegen $\bar{K}_{1}<G(M)+\Delta G, i=1, \ldots, n+k$ nur Gemeinden aus der Gruppe $\{1, \ldots, n+k\}$, also die ärmsten Gemeinden, schlechter gestellt, bekanntlich aber mindestens eine Gemeinde.

Daher existiert ein $\hat{n} \in\{1, \ldots, n+k\} m i t$

$$
\text { (5.3.40) } \quad \bar{R}_{\hat{n}}<\frac{a}{1-a}\left(\frac{\Delta G}{\Delta M} F(M)-G(M)\right) \leqq \bar{K}_{\hat{n}+1}
$$

und

$$
A_{i}(M+\Delta M)<A_{i}(M), i=1, \ldots, \hat{K} .
$$

Falls (5.3.37) bzw. (5.3.39) nicht erfült ist, sondern

$$
\varepsilon_{G, F}(M+\Delta M)>\frac{1}{a}
$$

gilt, dann ist auch

$$
\frac{a}{1-a}\left(\frac{\Delta G}{\Delta M} F(M)-G(M)\right)>G(M)+\Delta G \text {, }
$$


und daher

$$
A_{i}(M+\Delta M)<A_{i}(M) \text { für alle } i=1, \ldots, n+k \text {. }
$$

Es können sogar noch Gemeinden aus der Gruppe $\{n+k+1, \ldots, n\}$ schlechter gestellt sein, d.h. es kann ein $\tilde{n} \in\{n+k+1, \ldots, n\}$ mit

(5.3.43) $\quad \bar{K}_{\tilde{n}}<G(M) \frac{a F(M+\Delta M)}{a F(M+\Delta M)-\Delta M} \leqq \bar{K}_{\tilde{n}+1}$

existieren, so daß

(5.3.44) $\quad A_{i}(M+\Delta M)<A_{i}(M)$ für $i=1, \ldots, \tilde{n}$

gilt.

Bei Elastizitäten, die gröBer als 1/a sind, werden also vergleichsweise mehr Gemeinden schlechter gestellt als bei kleineren Elastizitäten. 


\subsection{Progressive und regressive Verteilungen}

Wir zeigen nun, daß von irgendzwei Verteilungen

$$
s:=s(M)=\left(s_{1}(M), \ldots, s_{N}(M)\right)
$$

und

$$
\hat{\mathbf{s}}:=s(\hat{M})=\left(s_{1}(\hat{M}), \ldots, s_{N}(\hat{M})\right)
$$

$\hat{\mathrm{s}}$ progressiver ist als $\mathrm{s}$ (also (3.2.11) erfüllt), wenn

$\hat{M}>M$ ist. Dazu mu $\beta \mathrm{xL}_{\mathrm{P}} Y$ gelten, wobei $x=\left(x_{1}, \ldots, x_{N}\right)$ bzw. $y=\left(y_{1}, \ldots, y_{N}\right)$ die Verteilung der normierten Finanzanteile bei $\hat{s}$ bzw. $s$ und

(5.4.1) $\quad x_{i}=\frac{\bar{F}_{i}(\hat{M})}{\sum_{j=1}^{N} \bar{F}_{j}(\hat{M})} \quad$ bzw. $\quad y_{i}=\frac{\bar{F}_{i}(M)}{\sum_{j=1}^{N} \bar{F}_{j}(M)}$

der normierte Finanzanteil von Gemeinde $i$ bei $s$ bzw. $\hat{s}$ ist.

$\mathrm{Zu}$ zeigen ist (siehe $(3.3 .6),(3.3 .7))$ :

a) $x_{1} \leqq x_{2} \leqq \cdots \leqq x_{N} \Rightarrow y_{1} \leqq y_{2} \leqq \cdots \leqq y_{N}$.

b) Es existiert ein $i_{0} \in\{1, \ldots, N-1\}$ mit

$$
\begin{aligned}
& y_{i} \leqq x_{i} \quad, \quad i=1, \ldots, i_{0} \\
& y_{i} \geqq x_{i}, \quad i=i_{0}+1, \ldots, N
\end{aligned}
$$

und zusätzlich

$$
\mathrm{y}_{1}<\mathrm{x}_{1} \wedge \mathrm{y}_{\mathrm{N}}>\mathrm{x}_{\mathrm{N}}
$$


ad a) Es gilt:

$$
\begin{aligned}
\left\{x_{1} \leqq x_{2} \leqq \cdots \leqq x_{N} \Rightarrow y_{1} \leqq y_{2} \leqq \cdots \leqq y_{N}\right\} \\
\left\{\begin{array}{l}
\bar{F}_{1}(\hat{M}) \leqq \bar{F}_{2}(\hat{M}) \leqq \cdots \leqq \bar{F}_{N}(\hat{M}) \\
\bar{F}_{1}(M) \leqq \bar{F}_{2}(M) \leqq \cdots \leqq \bar{F}_{N}(M)
\end{array}\right\}
\end{aligned}
$$

In 4.2 wurde gezeigt, daß für jede Verteilung der Schlüsselzuweisungen die Ordnung der normierten Finanzausstattungen die gleiche ist wie die ordnung der normierten steuerkraft.

$\underline{\text { ad b) }}$

Wir führen noch einige Bezeichnungen ein:

$$
\bar{F}(M):=\sum_{i=1}^{N} \vec{F}_{i}(M), M>0
$$

(5.4.2) $\quad \Delta \bar{S}_{i}:=\bar{S}_{i}(\hat{M})-\bar{S}_{1}(M)$,

$$
\Delta \bar{S}:=\sum_{i=1}^{N} \Delta \bar{S}_{1}
$$

Dann gilt allgemein:

(5.4.3) $\quad x_{i} \geqq y_{i} \leftrightarrow \frac{\bar{F}_{i}(\hat{M})}{\bar{F}(\hat{M})} \geqq \frac{\bar{F}_{i}(M)}{\bar{F}(M)} \cdot \frac{\Delta \bar{S}_{i}}{\Delta \bar{S}} \geqq \frac{\bar{F}_{i}(M)}{\bar{F}(M)}$.

Sei $\hat{M}:=\Delta M+M$ und seien $M$ und $\Delta M$ (fest) wie in 5.3 spezifiziert. Dann kann man (5.3.13) die Werte $\Delta \bar{S}_{i}:=\Delta \bar{S}_{i}(\Delta M)$ für $1=1, \ldots, \mathrm{N}$ entnehmen. 
Damit folgt für

$i=n+k+1, \ldots, N:$

(5.4.4) $\Delta \bar{S}_{i}=0 \Rightarrow \frac{\Delta \bar{S}_{i}}{\Delta \bar{S}}=0<\frac{\bar{K}_{i}}{\bar{F}(M)} \Rightarrow x_{i}<y_{i}$.

Damit gibt es wegen $\Sigma x_{i}=\Sigma y_{i}=1$ mindestens eine Gemeinde $i \operatorname{mit} x_{i}>y_{i}$.

$\underline{i}=\mathrm{n}+1, \ldots, \mathrm{n}+\mathrm{k}:$

(5.4.5) $\frac{\Delta \bar{S}_{i}}{\Delta \bar{S}}=\frac{a\left(G(\hat{M})-\bar{K}_{i}\right)}{\Delta \bar{S}}<\frac{\bar{K}_{i}}{\bar{F}(M)}=\frac{\bar{F}_{i}(M)}{\bar{F}(M)} * \bar{K}_{i}>\frac{G(\hat{M})}{1+\frac{\Delta \bar{S}}{a \bar{F}(M)}}$

$\mathrm{Da}$

$$
1+\frac{\Delta \bar{S}}{a \bar{F}(M)} \geqq 1 \wedge \quad \bar{K}_{i}>G(\hat{M}), \quad i=n+1, \ldots, n+k
$$

gilt, ist (5.4.5) erfüllt. Daraus folgt

$$
x_{i}<y_{i}, i=n+1, \ldots, n+k
$$

und damit gibt es unter den Gemeinden $\{1, \ldots, n\}$ mindestens ein $i \operatorname{mit} x_{i}>y_{i}$.

$\underline{i}=1, \ldots, \mathrm{n}:$

$(5.4 .6)$

$$
\begin{aligned}
& \frac{\Delta \bar{S}_{i}}{\Delta \bar{S}}>\frac{\bar{F}_{i}(M)}{\bar{F}(M)} \cdot \frac{a \Delta G}{\Delta \bar{S}}>\frac{a G(M)+(1-a) K_{i}}{\bar{F}(M)} \\
- & \bar{K}_{i}<\frac{a}{1-a}\left(\Delta G \frac{\bar{F}(M)}{\Delta \bar{S}}-G(M)\right)=: C .
\end{aligned}
$$

Dies ist zumindest für $i=1$ erfüllt. Es existiert also ein $\mathrm{n} \in\{1, \ldots, \mathrm{n}\}$ mit 
(5.4.7) $\quad K_{\hat{n}}<C \leqq K_{\hat{n}+1}$

und

$$
x_{i}>y_{i}, i=1, \ldots, \hat{n} .
$$

ZusammengefaBt gilt

$$
x_{i}<y_{i}, i=n+1, \ldots, N
$$

$(5.4 .8)$

$$
\begin{aligned}
& x_{i} \leqq y_{i} \quad, \quad i=\hat{n}+1, \ldots, n \\
& x_{i}>y_{i}, \quad i=1, \ldots, \hat{n} .
\end{aligned}
$$

Mit der Voraussetzung $\underline{n<N}$ folgt daraus $x L_{p} Y$.

Für den Fall $n=N$ (d.h. sowohl bei $s$ als auch bei $\hat{s}$ sind alle Gemeinden zuweisungsberechtigt) gilt für

$\underline{i}=1, \ldots, \mathrm{N}$

$$
\frac{\Delta \bar{S}_{i}}{\Delta \bar{S}}>\frac{\bar{F}_{i}(M)}{\bar{F}(M)}
$$

$(5 \cdot 4 \cdot 10)$

$$
-\frac{a \Delta G}{N a \Delta G}>\frac{a G(M)+(1-a) \bar{K}_{i}}{N a G(M)+(1-a) \sum_{i=1}^{N} \bar{K}_{i}}-\frac{\bar{K}_{i}}{N}<\frac{1}{N_{i=1}} \sum_{i}^{N} \bar{K}_{i}
$$

Mit $\overline{\mathrm{K}}_{1}<\frac{1}{\mathrm{~N}_{\mathrm{i}=1}} \sum_{\mathrm{i}}^{\mathrm{N}} \overline{\mathrm{K}}_{\mathrm{N}}<\overline{\mathrm{K}}_{\mathrm{N}}$ für $\mathrm{N}>1$ und $\overline{\mathrm{K}}_{1}<\overline{\mathrm{K}}_{\mathrm{N}}$

folgt

$$
\begin{aligned}
x_{1} & >y_{1}, \quad i=1, \ldots, \tilde{n} \\
(5.4 .11) \quad x_{i} \leqq y_{i}, & i=\tilde{n}+1, \ldots, N-1 \\
x_{i}<y_{i}, & i=N
\end{aligned}
$$


für eln $\tilde{\mathrm{n}} \in\{1, \ldots, \mathrm{N}-1\}$ mit

$$
\overline{\mathrm{K}}_{\tilde{n}}<\frac{1}{\mathrm{~N}} \sum_{i=1}^{N} \overline{\mathrm{K}}_{i} \leqq \overline{\mathrm{K}}_{\tilde{n}+1} .
$$

Daraus folgt $\mathrm{xL}_{\mathrm{p}} \mathrm{Y}$.

ם

Durch eine Erhöhung der Schlüsselmasse kann fiskalische Ungleichheit also verringert werden. Entsprechend erhöht sich die fiskalische Ungleichheit, wenn die Schlüsselmasse sinkt. Daher lassen sich im System der Schlüsselzuweisungen Verteilungsziele nicht unabhängig von der Schlüsselmasse verfolgen. Dies ist ein ziemlich ernüchterndes Resultat, denn die Schlüsselmasse ist als Instrumentvariable zur Regulierung fiskalischer Ungleichheit überhaupt nicht geeignet. Sie kann, wie wir noch sehen werden, aufgrund ihrer Beschränkung von der Einnahmenseite her nicht in dem Maße erhöht werden, wie es für fiskalische Gleichheit erforderlich wäre (s.6.1). Man kann noch nicht einmal verhindern, daß die fiskalische Ungleichheit (ungewollt) zunimmt, wenn etwa in einer Rezession die Steuereinnahmen und damit auch die Schlüsselmasse sinken sollte. 


\section{Verteilungen bei variabler Ausschüttungsquote}

In diesem Kapitel gehen wir davon aus, daß die Ausschüttungsquote beliebig variiert werden kann. Damit lassen sich (alternative) Verteilungen

$$
s(a)=\left(s_{1}(a), \ldots, s_{N}(a)\right)
$$

erzeugen, bei denen die Schlüsselzuweisungen der Gemeinden von der Ausschüttungsquote abhängen. Wir benötigen also zunächst einen funktionalen Zusammenhang zwischen der Schlüsselzuweisung irgendeiner Gemeinde und der Ausschüttungsquote.

\subsection{Die Schlüsselzuweisungen als Funktion der Ausschüt- tungsquote}

Die Schlüsselzuweisungen hängen nicht nur direkt von der Ausschüttungsquote $\mathrm{ab}$, sondern auch indirekt, weil in den Gleichungen

$$
s_{i}(a)=\theta_{i}(G(a)) a\left(E_{i} b_{i} G(a)-K_{i}\right), i=1, \ldots, N
$$

$(6.1 .1)$

$$
\text { mit } \theta_{i}(G(a))=\left\{\begin{array}{l}
0, G(a) \leqq \bar{K}_{i} \\
1, G(a)>\bar{K}_{i}
\end{array}\right.
$$

der Grundbetrag von der Ausschüttungsquote abhängt. Falls a einen bestimmten Wert $a_{0}$ hat (und auch die GröBen $M, K_{i}$ $E_{i}, b_{i}, i=1, \ldots, N$ gegeben sind), dann existiert ein eindeutiger Grundbetrag

$(6.1 .2) G_{0}:=G\left(a_{0}\right)=\frac{\frac{1}{a_{0}} M+\sum_{i=1}^{n_{0}} k_{i}}{\sum_{i=1}^{n_{c}} E_{i} b_{i}}$. 
Dabei ist vorausgesetzt, $\mathrm{da} B$ die Gemeinden nach ihrer normierten Steuerkraft in aufsteigender Reihenfolge geordnet sind und $n_{0}:=n\left(G\left(a_{0}\right)\right)$ diejenige Gemeinde in dieser ordnung ist, die gerade noch Zuweisungen erhält, d.h. es gilt:

$(6.1 .3) \quad \mathrm{E}_{\mathrm{n}_{\mathrm{o}}} \mathrm{b}_{\mathrm{n}_{\mathrm{o}}} \mathrm{G}_{\mathrm{O}}>\mathrm{K}_{\mathrm{n}_{\mathrm{O}}} \quad$ und

(6.1.4) $\quad E_{n_{0}+1} b_{n_{0}+1} G_{0} \leqq K_{n_{0}+1}$

Variiert man nun die Ausschüttungsquote, dann kann man sich leicht vorstellen, daß sich auch $G$ mit a ändert. Wir sind also an einer Funktion

$$
\begin{aligned}
\mathrm{G}: \quad(0,1] & \rightarrow \mathbb{R}_{+} \\
\mathrm{a} & \rightarrow \mathrm{G}(\mathrm{a})
\end{aligned}
$$

interessiert, die jedem a einen eindeutig bestimmten Grundbetrag $G(a)$ zuordnet. Wir werden sehen, daB sich mit a auch der Kreis der zuweisungsberechtigten Gemeinden verändert. Er wird kleiner, wenn a steigt, und größer, wenn a sinkt.

Es ist allerdings $z u$ beachten, daß es eine Zahl $\bar{n}$ von Gemeinden gibt (wir werden sie gleich spezifizieren), die bei allen zulässigen Ausgleichssätzen zuweisungsberechtigt sind. Das kommt daher, weil man von a sinnvollerweise $0<a \leqq 1$ fordern wird. Bei $a=0$ würde eine positive Schlüsselmasse bekanntlich nicht zur Verteilung kommen, weil

$$
s_{i}(a)=0 \text { für } a=0 \text { und } i=1, \ldots, N
$$

ist und die Annahme der Existenz eines endlichen Grundbetrags sofort einen Widerspruch

$$
\sum_{i=1}^{N} S_{i}(a)=0<M
$$


erzeugt. Bei $a=1$ reicht die Schlüsselmasse für $\bar{n}$ Gemeinden aus. Der Kreis der zuweisungsberechtigten Gemeinden könnte durch Erhöhung von a zwar weiter verringert werden, dann wäre die Finanzausstattung in allen zuweisungsberechtigten Gemeinden sogar höher als der Finanzbedarf. Gemeinden benötigen aber nicht mehr Finanzmittel als ihr Bedarf $B_{i}$ angibt.

Zur Spezifikation von $G(a)$ auf dem Bereich $(0,1]$ nehmen wir zunächst an, daß beim Ausgleichssatz $a_{0}$ genau $n_{0} \geqq \bar{n}$ Gemeinden Zuweisungen erhalten. Der zugehörige Grundbetrag ist durch $(6.1 .2)$ bestimmt. Durch Einsetzen in (6.1.3) und (6.1.4) erhalten wir das zugehörige Intervall für $a_{0^{\prime}}$ in dem sich die Zahl der zuweisungsberechtigten Gemeinden nicht ändert:
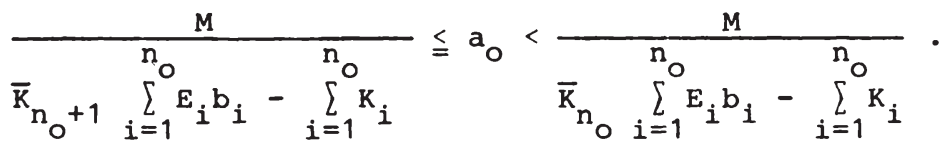

Mit (6.1.5) und $(6.1 .2)$ ist $G(a)$ auf einem Teilbereich von $(0,1]$ definiert. Betrachtet man Grundbeträge, bei denen $\mathrm{n}=\overline{\mathrm{n}}, \ldots, \mathrm{N}$ Gemeinden zuweisungen erhalten und berechnet die zugehörigen Intervalle für $a$, dann läßt sich $G(a)$ abschnittsweise definieren:

$$
G(a)=\frac{1}{a} \frac{M}{\sum_{i=1}^{n} E_{i} b_{i}}+\frac{\sum_{i=1}^{n} K_{i}}{\sum_{i=1}^{n} E_{i} b_{i}} \text { für } a \in I_{n}, n=\bar{n}, \ldots, N
$$

mit

$$
I_{n}= \begin{cases}\left(0, \bar{a}_{N}\right), & n=N \\ {\left[\underline{a}_{n}, \bar{a}_{n}\right),} & n=N-1, \ldots, \bar{n}+1 \\ {\left[\underline{a}_{\bar{n}}, 1\right],} & n=\bar{n}\end{cases}
$$


Dabei ist die Obergrenze $\bar{a}_{n}$ des Intervalls $I_{n}, n=\bar{n}+1, \ldots, N$ durch

$$
\text { (6.1.7) } \quad \bar{a}_{n}=\frac{M}{\bar{k}_{n} \sum_{i=1}^{n} E_{i} b_{i}-\sum_{i=1}^{n} k_{i}}
$$

und die Untergrenze $\underline{a}_{n}, n=\bar{n}, \ldots, N-1$ durch

$$
(6.1 .8) \quad \underline{a}_{n}=\frac{M}{\bar{k}_{n+1} \sum_{i=1}^{n} E_{i} b_{i}-\sum_{i=1}^{n} k_{i}}
$$

bestimmt.

Wir zeigen nun, $G(a)$ ist auf ganz $(0,1]$ definiert, stetig und streng monoton fallend.

\section{Definitionsbereich:}

$\mathrm{Zu}$ zeigen ist

$$
\underset{n=\bar{n}}{N} I_{n}=(0,1] \wedge \prod_{n=\bar{n}}^{N} I_{n}=\varnothing
$$

Man sieht leicht, es gilt

$$
(6.1 .9) \quad \bar{a}_{n}=a_{n-1}, n=\bar{n}+1, \ldots, N \text {, }
$$

wenn man die Identität

$$
\text { (6.1.9a) } \quad \bar{a}_{n}=\frac{M}{\bar{k}_{n} \sum_{i=1}^{n} E_{i} b_{i}-\sum_{i=1}^{n} k_{i}}=\frac{M}{\bar{k}_{n} \sum_{i=1}^{n-1} E_{i} b_{i}-\sum_{i=1}^{n-1} k_{i}}
$$


benutzt. Die Intervalle liegen also nebeneinander und decken den ganzen Bereich $(0,1)$ ab. Aus (6.1.6) und (6.1.9) folgt weiterhin, daß in einem Intervall mit größerem Ausgleichssatz die $\mathrm{Zahl}$ der zuweisungsberechtigten Gemeinden kleiner ist als in einem Intervall mit geringerem Ausgleichssatz. Wenn a steigt und Intervallgrenzen überschreitet, wird der Kreis der zuweisungsberechtigten Gemeinden kleiner.

Er umfaßt aber mindestens $\bar{n}$ Gemeinden, wobei $\bar{n}$ durch

(6.1.10) $\bar{n}=\max _{n \in\{1, \ldots, N-1\}}\left\{n \mid \bar{a}_{n+1} \leqq 1\right\}$

bestimmt ist. Aus $(6.1 .2)$ folgt

(6.1.11) $\quad \bar{a}_{\bar{n}+1} \leqq 1 \leftrightarrow M \leqq \bar{K}_{\bar{n}+1} \sum_{i=1}^{\bar{n}+1} E_{i} b_{i}-\sum_{i=1}^{\bar{n}+1} K_{i}$.

Dann erhalten Im Intervall $\left[\underline{a}_{n}, 1\right]$ genau $\bar{n}$ Gemeinden Zuweisungen, wobei wir die Obergrenze $\bar{a}_{\bar{n}}:=1$ gesetzt haben. Hätten wir

$$
\bar{a}_{\bar{n}}=\frac{M}{\bar{K}_{\bar{n}} \sum_{i=1}^{\bar{n}} E_{i} b_{i}-\sum_{i=1}^{\bar{n}} k_{i}}
$$

vereinbart, so wäre mit dem durch (6.1.10) spezifizierten $\bar{n}$ die Intervallgrenze $\bar{a}_{\bar{n}}>1$ gewesen, was bekanntlich nicht sein darf. Wie grob $\bar{n}$ aber ist, das hängt auch von $M$ ab. Denn man entnimmt $(6.1 .11)$, daß $\bar{n}$ um so größer sein muß, je größer $M$ ist. Das ist auch ganz einsichtig. Bei hohen Schlüsselmassen können selbst bei einem Ausgleichssatz von $a=1$ relativ vielen Gemeinden Schlüsselzuweisungen gewährt werden.

Nun können wir auch angeben, wie hoch die Schlüsselmasse M 
mindestens sein muB, damit bei $a=1$ alle Gemeinden Zuweisungen erhalten und somit fiskalische Gleichheit zustande kommt (siehe 4.3). Wir wissen aus (6.1.11), daß für

$$
M=\bar{K}_{N} \sum_{i=1}^{N} E_{i} b_{i}-\sum_{i=1}^{N} K_{i}
$$

genau $\bar{n}=N-1$ Gemeinden Zuweisungen erhalten und $\bar{a}_{N-1}=1$ ist. Also bekommen für alle

$(6.1 .12) \quad M>\bar{K}_{N} \sum_{i=1}^{N} E_{i} b_{i}-\sum_{i=1}^{N} K_{i}$

genau $\bar{n}=N$ Gemeinden Zuweisungen, und es ist $\underline{a}_{N}=1$.

Andererseits kann bei sehr kleinen Schlüsselmassen $\bar{n}=1$ sein, denn es gilt für alle $\bar{n} \geqq 1$

(6.1.13) $\overline{\bar{K}} \bar{n}+1 \sum_{i=1}^{\bar{n}+1} E_{i} b_{i}-\sum_{i=1}^{\bar{n}+1} K_{i}>0$.

Am linken Rand des Definitionsbereichs ist stets

$$
\bar{a}_{\mathrm{N}}>0
$$

Dies folgt zusammen mit $(6,1.9 a)$ für $\vec{n}=N-1$ sofort aus $(6.1 .13)$. Für a $\in\left(0, \bar{a}_{N}\right)$ erhalten also alle Gemeinden $z u-$ weisungen, wobei $\underline{a}_{N}:=0$ gesetzt ist.

Wir zeigen nun, $\mathrm{G}(\mathrm{a})$ ist stetig auf ganz $(0,1]$.

Klar ist, innerhalb der Intervalle $\left(\underline{a}_{n}, \bar{a}_{n}\right), n=\bar{n}, \ldots, N$ ist $G(a)$ stetig. Bleibt zu zeigen, daB $G(a)$ auch an allen (inneren) Intervallgrenzen 


$$
\bar{a}_{n}=\underline{a}_{n-1}, \bar{n}<n \leqq N
$$

stetig ist.

Der Funktionswert an den Stellen $\underline{a}_{n-1}, \bar{n}<n \leqq N$ ist

$$
G\left(\underline{a}_{n-1}\right)=\bar{K}_{n} \text {. }
$$

Dann muß mit $h>0$ und für $\bar{n}<n \leqq N$ auch

$$
\lim _{h \rightarrow 0} G\left(\bar{a}_{n}-h\right)=\bar{K}_{n}
$$

sein: Es gilt

$$
\lim _{h \rightarrow 0} G\left(\bar{a}_{n}-h\right)=\lim _{h \rightarrow 0} \frac{M\left(\frac{1}{M}\left(\bar{K}_{n} \sum_{i=1}^{n} E_{i} b_{i}-\sum_{i=1}^{n} K_{i}\right)-h\right)+\sum_{i=1}^{n} K_{i}}{\sum_{i=1}^{n} E_{i} b_{i}}=\bar{K}_{n} .
$$

Da $G(a)$ auf ganz $(0,1]$ stetig ist und $G(a)$ in allen Intervallen $\left(\underline{a}_{n}, \bar{a}_{n}\right), n=\bar{n}, \ldots, N$ wegen

$$
\frac{d G(a)}{d a}=-\frac{M}{a^{2} \sum_{i=1}^{n} E_{i} b_{i}}<0 \text { für } a \in\left(\underline{a}_{n}, \bar{a}_{n}\right)
$$

streng monoton fällt, ist $G(a)$ auch auf dem ganzen Bereich $(0,1]$ streng monoton fallend.

Wir kommen nun auf unsere ursprüngliche Fragestellung zurück und stellen unter Verwendung der Funktion $G(a)$ die Schlüsselzuwelsungen als Funktion von a dar. Dazu setzen wir $(6.1 .6)$ in $(6.1 .1)$ ein und eliminieren die Dummy-Variable $\theta_{i}(G(a))$. Wir wissen, daß für $i=\bar{n}+1, \ldots, N$ 


$$
G(a)=\bar{k}_{i}, a=\underline{a}_{i-1}=\bar{a}_{i}
$$

gilt, d.h. $\underline{a}_{i-1}$ ist ein Ausgleichssatz, bei dem die Gemeinde $i-1$ gerade noch zuweisungen erhält und die Gemeinde $i$ keine. Aufgrund der strengen Monotonie von $G(a)$ ist

$$
G(a)>\bar{k}_{i} \text { für } a<\bar{a}_{i}
$$

und

$$
G(a) \leqq \bar{K}_{i} \quad \text { furr } \quad a \geqq \bar{a}_{i}
$$

Daraus folgt für $i=\bar{n}+1, \ldots, N$

$$
\theta_{i}(a)= \begin{cases}0, & a \geqq \bar{a}_{i} \\ 1, & a<\bar{a}_{i} .\end{cases}
$$

Wir wissen, für $a=1$ erhält die Gemeinde $\bar{n}$ gerade noch $\mathrm{zu}-$ weisungen, d.h.

$$
G(1)>\bar{K}_{\bar{n}}
$$

Mit der Monotonie von $G(a)$ und mit

$$
\bar{k}_{i} \leqq \overline{\mathrm{K}}_{\overline{\mathrm{n}}} \quad, \quad i=\overline{\mathrm{n}}-1, \ldots, 1
$$

folgt

$$
\theta_{i}(a)=1, \quad 1=1, \ldots, \bar{n}
$$

Damit schreibt sich $s_{i}(a)$ ohne Dumy-Variable:

$$
\text { Für } 1=1, \ldots, \bar{n} \text { : }
$$

$$
(6.1 .14) \quad s_{i}(a)=a\left(E_{1} b_{i} G(a)-K_{i}\right) .
$$


Für $1=\bar{n}+1, \ldots, N$ :

$(6.1 .15) \quad s_{i}(a)= \begin{cases}0 & a \geqq \bar{a}_{i} \\ a\left(E_{i} b_{i} G(a)-k_{i}\right), & a<\bar{a}_{i} .\end{cases}$

Aufgrund der Stetigkeit von $G(a)$ ist $S_{i}(a)$ bei den Gemeinden $i=1, \ldots, \bar{n}$ auf ganz $(0,1]$ stetig. Da mit $h>0$

$$
\lim _{h \rightarrow 0} s_{i}\left(\bar{a}_{i}-h\right)=0=s_{i}\left(\bar{a}_{i}\right)
$$

ist, ist $s_{i}(a)$ auch bei den Gemeinden $i=\bar{n}+1, \ldots, N$ stetig auf ganz $(0,1)$.

Setzt man $G(a)$ in $(6.1 .1)$ und $(6.1 .10)$ ein, so erhält man die abschnittsweise definierten Funktionen:

Fiir $1=1, \ldots, \bar{n}:$

$$
\begin{array}{r}
\text { (6.1.16) } s_{i}(a)=\frac{M E_{i} b_{i}}{\sum_{j=1}^{n} E_{j} b_{j}}+a\left(\frac{E_{i} b_{i}}{\sum_{j=1}^{n} E_{j} b_{j}} \sum_{j=1}^{n} K_{j}-K_{i}\right), \\
a \in I_{n}, \\
n=\bar{n}, \ldots, N .
\end{array}
$$

Für $1=\bar{n}+1, \ldots, N$ :

$$
\text { (6.1.17) } s_{i}(a)=\left\{\begin{array}{l}
0, a \geqq \bar{a}_{i} \\
\frac{M E_{i} b_{i}}{\sum_{j=1}^{n} E_{j} b_{j}}+a\left(\frac{E_{i} b_{i}}{\sum_{j=1}^{n} E_{j} b_{j}} \sum_{j=1}^{n} k_{j}-k_{i}\right), \\
a \in I_{n}, \\
n=i, \ldots, N, \\
i>\bar{n} .
\end{array}\right.
$$


Halten wir fest: Es gibt eine bestimmte, genau spezifizierte Anzahl $\overline{\mathrm{n}}$ von Gemeinden, die immer Zuweisungen erhalten, das sind gleichzeitig die $\bar{n}$-ärmsten Gemeinden. Bei ihnen ist $S_{i}(a)$ abschnittsweise definiert und besteht aus lauter zusammenhängenden Geradenabschnitten. Bei den Gemeinden $i=\bar{n}+1, \ldots, N$ gibt es eine ganz bestimmte Grenze $\bar{a}_{1}$, ab der die Gemeinden keine Schlüsselzuweisungen erhalten. Diese Grenze ist für eine Gemeinde umso höher, je kleiner ihre normierte steuerkraft ist. Im Bereich $\left(0, \bar{a}_{i}\right)$ ist $s_{i}(a)$ wie bei den Gemeinden $i=1, \ldots, \bar{n}$ definiert. 


\subsection{Optimale Ausschüttungsquoten für Kommunen}

Wir interessieren uns nun für eine Verteilung

$$
s\left(a_{i}^{*}\right)=\left(s_{1}\left(a_{i}^{*}\right), \ldots, s_{i}\left(a_{i}^{*}\right), \ldots s_{N}\left(a_{i}^{*}\right)\right)
$$

mit der Ausschüttungsquote $a_{i}^{*}$, bei der eine Gemeinde $i$ ihre Finanzausstattung

$$
\text { (6.2.1) } \quad F_{i}(a):=S_{i}(a)+K_{i}
$$

maximiert. Die Quote $a_{i}^{*}$ erfüllt also die Optimalbedingung

$$
\text { (6.2.2) } \quad F_{i}\left(a_{i}^{*}\right)=\max _{a \in(0,1]} F_{i}(a) \text {. }
$$

Mit der Äquivalenz

(6.2.3) $\max _{a} F_{i}(a) \oplus \max _{a} S_{i}(a)$

maximieren die Gemeinden ihre Finanzausstattung durch Maximierung ihrer Schlüsselzuweisung, d.h.

(6.2.4) $\quad S_{i}\left(a_{i}^{*}\right)=\max _{a} S_{i}(a)$.

Ist eine Gemeinde auf ihre relative Position bedacht, dann maximiert sie ihren Finanzanteil

$(6.2 .5)$

$$
A_{i}(a):=\frac{F_{i}(a)}{\sum_{j=1}^{N} F_{j}(a)}=\frac{S_{i}(a)+K_{i}}{K+M}
$$

ebenfalls durch Maximierung ihrer Schlüsselzuweisung. Wie man in (6.2.5) sieht, gilt nämlich 
$(6.2 .6) \quad \max _{a} A_{i}(a) \rightarrow \max _{a} S_{i}(a)$

und für $a_{i}^{*}$ aus $(6.2 .2)$ somit

$$
A_{i}\left(a_{i}^{*}\right)=\max _{a} A_{i}(a)
$$

Das ist auch sehr einsichtig. Die Summe der Finanzausstattungen ist konstant. Die Maximierung der Finanzausstattung einer Gemeinde bedeutet damit automatisch auch einen maximalen Finanzanteil, denn jeder Anstieg der Finanzausstattung einer Gemeinde ist nur auf Kosten einer anderen möglich. Da die Summe der Steuerkraft $K$ ohnehin konstant ist, können sich die Gemeinden auf die Maximierung ihrer Schlüsselzuweisung beschränken, um sich absolut und relativ am günstigsten $z u$ stellen.

Untersuchen wir zunächst die Funktion $S_{i}(a)$ für die Gemeinden $i=\bar{n}+1, \ldots, N$.

Klar ist, das Maximum kann nicht im Bereich $\left[\bar{a}_{i}, 1\right]$ liegen, da dort die Gemeinden keine positiven Zuweisungen erhalten. Im Bereich $\left(0, \bar{a}_{i}\right)$ besteht $s_{i}(a)$ aus lauter Geradenabschnitten und ist überall stetig.

In den offenen Intervallen $\left(\underline{a}_{n}, \bar{a}_{n}\right), n=i, \ldots, N$ ist die Steigung

$$
\frac{d S_{1}(a)}{d a}=E_{i} b_{i} \frac{\sum_{j=1}^{n} K_{j}}{\sum_{j=1}^{n} E_{j} b_{j}}-K_{i}
$$

konstant und daher entweder positiv oder negativ oder horizontal (denn einer der Fälle muß ja eintreten). 
Im Intervall $\left(\underline{a}_{i}, \bar{a}_{i}\right), i=\bar{n}+1, \ldots, N$ ist sie nicht positiv:

(6.2.8) $\frac{d S_{i}(a)}{d a}=E_{i} b_{i} \frac{\sum_{j=1}^{i} K_{j}}{\sum_{j=1}^{i} E_{j} b_{j}}-k_{i} \leqq 0-\frac{\sum_{j=1}^{i} K_{j}}{\sum_{j=1}^{i} E_{j} b_{j}} \leqq \bar{k}_{i}$.

Beweis für $i \geqq 1$ durch vollständige Induktion:

$i=1: \overline{\mathrm{K}}_{1} \leqq \overline{\mathrm{K}}_{1}$

$i=2: \frac{k_{1}+K_{2}}{E_{1} b_{1}+E_{2} b_{2}} \leqq \frac{k_{2}}{E_{2} b_{2}} \leftrightarrow \bar{k}_{1} \leqq \bar{K}_{2}$

$i=n: \quad \sum_{j=1}^{n} k_{j} / \sum_{j=1}^{n} E_{j} b_{j} \leqq \bar{k}_{n}$

$i=n+1: \quad \sum_{j=1}^{n+1} k_{j} / \sum_{j=1}^{n+1} E_{j} b_{j} \leqq \bar{k}_{n+1} \bullet \sum_{j=1}^{n} K_{j} / \sum_{j=1}^{n} E_{j} b_{j} \leqq \bar{k}_{n+1}$.

Diese Aquivalenz folgt unter Benutzung der Annahme aus:

$$
\sum_{j=1}^{n} K_{j} / \sum_{j=1}^{n} E_{j} b_{j} \leqq \bar{k}_{n} \leqq \bar{k}_{n+1}
$$

口

Wenn a sinkt und sukzessive die Intervalle $\left(\underline{a}_{n}, \bar{a}_{n}\right)$, $\mathrm{n}=i+1, \ldots, \mathrm{N}$ durchläuft, dann wird die Steigung beim Uberschreiten einer Intervallgrenze nicht kleiner. Mit 


$$
\begin{aligned}
& \frac{d S_{i}(a)}{d a}=\frac{E_{i} b_{i}}{\sum_{j=1}^{n} E_{j} b_{j}} \sum_{j=1}^{n} K_{j}-K_{i}, a \in\left(\underline{a}_{n}, \bar{a}_{n}\right), n=i+1, \ldots, N \\
& \frac{d S_{i}(a)}{d a}=\frac{E_{i} b_{i}}{\sum_{j=1}^{n+1} E_{j} b_{j}} \sum_{j=1}^{n+1} K_{j}-K_{i}, a \in\left(\underline{a}_{n+1}, \bar{a}_{n+1}\right), n=i+1, \ldots, N-1
\end{aligned}
$$

muß also

$(6.2 .9)$

$$
\frac{E_{i} b_{i}}{\sum_{j=1}^{n} E_{j} b_{j}} \sum_{j=1}^{n} K_{j}-K_{i} \leqq \frac{E_{i} b_{1}}{\sum_{j=1}^{n+1} E_{j} b_{j}} \sum_{j=1}^{n+1} K_{j}-K_{i}
$$

gelten. Die Bedingung $(6.2 .9)$ ist äquivalent mit dem Ausdruck

$$
\frac{\sum_{j=1}^{n} K_{j}}{\sum_{j=1}^{n} E_{j} b_{j}} \leqq \bar{k}_{n+1}
$$

der, wie der Beweis von (6.2.8) gezeigt hat, für alle $n \geqq 1$ erfüllt ist.

Da mit (6.2.8) und (6.2.9) die Steigung im Intervall $\left(\underline{a}_{i}, \bar{a}_{i}\right)$ nicht positiv ist und in Intervallen mit geringerem a nicht kleiner wird (höchstens gröBer), ist das optimale a genau die Grenze der Intervalle, an der die negative steigung in eine positive übergeht:

Falls also ein $n^{*} \in\{i+1, \ldots, N-1\}$ existiert mit 


$$
\frac{d S_{i}(a)}{d a}<0, a \in\left(\underline{a}_{n} *, \bar{a}_{n} *\right)
$$

$(6.2 .10)$ und

$$
\frac{d S_{i}(a)}{d a}>0, a \in\left(\underline{a}_{n}{ }^{*}+1, \bar{a}_{n}{ }^{*}+1\right) \text {, }
$$

dann ist

$$
\text { (6.2.11) } \quad a_{i}^{*}=\underline{a}_{n} *=\bar{a}_{n} *+1 \text {. }
$$

Die Bedingung $(6.2 .10)$ ist äquivalent mit

$$
\text { (6.2.12) } \frac{\sum_{j=1}^{n^{*}} K_{j}}{\sum_{j=1}^{n^{*}} E_{j} b_{j}}<\bar{K}_{i}<\frac{\sum_{j=1}^{n^{*}+1} K_{j}}{\sum_{j=1}^{*+1} E_{j} b_{j}}
$$

Falls ein $n * \in\{i, \ldots, N\}$ existiert mit

$$
(6.2 .13) \quad \frac{\mathrm{dS}_{i}(a)}{d a}=0, a \in\left(\underline{a}_{n *}, \bar{a}_{n^{*}}\right) \text {, }
$$

dann ist mit $\mathbb{A}_{i}^{*}:=\left\{a_{i}^{*}\right\}$

$(6.2 .14) \mathbb{A}_{1}^{*}=\left\{\begin{array}{cl}{\left[\underline{a}_{n *}, \bar{a}_{n *}\right],} & n * \in\{i, \ldots, N-1\} \\ \left(0, \bar{a}_{N}\right], & n *=N\end{array}\right.$ 
Die Bedingung $(6.2 .13)$ ist äquivalent mit

(6.2.15) $\quad \bar{k}_{i}=\frac{\sum_{j=1}^{n^{*}} K_{j}}{\sum_{j=1}^{n^{*}} E_{j} b_{j}}$.

Sie wird nur zufällig erfüllt sein, da die rechte Seite von (6.2.15) nur diskrete Werte annehmen kann.

Falls im ersten Intervall

$(6.2 .16) \quad \frac{d_{i}(a)}{d a}<0, \quad a \in\left(0, \bar{a}_{N}\right)$,

d.h. $\quad \bar{k}_{i}>\frac{\sum_{j=1}^{N} k_{j}}{\sum_{j=1}^{N} E_{j} b_{j}}$

gilt, dann existiert ein $n^{*}$ mit den Eigenschaften (6.2.10) bzw. (6.2.13) nicht, weil $s_{i}$ auf $\left(0, \bar{a}_{i}\right)$ streng monoton fällt. Das Optimum ist also am linken Rand des Definitionsbereichs. Grob gesprochen könnte man sagen, der optimale Ausgleichsatz ist ein sehr kleiner wert nahe bei Null. Da der Definitionsbereich aber linksoffen ist, existiert $a_{i}^{*}$ im streng mathematischen Sinne nicht. Der Definitionsbereich kann auch nicht stetig un $a=0$ erweitert werden. Sinnvollerweise wäre $S_{i}(0):=0 \mathrm{zu}$ setzen, was sofort $z u$ einer Unstetigkeitsstelle bei $a=0$ führt. Man kann aber die maximale Zuweisung als Grenzwert angeben:

(6.2.17) $s_{i}^{*}=\lim _{\substack{h \rightarrow 0 \\ h>0}} s_{i}(0+h)=\frac{M_{i} b_{i}}{\sum_{j=1}^{N} E_{j} b_{j}}$. 
Die Bedingung $(6.2 .16)$ ist zumindest für die Gemeinde $i=\mathrm{N}$ erfüllt und kann durchaus auch noch von weiteren reichen Gemeinden erfüllt werden.

Wir bestimmen das Optimum nun für die Gemeinden $i=1, \ldots, \bar{n}$. Bei ihnen besteht $S_{i}(a)$ aus lauter zusammenhängenden Geradenabschnitten, deren steigung wegen (6.2.9) nicht kleiner wird, wenn a sinkt (und dabei Intervallgrenzen überschreitet).

Falls also im letzten Intervall

(6.2.18) $\frac{d S_{i}(a)}{d a}>0, a \in\left[\underline{a}_{n}, 1\right]$

gilt, dann ist

(6.2.19) $\quad a_{i}^{*}=1$

und

$$
s_{i}\left(a_{i}^{*}\right)=E_{i} b_{i} \frac{M+\sum_{j=1}^{\bar{n}} k_{j}}{\sum_{j=1}^{\bar{n}} E_{j} b_{j}}-k_{i} .
$$

Die Bedingung $(6.2 .18)$ ist äquivalent mit

$(6.2 .20) \quad \bar{k}_{i}<\frac{\sum_{j=1}^{\bar{n}} K_{j}}{\sum_{j=1}^{n} E_{j} b_{j}}$.

Für die Gemeinde $i=\bar{n}$ ist die Bedingung (6.2.20) nicht erfüllt, weil wir in (6.2.8) genau das Gegenteil bewiesen haben. Sie wird aber von $i=1$ erfüllt (für $\bar{n}>1$ und 
$\overline{\mathrm{K}}_{1}<\overline{\mathrm{K}}_{2}$; Beweis durch vollständige Induktion nach $\overline{\mathrm{n}}$ ). Es existiert also ein $\hat{n} \in\{1, \ldots, \bar{n}-1\}$ mit

$(6.2 .21) \quad \bar{K}_{\hat{n}}<\frac{\sum_{j=1}^{\bar{n}} k_{j}}{\sum_{j=1}^{n} E_{j} b_{j}} \leqq K_{\hat{n}+1}$

und

(6.2.22) $a_{i}^{*}=1, i=1, \ldots, \hat{n}$

Für die Gemeinden $i=\hat{n}+1, \ldots, \bar{n}$ führen die gleichen Uberlegungen wie bei den Gemeinden $i=\bar{n}+1, \ldots, N$ zu Kriterien, die analog $z u(6.2 .12)$ und (6.2.15) gelten, nur ist das Maximum diesmal auf dem ganzen Bereich $(0,1] \mathrm{zu}$ suchen. Es gelten die Kriterien:

(6.2.23) Falls ein $n^{*} \in\{\bar{n}, \ldots, N-1\}^{1)}$ existiert, das (6.2.12) erfüllt, dann ist $a_{i}^{*}$ durch (6.2.11) bestimmt.

(6.2.24) Falls (6.2.15) für $n * \in\{\bar{n}, \ldots, N-1\}$ erfüllt ist, dann folgt

$$
\mathbb{A}_{i}^{*}= \begin{cases}{\left[\underline{a}_{\bar{n}}, 1\right],} & n *=\bar{n} \\ {\left[\underline{a}_{n}, \bar{a}_{n}\right],} & n * \in\{\bar{n}+1, \ldots, N-1\} \\ \left(0, \bar{a}_{N}\right], & n *=N .\end{cases}
$$

Im Gegensatz $z u$ den Gemeinden $i=\bar{n}+1, \ldots, N$ gibt es immer ein $n^{*}$, das $(6.2 .12)$ oder $(6.2 .15)$ erfüllt, wenn man an-

1) Man beachte den Unterschied: Bei $i=\bar{n}+1, \ldots, N$ wurde $n * \epsilon\{i+1, \ldots, N-1\}$ mit $i>\bar{n}$ gefordert. 
nimmt, daß für ein $\tilde{n} \geqq \bar{n}$

$$
\bar{K}_{\tilde{n}} \leqq \frac{\sum_{j=1}^{N} K_{j}}{\sum_{j=1}^{N} E_{j} b_{j}}
$$

gilt, also (6.2.16) nicht erfüllt werden kann.

Abschließend wollen wir die optimalen Ausschüttungsquoten der Gemeinden miteinander vergleichen. Dazu nehmen wir noch vereinfachend an, daß die optimale Ausschüttungsquote bei jeder Gemeinde eindeutig sei. Für Gemeinden, die (6.2.15) bzw. (6.2.24) erfüllen, gelte

(6.2.25) $\quad a_{i}^{*}=\left\{\begin{array}{l}\underline{a}_{n *}, n^{*} \in\{\bar{n}, \ldots, N-1\} \\ \bar{a}_{N}, n^{*}=N .\end{array}\right.$

Die verschiedenen Kriterien lassen sich zusammenfassen, wenn man die Intervalle

$$
L_{n}=\left[\underline{L}_{n}, \bar{L}_{n}\right), n=\bar{n}, \ldots, N-1
$$

mit

$$
\underline{L}_{n}:=\frac{\sum_{j=1}^{n} K_{j}}{\sum_{j=1}^{n} E_{j} b_{j}} \quad \text { und } \quad \bar{L}_{n}:=\frac{\sum_{j=1}^{n+1} K_{j}}{\sum_{j=1}^{n+1} E_{j} b_{j}}
$$

definiert und $n^{*}:=n$ setzt.

Dann gilt für die optimalen Ausschüttungsquoten der Gemeinden $i=1, \ldots, N$ : 


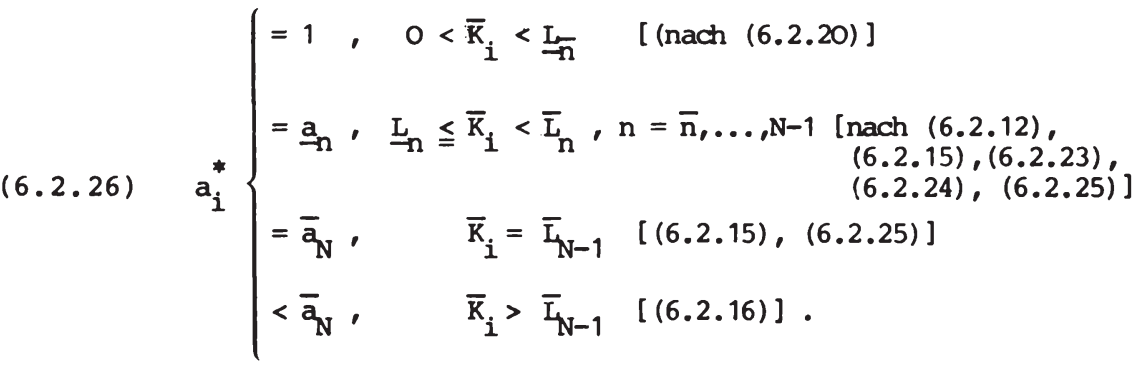

Man kann sehr schön erkennen, daß die optimale Ausschüttungsquote einer Gemeinde $i$ nicht kleiner ist als die einer Gemeinde $j$, wenn $i$ nicht reicher als $j$ ist, es gilt also

$(6.2 .27) \quad \overline{\mathrm{K}}_{1} \leqq \overline{\mathrm{K}}_{2} \leqq \cdots \leqq \overline{\mathrm{K}}_{\mathrm{N}} \Rightarrow \mathrm{a}_{1}^{*} \geqq \mathrm{a}_{2}^{*} \geqq \cdots \geqq \mathrm{a}_{\mathrm{N}}^{*} \cdot$

6.3 Die Variation der Ausschüttungsquote aus der Sicht von Gemeindegruppen

In diesem Abschnitt gehen wir von einem beliebigen, aber fixen Ausgleichsatz aus und fragen, wie sich die Finanzausstattung und der Finanzanteil von Gemeinden und Gemeindegruppen verändert, wenn sich der Ausgleichsatz um einen bestimmten Wert $\Delta a$ verändert.

\subsubsection{Die Umverteilung der Finanzausstattungen und Finanz- anteile}

Nehmen wir einen Ausgleichsatz a $\in I_{n}$ an mit $n \in\{\bar{n}, \ldots, N\}$ beliebig und

$$
I_{n}= \begin{cases}\left(0, \bar{a}_{N}\right), & n=N \\ {\left[\underline{a}_{n}, \bar{a}_{n}\right),} & n=N-1, \ldots, \bar{n}+1 \\ {\left[\underline{a}_{\bar{n}}, 1\right],} & n=\bar{n},\end{cases}
$$


wobei $\underline{a}_{n}, \bar{a}_{n}$ durch $(6.1 .8)$ bzw. (6.1.7) und $\bar{n}$ durch $(6.1 .10)$ bestimmt sind. Dann ist bekanntlich

$(6.3 \cdot 1)$

$$
G(a)=\frac{\frac{M}{a}+\sum_{i=1}^{n} K_{i}}{\sum_{i=1}^{n} E_{i} b_{i}}
$$

der zugehörige Grundbetrag, und $n$ ist die Zahl der zuweisungsberechtigten Gemeinden (siehe (6.1.6)).

Für a könnte man etwa den zur zeit gesetzlich vorgeschriebenen Ausgleichssatz nehmen. Dann ließe sich sehr schön die Veränderung des status quo, also der derzeitigen Finanzausstattung bzw. des derzeitigen Finanzanteils der Gemeinden analysieren.

Wir wollen an dieser stelle noch nicht konkret festlegen, wie groß $\Delta a$ genau ist, wir müssen nur die Restriktion

$(6.3 .2)$

$$
(a+\Delta a) \in(0,1] \Rightarrow \Delta a \in(-a, 1-a]
$$

beachten. Da a ein Prozentsatz ist und $\Delta a$ deshalb als Prozentpunkte interpretiert werden muB, ist es für die weitere Analyse manchmal etwas geschickter,

(6.3.3) $\lambda \mathrm{a}:=\mathrm{a}+\Delta \mathrm{a} \Rightarrow \lambda:=1+\frac{\Delta \mathrm{a}}{\mathrm{a}}$

zu setzen mit der Restriktion

$(6.3 .4) \quad \lambda \in\left(0, \frac{1}{a}\right]$.

Dann läßt sich der neue Ausgleichssatz als das $\lambda$-fache des alten Ausgleichssatzes interpretieren, und es gilt: 
$(6.3 .5)$

$$
\lambda<1 \leftrightarrow \Delta \mathrm{a}<0
$$

$\lambda<1, \Delta a<0$

$$
\lambda>1 \leftrightarrow \Delta a>0 .
$$

Die Änderung der Schlüsselzuweisung einer Gemeinde $i$ in Abhängigkeit von $\lambda$ ist durch

$$
\text { (6.3.6) } \quad \Delta S_{i}(\lambda):=S_{i}(\lambda a)-S_{i}(a),
$$

und die Ainderung der Finanzausstattung einer Gemeinde i bei $\lambda$ durch

$$
\text { (6.3.7) } \quad \Delta F_{i}(\lambda):=F_{i}(\lambda a)-F_{i}(a)
$$

definiert. Mit der Definitionsgleichung (6.2.1) sieht man sofort:

(6.3.8) $\Delta F_{i}(\lambda)=\Delta S_{i}(\lambda)$, für $i=1, \ldots, N, \lambda \in\left(0, \frac{1}{a}\right]$.

Vergleicht man die Finanzanteile einer Gemeinde vor und nach der Anderung der Ausschüttungsquote, so erhält man mit (6.2.5)

$(6.3 .9)$

$$
A_{i}(\lambda a)>A_{i}(a) \leftrightarrow \Delta S_{i}(\lambda)>0
$$

$$
A_{i}(\lambda a) \leqq A_{i}(a) \quad-\Delta S_{i}(\lambda) \leqq 0
$$

Um die Veränderung der Finanzausstattung oder des Finanzanteils der Gemeinden zu analysieren, genügt es also, die veränderung der Schlüsselzuweisungen zu betrachten. Zur Berechnung der $\Delta \mathrm{S}_{\underline{i}}(\lambda)$ unterscheiden wir wieder die Gemeindegruppen $\left\{1, \ldots, \frac{1}{n}\right\}$ und $\{\bar{n}+1, \ldots, N\}$.

Erinnern wir uns, bei den Gemeinden $i=1, \ldots, \bar{n}$ gilt allgemein für a $\in(0,1]$ 
$(6.1 .14)$

$$
S_{i}(a)=a\left(E_{i} b_{i} G(a)-K_{i}\right)
$$

Man erhält für alle $\lambda$

(6.3.10) $\Delta S_{i}(\lambda)=a E_{i} b_{i}(\lambda G(\lambda a)-G(a))+a(1-\lambda) K_{i}$,

wobei der Wert $G(a)$ durch (6.3.1) und die Funktion $G(\lambda a)$ durch (6.1.6) spezifiziert sind. (Die Interpretation von (6.3.10) ist etwas kompliziert und erfordert den Beweis zusätzlicher Lemmata. Wir müssen sie daher noch etwas zurückstellen.)

Bei den Gemeinden $i=\bar{n}+1, \ldots, N$ gilt allgemein für a $\in(0,1]$ :

(6.1.15) $\quad s_{i}(a)= \begin{cases}0 & , a \geqq \bar{a}_{i} \\ a\left(E_{i} b_{i} G(a)-K_{i}\right), a<\bar{a}_{i} .\end{cases}$

Wir müssen daher unterscheiden, ob $a<\bar{a}_{i}$ (Fall a) oder $a \geqq \bar{a}_{i}($ Fall b) ist.

Fall a): $\quad a<\bar{a}_{1}$

Es ergibt sich

(6.3.11) $\Delta S_{i}(\lambda)= \begin{cases}a E_{i} b_{i}(\lambda G(\lambda a)-G(a))+a(1-\lambda) K_{i}, & \lambda<\frac{\bar{a}_{i}}{a} \\ -a\left(E_{i} b_{i} G(a)-k_{i}\right) & , \lambda \geqq \frac{\bar{a}_{i}}{a}>1 .\end{cases}$

Die Interpretation ist nur für den zweiten Ast einfach: Falls sich der Ausgleichssatz mindestens um ein bestimmtes Vielfaches (nämlich das $\left.\bar{a}_{i} / a-f a c h e\right)$ erhöht, dann scheidet die Gemeinde $i$ aus dem Kreis zuweisungsberechtigter Gemeinden aus. Die Differenz $\Delta S_{i}(\lambda)$ ist negativ und entspricht 
betragsmäßig genau den Schlüsselzuweisungen, die die Gemeinde vorher erhalten hatte. Falls der neue Satz nicht so stark gestiegen ist oder kleiner ist als der alte, dann erhält die Gemeinde $i$ auch beimneuen Satz zuweisungen. Die Differenz hat die gleiche Form wie bei den Gemeinden $i=1, \ldots, \bar{n}$. Wir werden darauf später ausführlich eingehen. $\underline{\text { Fall b) }}: \quad a \geqq \bar{a}_{i} \quad-\frac{\bar{a}_{i}}{a} \leqq 1, s_{i}(a)=0$ liefert (6.3.12) $\Delta S_{i}(\lambda)= \begin{cases}0 & , \lambda \geqq \frac{\bar{a}_{i}}{a} \\ \lambda a\left(E_{i} b_{i} G(\lambda a)-k_{i}\right), & \lambda<\frac{\bar{a}_{1}}{a} .\end{cases}$

Wenn a um einen genügend hohen Faktor (d.h. um mehr als das $\bar{a}_{i}$ /a-fache) vermindert wird, dann kommt die Gemeinde $i$ zum Kreis der zuweisungsberechtigten Gemeinden hinzu und $\Delta S_{i}(\lambda)$ entspricht der Schlüsselzuweisung, die sie beim neuen Ausgleichssatz $\lambda$ a erhält. Steigt a oder fällt er nicht so stark, dann erhält $i$ auch beim neuen Satz keine Zuweisungen und $\Delta S_{i}(\lambda)=0$.

Für eine explizite Darstellung der Funktionen $\Delta S_{i}(\lambda)$ müBte man nun in den fällen a) und b) $G(a)$ bzw. $G(\lambda a)$ durch den Funktionswert bzw. durch die Funktionsgleichung ersetzen. Dies ist aber sehr aufwendig und wird für unsere weitere Analyse auch nicht benötigt. Wir wollen deshalb nicht näher darauf eingehen, sondern gleich die Auswirkungen einer Erhöhung oder Verringerung der Ausschüttungsquote auf verschiedene Gemeinden und Gemeindegruppen miteinander vergleichen. 
Dazu gehen wir im folgenden zusätzlich zur Annahme eines fixen Ausgleichssatzes a auch von einer ganz bestimmten Erhöhung bzw. Verringerung von a um den (fixen) Faktor $\lambda$ aus. Eine solche Veränderung des status quo wird sich bei verschiedenen Gemeinden natürlich ganz verschieden auswirken, einfach deshalb, weil die Grenze $\bar{a}_{i}, a b$ der eine Gemeinde keine zuweisungen mehr erhält, in Gemeinden mit unterschiedlicher normierter steuerkraft ganz unterschiedlich ist. Damit befinden sich die Gemeinden auch in unterschiedlichen Situationen, je nachdem, ob der neue Ausgleichssatz im Vergleich zum alten bei einer Erhöhung (Verringerung) die Grenze $\overline{\mathrm{a}}_{i}$ überschreitet (unterschreitet), oder ob beide Sätze darunter bzw. darüber bleiben. Die Gemeinden sind danach so in Gruppen einzuteilen, daB einer Gruppe diejenigen Gemeinden angehören, die sich in der gleichen Situation befinden. Betrachten wir zunächst eine Verringerung der Ausschüttungsquote.

\subsubsection{Verringerung der Ausschüttungsquote}

Sei $\lambda \in J_{n+k}, n \in\{1, \ldots, N\}$ und $\lambda<1$ fest mit

(6.3.13) $J_{n+k}= \begin{cases}{\left[\frac{\underline{a}_{n+k}}{a}, \frac{\bar{a}_{n+k}}{a}\right),} & 0 \leqq k \leqq N-n-1 \\ \left(0, \frac{\bar{a}_{N}}{a}\right) & , k=N-n .\end{cases}$

Dann gibt $\mathrm{k}$ wieder die Zahl der Gemeinden an, die zum Kreis der zuweisungsberechtigten Gemeinden neu hinzukommen, denn aufgrund der strengen Monotonie von $G(a)$ ist

$$
\text { (6.3.14) } \lambda<1 \quad k \geqq 0 \text {. }
$$

Der zugehörige Grundbetrag ist mit $(6.1 .6)$ durch 
$(6.3 .15) \quad G(\lambda a)=\frac{\frac{M}{\lambda a}+\sum_{i=1}^{n+k} K_{i}}{\sum_{i=1}^{n+k} E_{i} b_{i}}$

bestimmt.

Wir können nun drei Gruppen von Gemeinden unterscheiden:

\section{A Gemeinden $i=1, \ldots, n$ :}

Sie erhalten bei a und $\lambda a$ Zuweisungen, weil mit $\bar{a}_{i}=\left\{a \mid G(a)=\bar{k}_{i}\right\}$

(6.3.16) $\lambda a<a<\bar{a}_{i}$

gilt. $\mathrm{Zu}$ dieser Gruppe gehören natürlich auch die Gemeinden $i=1, \ldots, \bar{n}$ mit $\bar{n} \leqq n$, die ohnehin bei jedem a $\in(0,1] z u-$ weisungsberechtigt sind, weil bekanntlich stets

$$
G(a)>\bar{K}_{i} \quad \text { flir } i=1, \ldots, \bar{n} \quad \text { und } a \leqq 1
$$

erfüllt ist.

Aus (6.3.11) entnimmt man

$$
\text { (6.3.17) } \Delta S_{i}=a E_{i} b_{i}(\lambda G(\lambda a)-G(a))+a(1-\lambda) K_{i} \text {. }
$$

B Gemeinden $i=n+1, \ldots, n+k$ :

Diese Gemeinden haben vorher keine Zuweisungen erhalten, sind aber nachher zuweisungsberechtigt, weil

$$
(6.3 .18) \quad \lambda a<\bar{a}_{i} \leqq a
$$


gilt.

Aus (6.3.12) entnimmt man
(6.3.19) $\Delta S_{i}=\lambda a\left(E_{i} b_{i} G(\lambda a)-K_{i}\right) \cdot$
C Gemeinden $i=n+k+1, \ldots, N$ :

Für sie gilt

$(6.3 .20) \quad \bar{a}_{i} \leqq \lambda a<a ，$

d.h. sie erhalten auch nach der Verringerung des Ausgleichssatzes noch keine zuweisungen und (6.3.12) liefert

(6.3.21) $\quad \Delta \mathrm{S}_{i}=0$.

Zusammenfassend kann man schreiben:

(6.3.22) $\Delta S_{i}= \begin{cases}0 & , i=n+k+1, \ldots, N \\ \lambda a\left(E_{i} b_{i} G(\lambda a)-k_{i}\right), & i=n+1, \ldots, n+k \\ a E_{i} b_{i}(\lambda G(\lambda a)-G(a))+a(1-\lambda) k_{i}, & i=1, \ldots, n .\end{cases}$

Die Interpretation ist nun für die Gruppen $B$ und $C$ recht einfach. Bei den Gemeinden $\{n+k+1, \ldots, N\}$, das sind bekanntlich die reichen Gemeinden, verändern sich die Finanzausstattung und auch der Finanzanteil nicht. Beide nehmen aber zu bei den Gemeinden $\{n+1, \ldots, n+k\}$.

Bei der Gruppe A der armen Gemeinden gilt

(6.2.23) $\Delta S_{i}<0 \Leftrightarrow \bar{K}_{i}<\frac{1}{1-\lambda}(G(a)-\lambda G(\lambda a))$.

Folgende einfache Uberlegung zeigt, daß es mindestens eine 
Gemeinde gibt, deren Finanzausstattung und Finanzanteil sinken, wobei wir noch $\mathrm{k}>0$ voraussetzen. ${ }^{1)}$

Wie wir wissen, gilt:

$$
\sum_{i=1}^{N} s_{i}(a)=\sum_{i=1}^{N} s_{i}(\lambda a)=M
$$

Daraus folgt

$$
\sum_{i=1}^{N} \Delta s_{i}=0
$$

und mit $\sum_{i=n+k+1}^{N} \Delta S_{i}=0$ folgt weiterhin

$$
\sum_{i=1}^{n} \Delta S_{i}=-\sum_{i=n+1}^{n+k} \Delta S_{i}
$$

Da $\Delta S_{i}>0$ ist für alle $i=n+1, \ldots, n+k$, muß $\Delta S_{i}<0$ sein für mindestens ein $i \in\{1, \ldots, n\}$.

Das ist auch sehr einsichtig. Aufgrund der konstanten Schlüsselmasse geht jede Erhöhung der Schlüsselzuweisung einer Gemeinde nur auf Kosten mindestens einer anderen Gemeinde. Da aber alle Gemeinden der Gruppe $\{n+1, \ldots, n+k\}$ von einer Verringerung des Ausgleichssatzes profitieren, mu mindestens eine Gemeinde aus der Gruppe $\{1, \ldots, n\}$ schlechter gestellt werden.

1) Diese Annahme ist nicht sehr restriktiv. Sie besagt lediglich, daß a und $\lambda a$ nicht im gleichen Intervall liegen, d.h. der Ausgleichssatz so weit gekürzt ist, daß mindestens eine Gemeinde zum Kreis der zuweisungsberechtigten Gemeinden hinzukommt. Der Fall k = 0 wird anschließend separat behandelt. 
Es gibt also ein $\hat{n} \in\{1, \ldots, n\}$ mit

(6.3.24) $\quad \overline{\mathrm{K}}_{\hat{\mathrm{n}}}<\frac{1}{1-\lambda}(\mathrm{G}(\mathrm{a})-\lambda \mathrm{G}(\lambda \mathrm{a})) \leqq \overline{\mathrm{K}}_{\hat{\mathrm{n}}+1}$

und

(6.3.25) $\Delta S_{i}<0$ für alle $i=1, \ldots, \hat{n}$.

Bemerkenswert ist, es sind die ärmsten der armen Gemeinden, die bei der Umverteilung der Schlüsselzuweisungen benachteiligt werden. Denn es kann unter den Gemeinden $\{1, \ldots, n\}$ durchaus auch welche geben, die besser gestellt werden. Das sind die Gemeinden, für die

$(6.3 .26) \quad \bar{K}_{i} \geqq \frac{1}{1-\lambda}(G(a)-\lambda G(\lambda a))$

erfüllt ist, also die Gemeinden $\{\hat{n}+1, \ldots, n\}$. Für sie ist

$$
\Delta s_{i} \geqq 0
$$

Notwendige ${ }^{1)}$ Voraussetzung dafür, daß es solche Gemeinden aber überhaupt gibt, ist die Bedingung

$$
\frac{1}{1-\lambda}(G(a)-\lambda G(\lambda a))<G(a) \rightarrow G(a)<G(\lambda a),
$$

die aufgrund der strengen Monotonie von $G(a)$ beim vorliegenden Fall $\lambda<1$ aber immer erfüllt ist.

Das Gleichheitszeichen in (6.3.26) gilt nur zufällig, da

1) Diese Bedingung ist aber nicht hinreichend, weil

$$
\overline{\mathrm{K}}_{\mathrm{n}}<\frac{1}{1-\lambda}(\mathrm{G}(\mathrm{a})-\lambda \mathrm{G}(\lambda \mathrm{a}))<\mathrm{G}(\mathrm{a}) \text { sein kann. }
$$


auf der rechten Seite der Ungleichung ein diskreter Wert steht und es eine Gemeinde mit identischer normierter steuerkraft nicht geben muB.

Es gilt aber sicher

$$
\Delta s_{i}>0 \text { für } \hat{n}+2, \ldots, n \text {, }
$$

falls

$$
\overline{\mathrm{K}}_{\hat{\mathrm{n}}+1}<\overline{\mathrm{K}}_{\hat{\mathrm{n}}+2} \text { gilt. }
$$

Der Einfachheit halber nehmen wir im folgenden an, daß auch $\Delta S_{i}>0$ ist für $i=\hat{n}+1$. Damit ergibt sich insgesamt

$(6.3 .27) \quad \Delta F_{i}=\Delta s_{i} \begin{cases}<0, & i=1, \ldots, \hat{n} \\ >0 & , i=\hat{n}+1, \ldots, n+k \\ =0, & i=n+k+1, \ldots, N\end{cases}$

und

(6.3.28) $\quad A_{i}(\lambda a) \quad\left\{\begin{array}{l}<A_{i}(a), i=1, \ldots, \hat{n} \\ >A_{i}(a), i=\hat{n}+1, \ldots, n+k \\ =A_{i}(a), i=n+k+1, \ldots, N\end{array}\right.$

Betrachten wir nun noch den (unbedeutenden) Spezialfall $\underline{k}=0, d . h$. die Verringerung des Ausgleichssatzes ist so gering, daB keine Gemeinde zuweisungsberechtigt wird, die nicht schon vorher zuweisungen erhalten hätte.

Dann ist

$$
\sum_{i=n+1}^{n+k} \Delta s_{i}(\lambda)=0
$$


und daher auch

$$
\sum_{i=1}^{n} \Delta s_{i}(\lambda)=0 .
$$

Da aber für $i, j \in\{1, \ldots, n\}, i \neq j$

$$
\Delta s_{i} \neq \Delta s_{j} \neq 0 \leftrightarrow \bar{K}_{i} \neq \bar{k}_{j} \neq \frac{\sum_{i=1}^{n} k_{i}}{\sum_{i=1}^{n} E_{i} b_{i}}
$$

erfüllt ist, folgt in jedem Fall $\hat{n}<n$ und

$(6.3 .29)$

$$
\Delta s_{i}<0, i=1, \ldots, \hat{n}
$$

$$
\Delta s_{i}>0, i=\hat{n}+1, \ldots, n
$$

Die Umverteilung findet nun innerhalb der Gruppe $\{1, \ldots, n\}$ statt, wobei die reicheren Gemeinden auf Kosten der ärmeren besser gestellt werden.

Wir gehen nun ganz analog vor bei einer Erhöhung der Ausschüttungsquote.

1) Dies folgt sofort aus den Äquivalenzen

$$
\begin{aligned}
& \Delta S_{i}=0 \leftrightarrow \frac{\lambda G(\lambda a)-G(a)}{1-\lambda}=\bar{K}_{i} \\
& \sum_{i=1}^{n} \Delta S_{i}=0 \Leftrightarrow \frac{\lambda G(\lambda a)-G(a)}{1-\lambda}=\frac{\sum_{i=1}^{n} K_{i}}{\sum_{i=1}^{n} E_{i} b_{i}} .
\end{aligned}
$$




\subsubsection{Erhöhung der Ausschüttungsquote}

Sei $\lambda \in J_{n+k}, n \in\{1, \ldots, N\}$ und $\lambda>1$ fest mit

$(6.3 .30) \quad J_{n+k}= \begin{cases}{\left[\frac{\frac{a}{n+k}_{a}}{a}, \frac{\bar{a}_{n+k}}{a}\right),} & \bar{n}+1-n \leqq k \leqq 0 \\ {\left[\frac{a_{\bar{n}}}{a}, 1\right]} & , k=\bar{n}-n .\end{cases}$

Dann gibt $\mathrm{k}$ die $\mathrm{Zahl}$ der Gemeinden an, um die sich der Kreis der zuweisungsberechtigten Gemeinden verändert. Sie nimmt zumindest nicht $z u$, weil

$(6.3 .31) \quad \lambda>1 \Leftrightarrow k \leqq 0$

gilt.

Ein zu 6.3.2 analoges Vorgehen liefert

$(6 \cdot 3 \cdot 32)$

$\Delta S_{i}= \begin{cases}0 & , i=n+1, \ldots, N \\ -a\left(E_{i} b_{i} G(a)-k_{i}\right) & , i=n+k+1, \ldots, n \\ a E_{i} b_{i}(\lambda G(\lambda a)-G(a))+a(1-\lambda) k_{i}, i=1, \ldots, n+k\end{cases}$

Die Gemeinden $\{n+1, \ldots, N\}$ erhalten natürlich auch nach Erhöhung des Ausgleichssatzes keine Zuweisungen und $\Delta S_{i}=0$. Die Gemeinden $\{n+k+1, \ldots, n\}$ erhalten nun keine Zuweisungen mehr, $\Delta S_{i}$ entspricht bei ihnen daher genau dem negativen Zuweisungsbetrag, den sie beim Satz a noch erhalten hatten. Die Interpretation für die Gemeinden $\{1, \ldots, n+k\}$ ist wieder etwas komplizierter. Die Uberlegungen sind aber ähnlich wie in 6.3.2, weshalb unsere Darstellung etwas kürzer aus- 
fälıt. 1)

Da mit $k<0 \sum_{i=n+k+1}^{n} \Delta s_{i}<0$ ist, muB $\sum_{i=1}^{n+k} \Delta s_{i}>0$ sein, und es existiert ein $\tilde{n} \in\{1, \ldots, n+k\}$ mit

$$
\overline{\mathrm{K}}_{\tilde{\mathrm{n}}}<\frac{1}{1-\lambda}(\mathrm{G}(\mathrm{a})-\lambda \mathrm{G}(\lambda \mathrm{a})) \leqq \overline{\mathrm{K}}_{\tilde{\mathrm{n}}+1}
$$

und deshalb folgt mit $\bar{K}_{\tilde{n}+1} \leqq \bar{K}_{\tilde{n}+2}$

(6.3.34) $\quad \Delta s_{i} \begin{cases}>0 & , \quad i=1, \ldots, \tilde{n} \\ \leqq & , \quad i=\tilde{n}+1 \\ <0, & i=\tilde{n}+2, \ldots, n .\end{cases}$

Fassen wir die wichtigsten Ergebnisse des Abschnitts 6.3 nochmals zusammen:

Jede Veränderung des Ausgleichssatzes ist mit einer Umverteilung der Schlüsselmasse verbunden. Man kann daher keine Gemeinde besser stellen, ohne gleichzeitig mindestens eine andere Gemeinde schlechter zu stellen. Dabei bedeutet ein $\mathrm{zu}-$ wachs (bzw. ein Rückgang) der Schlüsselzuweisung bei irgendeiner Gemeinde eine ebenso große zunahme (bzw. Abnahme) der Finanzausstattung in dieser Gemeinde und gleichzeitig ein Anstieg (bzw. Rückgang) ihres Finanzanteils. Die Situation der steuerstärksten Gemeinden, also derjenigen, die vorher keine Schlüsselzuweisungen erhielten und auch nachher keine erhalten, ändert sich überhaupt nicht. Sie profitieren, was

1) Man beachte dazu, daß für $\lambda>1$ diesmal

$\Delta S_{i}>0 \leftrightarrow \frac{1}{1-\lambda}(G(a)-\lambda G(\lambda a))>\bar{K}_{i}$

und

$\frac{1}{1-\lambda}(G(a)-\lambda G(\lambda a))<G(\lambda a) \Leftrightarrow G(a)>G(\lambda a)$

gilt. 
wohl auch nicht erwünscht ist, deshalb nie von einer Umverteilung der Schlüsselmasse. Sie können aber auch, und dies ist aus der sicht der armen Gemeinden ein etwas trauriges Resultat, nie zugunsten dieser armen Kommunen schlechter gestellt werden. Umverteilung von reicheren Gemeinden (das sind dann aber nicht die reichsten) $z u$ ärmeren findet überhaupt nur statt, wenn der Ausgleichssatz angehoben wird. Dann werden in jedem Falle diejenigen Gemeinden schlechter gestellt, die aus dem Kreis der zuweisungsberechtigten Gemeinden ausscheiden, unter Umständen aber auch die reicheren Gemeinden unter denen, die auch nachher noch Zuweisungen erhalten. Eine Senkung des Ausgleichssatzes hat genau die umgekehrte Wirkung. Die armen Gemeinden müssen auf einen Teil ihrer Schlüsselzuweisungen verzichten, damit den neu hinzukommenden Gemeinden Zuweisungen gewährt werden können, unter Umständen werden damit aber auch die Zuweisungen der reicheren Gemeinden aus der ärmsten Gruppe erhöht.

\subsection{Progressive und regressive Verteilungen}

Wir zeigen nun, daß Verteilungen mit höherer Ausschüttungs quote progressiver sind als Verteilungen mit geringerer Ausschüttungsquote. Von irgendzwei Verteilungen

$$
\begin{aligned}
& \mathbf{s}:=\mathbf{s}(a)=\left(s_{1}(a), \ldots, s_{N}(a)\right) \text { und } \\
& \hat{\mathbf{s}}:=\mathbf{s}(\hat{a})=\left(s_{1}(\hat{a}), \ldots, s_{N}(\hat{a})\right)
\end{aligned}
$$

ist demnach $\hat{\mathbf{s}}$ progressiver als $\mathbf{s}$, wenn $\hat{a}>\mathrm{a}$ ist. Bezeichnet man mit $x=\left(x_{1}, \ldots, x_{N}\right)$ bzw. $y=\left(y_{1}, \ldots, y_{N}\right)$ die Verteilung der normierten Finanzausstattungen bei $\hat{\mathbf{s}}$ bzw. $s$ und mit 
(6.4.1) $\quad x_{i}:=\frac{\bar{F}_{i}(\hat{a})}{\sum_{j=1}^{N} \bar{F}_{j}(\hat{a})} \quad$ bzw. $\quad y_{i}:=\frac{\bar{F}_{i}(a)}{\sum_{j=1}^{N} \bar{F}_{j}(a)}$

den normierten Finanzanteil von Gemeinde $i$ bei $\hat{s}$ bzw. s, dann muB $\mathrm{XL}_{\mathrm{P}} \mathrm{Y}$ gelten.

Dazu zeigen wir (siehe $(3.3 .6),(3.3 .7))$ :

a) $x_{1} \leqq x_{2} \leqq \cdots \leqq x_{N} \Rightarrow y_{1} \leqq y_{2} \leqq \cdots \leqq y_{N}$

b) Es existiert ein $i_{0} \in\{1, \ldots, N-1\}$ mit

$$
\begin{array}{ll}
y_{i} \leqq x_{i} \quad, \quad i=1, \ldots, i_{0} \\
y_{i} \geqq x_{i} \quad, \quad i=i_{0}+1, \ldots, N
\end{array}
$$

und zusätzlich

$$
\mathrm{y}_{1}<\mathrm{x}_{1} \wedge \mathrm{y}_{\mathrm{N}}>\mathrm{x}_{\mathrm{N}}
$$

ad a) Es gilt:

$$
\begin{aligned}
& \left\{x_{1} \leqq x_{2} \leqq \cdots \leqq x_{N} \Rightarrow y_{1} \leqq y_{2} \leqq \cdots \leqq y_{N}\right\} \\
& \Leftrightarrow\left\{\begin{array}{l}
\bar{F}_{1}(\hat{a}) \leqq \bar{F}_{2}(\hat{a}) \leqq \cdots \leqq \bar{F}_{N}(\hat{a}) \Rightarrow \\
\bar{F}_{1}(a) \leqq \bar{F}_{2}(a) \leqq \cdots \leqq \bar{F}_{N}(a)
\end{array}\right\}
\end{aligned}
$$

In 4.2 wurde gezeigt, $d a \beta$ bei jeder Verteilung der Schlüsselzuweisungen die ordnung der normierten Finanzausstattungen mit der ordnung der normierten Steuerkraft übereinstimmt. 
ad b)

Bezeichne:

$$
\bar{F}(a):=\sum_{i=1}^{N} \bar{F}_{i}(a), \quad a \in(0,1]
$$

(6.4.2) $\quad \Delta \bar{s}_{i}:=\bar{S}_{i}(\hat{a})-\bar{s}_{i}(a)$

$$
\Delta \bar{S}:=\sum_{i=1}^{N} \Delta \bar{S}_{i}
$$

Dann gilt allgemein mit der Annahme $\Delta \bar{S}>0$ (wird später überprüft):

(6.4.3) $\quad x_{i}>y_{i} \leftrightarrow \frac{\bar{F}_{i}(\hat{a})}{\bar{F}(a)} \geqq \frac{\bar{F}_{i}(a)}{\bar{F}(a)} \cdot \frac{\Delta \bar{S}_{i}}{\Delta \bar{S}} \geqq \frac{\bar{F}_{i}(a)}{\bar{F}(a)}$.

Sei â:= $\lambda$ a mit $\underline{\lambda}>1$ und seien a und $\lambda$ (fest) wie in Kapitel 6.3 spezifiziert. Dann kann man (6.3.32) die Werte $\Delta \bar{S}_{i}:=\Delta \bar{S}_{i}(\lambda)$ für $i=1, \ldots, N$ entnehmen.

Damit gilt in den Gemeinden

$i=n+1, \ldots, N$ :

(6.4.4) $\quad \Delta \bar{S}_{i}=0 \Rightarrow x_{i}<y_{i}$

$i=n+k+1, \ldots, n:$

(6.4.5) $\Delta \bar{S}_{i}<0 \Rightarrow x_{i}<y_{i}$.

Damit gibt es unter den Gemeinden $\{1, \ldots, n+k\}$ mindestens ein $i \operatorname{mit} x_{i}>y_{i}$. 
$i=1, \ldots, \mathrm{n}+\mathrm{k}:$

Wir wissen, es existiert ein $\tilde{n} \in\{1, \ldots, n+k\}$ mit

(6.4.6) $\quad \Delta s_{i} \leqq 0, \quad i=\tilde{n}+1, \ldots, n$.

Dabei ist $\tilde{\mathrm{n}}$ durch $(6.3 .33)$ spezifiziert und

$$
\Rightarrow x_{i}<y_{i}, i=\tilde{n}+1, \ldots, n .
$$

Für $i=1, \ldots, \tilde{n}:$

(6.4.7) $\quad x_{i}>y_{i} \leftrightarrow \bar{K}_{i}<\frac{\bar{F}(a)(\lambda G(\lambda a)-G(a))-\Delta \bar{S} G(a)}{(1 / a-1) \Delta \bar{S}-(1-\lambda)}=: D$.

Es existiert also ein $\hat{n} \in\{1, \ldots, \tilde{n}\}$ mit

$$
\begin{array}{lll} 
& K_{\hat{n}}<D \leqq K_{\hat{n}+1}, & \hat{n} \in\{1, \ldots, \tilde{n}-1\} \\
(6.4 .8) & K_{\tilde{n}}<D & , \hat{n}=\tilde{n}
\end{array}
$$

und

$$
\text { (6.4.9) } \quad x_{i}>y_{i} \quad, \quad i=1, \ldots, \hat{n} .
$$

\section{Insgesamt gilt}

$\left.\begin{array}{rl}x_{i} & <y_{i}, \quad i=\tilde{n}+1, \ldots, N \\ (6.4 .10) \quad x_{i} & \leqq y_{i}, i=\hat{n}+1, \ldots, \tilde{n} \\ x_{i} & >y_{i}, i=1, \ldots, \hat{n}\end{array}\right\} \Rightarrow x_{p} y$. 
Abschließend überprüfen wir noch die Annahme $\Delta \bar{S}>0$.

Es gilt:

$$
\begin{aligned}
& \Delta \bar{s}>0--a \sum_{n+k+1}^{n}\left(G(a)-\bar{K}_{i}\right)+a \sum_{i=1}^{n+k}\left(\lambda G(\lambda a)-G(a)+(1-\lambda) \bar{K}_{i}\right)>0 \\
& -\lambda\left((n+k) G(\lambda a)-\sum_{i=1}^{n+k} \bar{K}_{i}\right)>G(a)(2 k+n)-\sum_{i=1}^{n} \bar{K}_{i} \\
& -\lambda>\frac{G(a)(2 k+n)-\sum_{i=1}^{n} \bar{K}_{i}}{(n+k) G(\lambda a)-\sum_{i=1}^{n+k} \bar{K}_{i}},
\end{aligned}
$$

da mit $\overline{\mathrm{K}}_{i}<G(\lambda a), i=1, \ldots, \mathrm{n}+\mathrm{k}$

$$
-\sum_{i=1}^{n+k} \bar{K}_{i}+(n+k) G(\lambda a)>0
$$

ist. Mit der Annahme $\lambda>1$ muß gelten:

$$
G(a)(2 k+n)-\sum_{i=1}^{n} \bar{K}_{i} \geqq(n+k) G(\lambda a)-\sum_{i=1}^{n+k} \bar{K}_{i}
$$

$$
\text { - } k G(a)+(k+n)(G(a)-G(\lambda a))>\sum_{i=n+k+1}^{n} \bar{K}_{i} .
$$

Mit $G(a)>\bar{K}_{i}, i=1, \ldots, n \Rightarrow k G(a)>\sum_{i=n+k+1}^{n} \bar{K}_{i}$ und mit $G(a)>G(\lambda a)$ folgt obige Beziehung. 
7 Analyse der Sonderschlüsselzuweisungen und Mindestgaran$\underline{\text { tien }}$

Bisher haben wir von Sonderschlüsselzuweisungen und Mindestgarantien abstrahiert. Sie werden nun in unsere Analyse einbezogen.

Dazu gehen wir von einer beliebigen Verteilung $\left(S_{1}^{s} \ldots \ldots, s_{N}^{s}\right)$ der Sonderschlüsselzuweisungen aus, von der nur

$$
s_{i}^{s} \geqq 0, i=1, \ldots, N \text { und }
$$

$$
s_{i}^{s}>0 \text { für mindestens ein } i
$$

gefordert ist. ${ }^{1)}$ Für schlüsselzuweisungen und Sonderschlüsselzuweisungen stehe wieder die (exogene) Finanzmasse Mg zur Verfügung. Beim Vorwegabzug sind die Sonderschlüsselzuweisungen bekanntlich von Mg unabhängig. Für Schlüsselzuweisungen verbleibt also die Schlüsselmasse

$$
M=M g-\sum_{i=1}^{N} s_{i}^{s}
$$

wobei noch $\mathrm{Mg}>\sum_{i=1}^{N} \mathrm{~S}_{i}^{\mathrm{s}}$ und folglich $\mathrm{M}>0$ vorausgesetzt sei. Die Verteilung der Schlüsselzuweisungen

$$
s(M)=\left(S_{1}(M), \ldots, S_{N}(M)\right)
$$

ist eindeutig bestimmt.

Die Finanzausstattung $F_{i}^{S}$ der Gemeinde $i$ mit Sonderschlüssel-

1) Eine Spezifikation dieser Verteilungen erhält man, wenn man die gesetzliche Regelung eines Landes zugrunde legt, vgl. 2.2. 


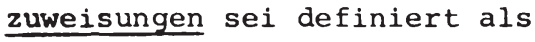

(7.3)

$$
F_{i}^{s}(M):=K_{i}+s_{i}(M)+s_{i}^{s}
$$

und

$$
\mathbf{x}=\left(\overline{\mathrm{F}}_{1}^{\mathbf{S}}(\mathrm{M}), \ldots, \overline{\mathrm{F}}_{\mathrm{N}}^{\mathbf{S}}(\mathrm{M})\right)
$$

sei eine Verteilung der normierten Finanzausstattungen mit Sonderschlüsselzuweisungen, wobe $i$

$$
\bar{F}_{i}^{s}(M):=\frac{F_{i}^{8}(M)}{E_{i} b_{i}}, i=1, \ldots, N
$$

vereinbart sei.

Die Situation bei dieser Verteilung vergleichen wir nun mit einer Situation, in der es Sonderschlüsselzuweisungen nicht gibt und in der die gesamten Finanzmittel Mg nur für Schlüsselzuweisungen ausgegeben werden. Dann erhält man die (eindeutige) Verteilung der Schlüsselzuweisungen

$$
s(M g)=\left(s_{1}(M g) \ldots s_{N}(M g)\right)
$$

und die Finanzausstattung der Gemeinden (ohne Sonderschlüsselzuweisungen) wie bisher als

$$
F_{i}(M g)=k_{i}+s_{i}(M g), i=1, \ldots, N
$$

Daraus resultiert die Verteilung der normierten Finanzausstattungen

$$
(7.8) \quad y=\left(\bar{F}_{1}(M g), \ldots, \bar{F}_{N}(M g)\right)
$$

mit

$$
\bar{F}_{i}(M g)=\frac{F_{i}(M g)}{E_{i} b_{i}}, i=1, \ldots, N
$$


die wir mit der Verteilung $x$ vergleichen wollen.

Es gilt:

$$
\bar{F}_{i}^{S}(M)>\bar{F}_{i}(M g) \leftrightarrow \bar{S}_{i}(M)+\bar{S}_{i}^{S}>\bar{S}_{i}(M g)
$$

$$
\rightarrow \overline{\mathbf{S}}_{i}^{\mathbf{s}}>\Delta \overline{\mathrm{S}}_{i},
$$

wobei $\overline{\Delta S}_{1}:=\bar{S}_{1}(M g)-\bar{S}_{i}(M)$ ist.

Eine Gemeinde ist bei der Verteilung $x$ also besser gestellt als bei $y$, wenn ihre Sonderschlüsselzuweisung gröBer ist als die Differenz zwischen ihrer Schlüsselzuweisung bei Mg und ihrer Zuweisung bei $M$.

Wenn eine Gemeinde $i_{0} \in\{1, \ldots, N-1\}$ existiert mit

(7.10) $\begin{cases}\bar{s}_{i}^{s} \geqq \Delta \bar{s}_{i}, & i=1, \ldots, i_{0} \\ \bar{s}_{i}^{s} \leqq \Delta \bar{s}_{i}, & i=i_{0}+1, \ldots, N\end{cases}$

und wenn

$$
x_{1} \leqq \cdots \leqq x_{N} \Rightarrow y_{1} \leqq \cdots \leqq y_{N}
$$

gilt, dann ist $x$ mindestens so gleich wie $y$ (vgl. (3.3.6)).

Ob die Bedingung (7.10) erfüllt ist, kann man nicht allgemein sagen. Zwar sind die Größen $\Delta \overline{\mathrm{S}}_{i}$ eindeutig bestimmt (siehe 5.3), die Sonderschlüsselzuweisungen sind aber nur spezifiziert, wenn die gesetzliche Regelung in einem bestimmten Land zugrunde gelegt wird.

Trotzdem läßt eine einfache Uberlegung die Vermutung $z u$, daß $x$ im allgemeinen gleicher sein wird als $y$. Man kann nämlich leicht zeigen, daß die verfügbaren Finanzmittel, die 
bekanntlich bei $x$ für Sonderschlüsselzuweisungen und be $i \mathrm{y}$ für zusätzliche Schlüsselzuweisungen Verwendung finden, bei $y$ auf wesentlich mehr (und zum Teil reichere Gemeinden) verteilt werden als bei $x$. So erhalten bei $y$ Gemeinden Schlüsselzuweisungen, die bei $x$ nicht zuweisungsberechtigt sind und erst recht keine Sonderschlüsselzuweisungen bekommen.

Nehmen wir also an:

$$
\begin{aligned}
& s_{i}^{s}>0, i=1, \ldots, m \\
& s_{i}(M)>0, \quad i=1, \ldots, n(M) \\
& s_{i}(M g)>0, \quad i=1, \ldots, n(M g) .
\end{aligned}
$$

Meist ist $m<n(M)$ und wegen $M<M g$ ist $n(M) \leqq n(M g)$, meist sogar $n(M)<n(M g)$, falls zusätzlich

$$
\Delta M:=\sum_{i=1}^{N} S_{i}^{S}=M g-M
$$

so groß ist, daB Intervallgrenzen überschritten werden (siehe 5.1), wovon man aber ausgehen kann. Daraus folgt

$$
\begin{aligned}
& \Delta s_{i}=0, \quad i=n(M g)+1, \ldots, N \\
& \Delta s_{i}>0, \quad i=1, \ldots, n(M g),
\end{aligned}
$$

wenn wir noch die strenge Monotonie der Schlüsselzuweisungen beachten.

Es gilt also:

$$
\begin{aligned}
& s_{i}^{s}=\Delta s_{i}=0, \quad i=n(M g)+1, \ldots, N \\
& \Delta s_{i}>s_{i}^{s}=0, \quad i=m+1, \ldots, n(M g) .
\end{aligned}
$$


Damit gibt es unter den Gemeinden $\{1, \ldots, m\}$ wegen $\sum_{i=1}^{m} s_{i}^{s}=\sum_{i=1}^{n}(M g) \Delta s_{i}$ mindestens ein $i$ mit

$$
s_{i}^{s}>\Delta s_{i}
$$

Die Sonderschlüsselzuweisungen, die das System nur weiter komplizieren, könnten trotz ihrer nivellierenden Wirkung überflüssig werden, wenn man dafür bei den Schlüsselzuweisungen den Ausgleichssatz erhöht. Denn falls Sonderschlüsselzuweisungen fiktiv als Mindestgarantien gewährt werden, so ist ein Ausgleichssatz von $a=1$ einem Garantiesatz von $a_{s}=1$ (wie wir in 2.2 .2 erkannt haben) gleichwertig, d.h. er erzeugt die gleiche Verteilung. Präferiert man Mindestgarantien mit einem Garantiesatz von $a_{s}<1$, dann läßt sich die resultierende Verteilung auch ohne Mindestgarantien erzeugen, wenn'man den Ausgleichssatz nach Gemeinden differenziert.

Dazu gehen wir von einer Verteilung

$$
s^{g}=\left(s_{1}^{g}, \ldots, s_{N}^{g}\right)
$$

aus, in der die Schlüsselzuweisungen durch

(2.2.11) $s_{i}^{g}= \begin{cases}a_{s} E_{i} b_{i} G-k_{i} & \Psi_{i}=1 \\ \theta_{i} a\left(E_{i} b_{i} G-k_{i}\right), & \Psi_{i}=0\end{cases}$

bestimmt sind, also Mindestgarantien enthalten (siehe 2.2.2). Die zugehörigen Dummy-Variablen $\Psi_{i}$ bzw. $\theta_{i}$ sind durch (2.2.7) bzw. (2.2.2) spezifiziert. Insbesondere folgt mit
$(2.2 .9)$
$\Psi_{i}=1$
$\Rightarrow \theta_{i}=1$
und
$(2.2 \cdot 10)$
$\theta_{i}=0 \Rightarrow \Psi_{i}=0$ 
aus $(2.2 .11)$

$$
s_{i}^{g} \begin{cases}=0, & \theta_{i}=0 \\ >0, & \theta_{i}=1 \vee \Psi_{i}=1 .\end{cases}
$$

Der zugehörige Grundbetrag $G$ ist durch (2.2.13) so bestimmt, daB die Bedingung

$$
\sum_{i=1}^{N} s_{i}^{g}=M g
$$

erfüllt ist.

Gesucht ist nun eine $\mathrm{zu} \mathrm{s}^{\mathrm{g}}$ identische Verteilung $\widetilde{s}=\left(\tilde{S}_{1}, \ldots, \tilde{S}_{N}\right)$, die ohne Mindestgarantien auskommt. Es soll also

$(7.13)$

$$
\widetilde{s}_{i}=s_{i}^{g} \quad, \quad i=1, \ldots, N
$$

gelten.

Man wähle Schlüsselzuweisungen mit differenzierten Ausschüttungsquoten $a_{i}, i=1, \ldots, N$

$$
\widetilde{S}_{i}=\theta_{i} a_{i}\left(E_{i} b_{i} G-K_{i}\right), \quad i=1, \ldots, N \text {, }
$$

wobei der Grundbetrag $G$ mit dem Grundbetrag von $s^{g}$ identisch sei. Dann sind auch die Dummy-Variablen $\theta_{i}$ wie bei $s^{g}$ spezifiziert, und es gilt:

(7.15) $\left\{\begin{array}{l}s_{i}^{g}=\widetilde{s}_{i}=0, \theta_{i}=0 \\ s_{i}^{g}>0, \widetilde{s}_{i}>0, \theta_{i}=1 .\end{array}\right.$

Nun kann man $a_{i}(i=1, \ldots, N)$ geeignet festsetzen, so da $\beta$ (7.13) erfüllt ist: 
für $\Theta_{i}=0: a_{i}$ beliebig,

für $\Theta_{i}=1$ erhält man durch Einsetzen von (7.13) in (7.14)

$(7.16)$

$$
a_{i}=\frac{s_{i}^{g}}{E_{i} b_{i} G-k_{i}} \text {. }
$$

Setzt man (2.2.11) ein, so folgt

(7.17) $\quad a_{i}= \begin{cases}a & \Psi_{i}=0 \\ \frac{a_{s} E_{i} b_{i} G-K_{i}}{E_{i} b_{i} G-K_{i}}, & \Psi_{i}=1 .\end{cases}$

Die Ausschüttungsquoten müssen also nur für Gemeinden differenziert werden, bei denen die Mindestgarantie wirksam wird. Die Ausschüttungsquote $a_{i}$ einer Gemeinde $i$ mit $\Psi_{i}=1$ ist stets größer als $a$, und sie ist umso höher, je ärmer die Gemeinde ist, denn es gilt

$$
a_{i} \geqq a_{j} \leftrightarrow \bar{k}_{i} \leqq \bar{k}_{j} \quad, i, j \in\left\{k \mid \Psi_{k}=1\right\} .
$$




\section{I I KRITIK UND REFORMVORSCHLÄGE}

\section{Grenzen der Redistribution}

In diesem Kapitel wollen wir noch etwas genauer zeigen, wie die redistributiven Möglichkeiten im System der Schlüsselzuweisungen sehr stark von der Schlüsselmasse eingeschränkt werden und uns dazu auch auf Erkenntnisse aus früheren Kapiteln stützen.

Wie wir wissen, findet bei einer Schlüsselmasse von 0 keine Umverteilung statt. Damit ist über die Progressivität des Systems schon sehr viel ausgesagt, denn es gibt quasi eine Besitzstandsgarantie: Keine Kommune muB von ihren Steuereinnahmen zur Finanzierung der Zuweisungen an bedürftige Kommunen etwas abgeben. Redistribution ist mit Schlüsselzuweisungen also erst dann möglich, wenn die $z$ u verteilenden Finanzmittel von den Gemeinden nicht vorher selbst aufgebracht werden müssen, sondern vom Land bereitgestellt werden. Was diesem System völlig fehlt, ist die Möglichkeit, Umlagen zu erheben. Zwar muß man diese Aussage noch etwas abschwächen, denn im Grunde wird auch die Schlüsselmasse letztlich aus dem Gemeindeanteil an der Verbundmasse gespeist, also gewissermaßen aus Mitteln, die den Gemeinden insgesamt an den Gemeinschaftssteuern zur Verfügung stehen. Dennoch macht es einen Unterschied, ob die Schlüsselmasse über Umlagen, also aus Gemeindesteuern, oder aus dem Steuerverbund finanziert wird. Denn wir werden gleich sehen, daß fiskalische Gleichheit mit dem derzeitigen System nur sehr schwer, wenn überhaupt, erreicht werden kann. Die Besitzstandsgarantie beläBt eben den reichen Gemeinden ihre hohen Steuereinnahmen, so daß die Schlüsselmasse bei weitem nicht ausreicht, die Finanzausstattung der ärmeren Gemeinden auf dieses Niveau anzuheben. Für die redistributiven Möglichkeiten, die bei Schlüsselzuweisungen noch verbleiben, hat dies natürlich 
erhebliche Konsequenzen. Betrachten wir den ganzen Mechanismus noch etwas genauer.

Wie wir wissen, kann fiskalische Ungleichheit durch die Erhöhung der Schlüsselmasse oder der Ausschüttungsquote reduziert werden. Bei niedrigen Schlüsselmassen ist auch das Versorgungsniveau relativ niedrig. Die fiskalische Ungleichheit der Kommunen rührt dann im wesentlichen daher, daß es bei diesem Versorgungsniveau viele abundanten Gemeinden mit Finanzierungsüberschüssen gibt, während die zuweisungsberechtigten Gemeinden bei Ausschüttungsquoten gröBer als 1 noch Finanzierungsdefizite aufweisen. Hinzu kommt, daß auch unter den abundanten Gemeinden selbst erhebliche fiskalische Unterschiede bestehen können.

Durch die Erhöhung der Schlüsselmasse wird eine größere Zahl von Kommunen zuweisungsberechtigt und die fiskalische Ungleichheit verringert sich. Nun wissen wir, daB es für fiskalische Gleichheit notwendig wäre, das Versorgungsniveau so weit anzuheben, bis alle Gemeinden zuweisungsberechtigt werden. Dies allein würde aber noch nicht ausreichen, denn auch die Ausschüttungsquote muß bis auf 1 erhöht werden. Eine steigende Ausschüttungsquote reduziert zwar die fiskalische Ungleichheit, bei gleichen Schlüsselmassen würde jedoch das Versorgungsniveau und die Zahl der zuweisungsberechtigten Gemeinden wieder abnehmen. Dies kann nur durch eine weitere Erhöhung der Schlüsselmasse kompensiert werden. Dahinter verbirgt sich die einfache Regel, daß höhere Ausschüttungsquoten erst finanziert werden müssen, d.h. höhere Schlüsselmassen erforderlich machen. Das läßt sich sehr leicht einsehen.

Betrachten wir nochmals die Gleichung 
(2.3.3) $\quad \sum_{i=1}^{N} \Theta_{1} E_{i} b_{i}\left(G-\bar{K}_{i}\right)=\frac{M}{a}$,

die für eine bestimmte Schlüsselmasse M und eine bestimmte Ausschüttungsquote a bekanntlich einen eindeutigen Grundbetrag $G(M, a)$ als Lösung hat $(s .2 .3)$. Man erkennt sofort, daß

$$
G(M, a)=G(\lambda M, \lambda a), \text { für } \lambda>0
$$

und folglich

$$
\theta_{i}:=\theta_{i}(M, a)=\theta_{i}(\lambda M, \lambda a), \quad \lambda>0, \quad i=1, \ldots, N
$$

ist. Für die Schlüsselzuweisungen folgt daraus

$(8.3)$

$$
S_{i}(\lambda M, \lambda a)=\lambda S_{i}(M, a), \quad \Theta_{i}=1
$$

$$
S_{i}(\lambda M, \lambda a)=S_{i}(M, a)=0, \theta_{i}=0 \text {. }
$$

Man sieht, daß zusätzliche Finanzmittel hier ausschließlich auf die Gemeinden verteilt werden, die vorher schon zuweisungsberechtigt gewesen sind. Ihre Schlüsselzuweisungen steigen um das $\lambda$-fache, wenn die Ausschüttungsquote um den gleichen Faktor steigt.

Dieser einfache Fall zeigt, daß die Strategie, zur Beseitigung der fiskalischen Ungleichheit gleichzeitig das Versorgungsniveau und die Ausschüttungsquote anzuheben, eigentlich nur eine theoretische Möglichkeit darstellt. Denn dafür wären Schlüsselmassen in einer Höhe erforderlich, wie sie in der Realität wohl nie zu erreichen sein dürfte. Wir können übrigens den benötigten Betrag genau angeben (siehe 6.1). Die in den letzten Jahren tatsächlich vorhandenen 
Schlüsselmassen hätten jedenfalls dafür bei weitem nicht ausgereicht.

Unabhängig davon ist zu fragen, ob es überhaupt eine sinnvolle Konstruktion sein kann, das Versorgungsniveau aus Verteilungsgesichtspunkten heraus an der steuerkraft der steuerstärksten Kommune auszurichten. Denn genau das wäre die logische Konsequenz, wenn man mit Schlüsselzuweisungen fiskalische Gleichheit erzeugen wollte. In der Praxis jedenfalls ist es nicht möglich, alle Gemeinden so auszustatten wie die steuerstärksten. Dafür reichen die finanziellen Möglichkeiten einfach nicht aus. Gerade die reichen Kommunen haben Zuweisungen auch gar nicht nötig, bei einem realistischen Versorgungsniveau müBten sie vielmehr in der Lage sein, von ihren Finanzierungsüberschüssen an arme Gemeinden etwas abzugeben. Diese Möglichkeit fehlt bei den Schlüsselzuweisungen aber ganz.

Das System erzeugt dagegen eine Diskrepanz zwischen der vorhandenen Schlüsselmasse und derjenigen, die man für fiskalische Gleichheit benötigen würde. Deshalb wird man damit fiskalische Ungleichheit praktisch nie beseitigen können. So befindet man sich in einem Dilemma, aus dem es innerhalb des Systems keinen Ausweg gibt: Einerseits kann fiskalische Ungleichheit nicht unabhängig von der Schlüsselmasse reguliert werden, andererseits eignet sich diese Variable aufgrund exogener Beschränkungen kaum, oder jedenfalls nur sehr bedingt, als Regulativ.

Die systemimmanenten Restriktionen können eigentlich nur überwunden werden, wenn man ein Umlageverfahren einführt ${ }^{1}$ ),

1) Umlagen schlägt z.B. auch Seiler [1980], S. 66, vor. 
das die Finanzierungsüberschüsse der reichen Gemeinden abschöpft. Wie ein solches system beschaffen sein müBte, wollen wir uns im nächsten Kapitel genauer ansehen.

Bei der gegenwärtigen Regelung muß man sich darauf beschränken, die fiskalische Ungleichheit zu reduzieren. Das ist am ehesten mit einer Erhöhung der Ausschüttungsquote möglich. Bei konstanter Schlüsselmasse verteilen sich die Finanzmittel damit auf weniger (und ärmere) Kommunen, von denen jede absolut und relativ mehr erhält. Die Schlüsselmasse ist als Instrumentvariable dagegen weitgehend ungeeignet. Sie ist nach oben nur variabel, wenn die Steuereinnahmen steigen. Dann sollten höhere Schlüsselmassen allerdings nicht für die Erweiterung des Kreises zuweisungsberechtigter Gemeinden, sondern zur Finanzierung höherer Ausschüttungsquoten verwendet werden, weil sich damit die fiskalische Ungleichheit noch weiter reduzieren ließe.

Bei einer steigenden Schlüsselmasse befindet man sich im System der Schlüsselzuweisungen noch in einer vergleichsweise günstigen Lage. Fast aussichtslos sieht man sich jedoch einer Situation gegenüber, in der die Schlüsselmasse, etwa in zeiten zurückgehender Steuereinnahmen, sinkt. Dann nimmt unter sonst gleichen Bedingungen auch die fiskalische Ungleichheit wegen ihrer Abhängigkeit von der Schlüsselmasse (ungewollt) zu. Dies könnte durch eine entsprechende Erhöhung der Ausschüttungsquote entweder teilweise oder ganz kompensiert werden. Das system bietet jedoch starke Anreize, sich anders zu verhalten, als wir es eben empfohlen haben. Dies liegt daran, daß eine Reduktion der fiskalischen Ungleichheit auf den politischen widerstand insbesondere derjenigen Kommunen stößt, die dabei aus dem Kreis der $\mathrm{Zu-}$ weisungsempfänger herausfallen würden ${ }^{1)}$.

1) Wie wir wissen, sind die EinbuBen dieser Gemeinden in Wirklichkeit jedoch geringer als die der ärmeren. 
So könnte man versucht sein, eine sinkende Schlüsselmasse mit einer um den gleichen Faktor gesenkten Ausschüttungsquote zu beantworten. Der Kreis der zuweisungsberechtigten Kommunen würde sich dadurch, wie man an obigem Beispiel leicht sieht, nicht verändern. Das Versorgungsniveau wäre das gleiche, jede Kommune würde nur einen geringeren Prozentsatz ihres Finanzierungsdefizits als zuweisung erhalten. Da dies alle zuweisungsberechtigten Gemeinden trifft, und die abundanten Kommunen auch nachher keine Zuweisungen erhalten, könnte man fast glauben (und so ließe sich im politischen Bereich sicher auch argumentieren), daB keine Gemeinde relativ schlechter gestellt wird. In Wirklichkeit ist die fiskalische Ungleichheit in diesem Fall, wie wir wissen, sogar noch höher als die, die allein durch die sinkende Schlüsselmasse hervorgerufen wird. Und dies würde insbesondere die ärmsten Kommunen treffen.

Wir betrachten das Ganze jetzt noch aus der Sicht der Gemeinden. Wenn die Gemeinden über die Schlüsselmasse und die Ausschüttungsquote selbst bestimmen könnten, dann würde jede Gemeinde natürlich ihre optimale Schlüsselmasse und ihre optimale Ausschüttungsquote wählen. Die zugehörigen optimalen Verteilungen der Kommunen unterscheiden sich sehr stark, weil eine Kommune ihren Finanzanteil immer nur auf Kosten der anderen Kommunen maximieren kann. Wie wir wissen, präferieren die armen Gemeinden Verteilungen mit relativ hohen Schlüsselmassen und hohen Ausgleichssätzen, und die reichen Verteilungen mit relativ niedrigen Schlüsselmassen und niedrigen Ausschüttungsquoten. Nun ist es jedoch so, daB all diese Uberlegungen nur theoretischer Natur sind, denn es kann in jedem Jahr nur eine Verteilung realisiert werden. Sie richtet sich in der Regel auch nicht nach den Interessen einzelner Kommunen, sondern es ist eine Verteilung, die aus den gesetzlichen Bestimmungen resultiert und mit der der staat seine eigenen ziele verwirklichen 
möchte. Aus der Sicht einer Kommune weicht diese Verteilung. natürlich mehr oder weniger von der Verteilung ab, die für sie optimal wäre. Welche Kommunen ihrem optimalzustand relativ nahe kommen, und welche davon weit entfernt sind, kann man nicht allgemein sagen. Dazu müßte man in jedem einzelnen Fall die optimale Schlüsselmasse und die optimale Ausschüttungsquote mit der verfügbaren Schlüsselmasse und der gesetzlich festgelegten Ausschüttungsquote vergleichen. Sicherlich gehen die Interessen der armen Gemeinden und des Staates in die gleiche Richtung, denn beide präferieren hohe Schlüsselmassen und hohe Ausgleichssätze. In der Realität dürften die armen Gemeinden allerdings immer noch stark benachteiligt sein, weil die gesetzlichen Ausgleichssätze und die verfügbaren Schlüsselmassen wesentlich niedriger sind, als sie sich staat und arme Gemeinden eigentlich wünschen. 


\section{Ein alternatives System von Zuweisungen}

Wir betrachten im folgenden ein System von zuweisungen, mit dem sich, ganz anders als bei den Schlüsselzuweisungen, fiskalische Gleichheit bei jeder beliebigen Schlüsselmasse erzeugen läßt.

Sei also $\underline{z}_{i}$ die Zuweisung an die Gemeinde $i, i=1, \ldots, N$ in diesem System und $z=\left(z_{1}, \ldots, z_{N}\right)$ eine Verteilung der Zuweisungen, von der nur verlangt wird, daß sie die exogene Schlüsselmasse $M \geqq 0$ (die also auch Null sein kann) ausschöpft:

$$
M=\sum_{i=1}^{N} z_{i}
$$

Die Finanzausstattung einer Gemeinde $i$ ist wie üblich definiert als

$$
F_{i}:=z_{i}+k_{i} \quad, i=1, \ldots, N_{1}
$$

und das Finanzierungsdefizit als

$$
D_{i}:=B_{i}-F_{i} \quad, i=1, \ldots, N
$$

Dabei berechnet sich der Finanzbedarf $B_{i}$ einer Gemeinde $i$ als

$$
B_{i}:=E_{i} b_{i} v
$$

$E_{i}$ sei ihre Einwohnerzahl, $b_{i}$ der Wert eines Bedarfsindex und $v$ ein für alle Gemeinden einheitliches Versorgungsniveau.

Die Zuweisungen $\mathrm{z}_{1}, \ldots, \mathrm{z}_{\mathrm{N}}$ sind nun so $\mathrm{zu}$ spezifizieren, daß fiskalische Gleichheit (s. (3.2.9)) 
$(9.5)$

$$
\begin{aligned}
& \bar{D}_{i}=0, \quad i=1, \ldots, N \\
& \text { mit } \bar{D}_{i}:=\frac{D_{i}}{E_{i} b_{i}}
\end{aligned}
$$

ermöglicht wird.

Die Bedingung (9.5) ist erfüllt, wenn die Zuweisungen die Form

$$
z_{i}=B_{i}-k_{i} \quad, i=1, \ldots, N
$$

haben und das Versorgungsniveau durch

$$
v=\frac{M+\sum_{i=1}^{N} K_{i}}{\sum_{i=1}^{N} E_{i} b_{i}}
$$

bestimmt ist. ") Dann ist, wie man leicht zeigt, die Schlüsselmasse $M$ ausgeschöpft, und es gilt

$$
F_{i}=B_{i}, i=1, \ldots, N \text {. }
$$

Daraus folgt mit $\bar{F}_{i}:=F_{i} / E_{i} b_{i}$

(9.8) $\bar{F}_{i}=v$ und $\bar{D}_{i}=0$ für alle $i=1, \ldots, N$.

Alle Gemeinden können mit ihren finanziellen Mitteln also genau den durchschnittlichen Bedarf decken. Dieser Bedarf bemiBt sich nach den finanziellen Möglichkeiten, denn das

1) Man beachte, das Versorgungsniveau ist im Gegensatz zum Grundbetrag explizit gegeben und kann also (im voraus) sehr einfach mit (9.7) berechnet werden, wenn man $M, K_{i}, E_{i} b_{i}, i=1, \ldots, N$ einsetzt. 
Versorgungsniveau kann man auch interpretieren als die für einen landesdurchschnittlichen Einwohner zur Verfügung stehenden Mittel (vgl. (9.7)). Man beachte, fiskalische Gleichheit ist nur realisierbar, weil reiche Gemeinden Umlagen ${ }^{1}$ aufbringen, denn für

$$
B_{i}<K_{i} \leftrightarrow v<\bar{K}_{i}
$$

ist $Z_{i}<0$. Bei einer Schlüsselmasse $M=0$ gibt es sicher mindestens eine Gemeinde $i$ mit $z_{i}<0$.

Ist fiskalische Gleichheit nicht erwünscht, so könnte man, wie bei den Schlüsselzuweisungen

$$
z_{i}=a\left(B_{i}-K_{i}\right), 0<a<1
$$

fordern. Reiche Gemeinden müssen nur a-Prozent ihres Steuerkraftüberschusses abgeben, arme Gemeinden erhalten nur a-Prozent ihres Bedarfsüberschusses. Das Versorgungsniveau ist dann durch

$(9.10)$

$$
v=\frac{\frac{M}{a}+\sum_{i=1}^{N} k_{i}}{\sum_{i=1}^{N} E_{i} b_{i}}
$$

gegeben. Die normierte Finanzausstattung einer Gemeinde

$$
\overline{\mathrm{F}}_{i}=a v+(1-a) \overline{\mathrm{K}}_{i} \quad, \quad i=1, \ldots, \mathrm{N}
$$

ist eine Linearkombination aus normierter Steuerkraft und Versorgungsniveau. Für sie gilt:

$$
\min \left\{v, \bar{K}_{i}\right\}<\bar{F}_{i}<\max \left\{\overline{\mathrm{K}}_{i}, v\right\}, \overline{\mathrm{K}}_{i} \neq v
$$

und somit

1) Zur Umlage vgl. Seiler [1980], S. 66 . 


$$
\mathrm{v}<\overline{\mathrm{F}}_{\mathrm{i}}<\overline{\mathrm{K}}_{\mathrm{i}}, \overline{\mathrm{K}}_{\mathrm{i}}>\mathrm{v}
$$

$(9.12)$

$$
\begin{aligned}
\overline{\mathrm{K}}_{\mathrm{i}}<\overline{\mathrm{F}}_{\mathrm{i}}<\mathrm{v}, & \overline{\mathrm{K}}_{\mathrm{i}}<\mathrm{v} \\
\overline{\mathrm{F}}_{\mathrm{i}}=\mathrm{v}, & \overline{\mathrm{K}}_{\mathrm{i}}=\mathrm{v} .
\end{aligned}
$$

Es besteht also ein gewisses $M a \beta$ an fiskalischer Ungleichheit, das umso höher ist, je kleiner a und umso geringer, je größer a gewählt wird. Denn wegen (9.11) wird in jeder Gemeinde ein Finanzierungsüberschuß bzw. ein Finanzierungsdefizit geringer, wenn a steigt und größer, wenn a fällt. Der Ausgleichssatz eignet sich also sehr gut als Instrumentvariable zur Regulierung fiskalischer Ungleichheit, und zwar unabhängig von der Höhe der Schlüsselmasse. 
$\underline{\text { SchluBbemerkungen }}$

Die vorliegende Untersuchung konnte natürlich bei weitem nicht alle Fragen behandeln, die das system der Schlüsselzuweisungen aufwirft. Für die weitere Forschung bleiben noch viele probleme ungelöst. Wir haben uns beispielsweise noch nicht mit der Frage befaßt, welche Nivellierungshebesätze man seitens des Staates zur Erfassung der kommunalen Steuerkraft anlegen sollte und wie sie sich auf die Verteilungsergebnisse auswirken. 1) Ein schwerwiegendes problem stellt nach wie vor auch die Messung des kommunalen Finanzbedarfs dar. Es ist z.B. noch völlig unklar, ob das System der Schlüsselzuweisungen einen ordinalen oder kardinalen Bedarfsindex verlangt und wie ein geeigneter Index aussehen müßte. Die zur zeit praktizierte Form der Bedarfsmessung ist ja keineswegs theoretisch fundiert und es ist durchaus denkbar, daß ein anderer Bedarfsindex, der prinzipiell die gleiche Plausibilität beanspruchen kann, eine ganz andere Verteilung erzeugt. ${ }^{2}$ )

Ein möglicher Ausweg aus diesem Dilemma wäre, einen Index auf deduktivem Wege auszuwählen. Man fordert dabei von Bedarfsindizes axiomatisch bestimmte Eigenschaften. Im günstigsten Fall gibt es nur einen Index, der die gewünschten Eigenschaften aufweist. Oft ist es jedoch so, daß es mehrere Indizes mit diesen Eigenschaften gibt, was nicht weiter problematisch ist, solange sich diese Indizes in thren Ergebnissen nicht widersprechen. Für den Fall, daß Indizes mit den gleichen Eigenschaften den Finanzbedarf der Kommunen unterschiedlich einschätzen, bleibt nur die Möglichkeit, neue oder zusätzliche Axiome zu fordern, und das ganze procedere wieder von vorne $z u$ beginnen. Wir wollen diesen Gedankengang hier nicht weiter vertiefen ${ }^{3)}$, sondern damit nur aufzeigen, mit welchen Problemen man sich

1) Siehe dazu zuletzt Grimme [1985].

2) Siehe Hanusch, Kuhn [1985].

3) Zur Theorie der Bedarfsmessung siehe ausführlich Hanusch, Kuhn [1984]. 
noch auseinandersetzen muß, bis man über ein rationales system von $\mathrm{Zuweisungen} \mathrm{verfügen} \mathrm{wird.}$

Ungeachtet der verbleibenden Forschungsdefizite können die in dieser Arbeit gewonnenen Erkenntnisse in der Praxis nutzbringend angewendet werden. So dürfte es nun für die Kommunalpolitiker ohne weiteres möglich sein, anhand relativ einfacher Kriterien zu überprüfen, welche Verteilung für ihre Kommune optimal wäre und wie gut sie bei der Verteilung, wie sie in einem bestimmten Jahr tatsächlich erfolgt, gestellt ist. Dadurch wird das ganze System für die Kommunen transparenter.

Von einer größeren Transparenz profitieren natürlich auch die Landespolitiker. Bei Reformen des Finanzausgleichs lassen sich die Folgen bestimmter Gesetzesänderungen, zumindest was die Ausschüttungsquote oder die Schlüsselmasse angeht, genau absehen. Man weiß auch, wie diese Variablen gehandhabt werden müssen, wenn fiskalische Ungleichheit global reduziert oder eine Gemeindegruppe gezielt begünstigt werden soll. Die Arbeit hat aber auch deutlich gemacht, daß man mit dem gegenwärtigen System schnell an Grenzen stößt, wenn man redistributive $\mathrm{Ziele}$ ernsthaft verfolgen will. Fiskalische Gleichheit wird man praktisch nie realisieren können, weshalb man den Politikern nur empfehlen kann, die Schlüsselzuweisungen langfristig durch ein einfacheres und effektiveres system, etwa in der Art, wie es hier vorgeschlagen wurde, zu ersetzen. Allerdings ließe es sich dabei kaum umgehen, reichere Gemeinden zugunsten ärmerer auch absolut schlechter zu stellen.

Die Politiker haben sich dazu jedoch noch nicht entschließen können. Es ist zu vermuten, daß sie die Intransparenz der gegenwärtigen Regelung noch nicht einmal als Nachteil empfinden, denn über ein system, bei dem vermeintlich niemand schlechter gestellt werden muß, ist im Prozeß der politischen Willensbildung natürlich eher ein Konsens zu erzielen. Das ist viel- 
leicht auch der Grund dafür, weshalb die Schlüsselzuweisungen in ihrer Grundstruktur nun schon so lange Bestand haben, obwohl ihre Schwächen wirklich nicht $z u$ übersehen sind. 
(1) Die Schlüsselzuweisungen als ein bedeutender Teil des kommunalen Finanzausgleichs werden nach einem regelgebundenen Verfahren auf die Kommunen verteilt. Danach erhält eine Kommune einen bestimmten Prozentsatz des Unterschieds zwischen ihrem Finanzbedarf und ihrer Steuerkraft als Schlüsselzuweisung. Kommunen, in denen die steuerkraft mindestens so hoch wie der Finanzbedarf ist, sind abundant.

(2) In jedem Jahr kann immer nur eine fest vorgegebene Summe, die sogenannte Schlüsselmasse, an die Kommunen verteilt werden. Sie wird im Grunde von den Steuereinnahmen eines Landes begrenzt. Um die verfügbare Schlüsselmasse genau auszuschöpfen, wird der Finanzbedarf der Kommunen endogenisiert. Dieser setzt sich multiplikativ aus der veredelten Einwohnerzahl, die als gemeindespezifische Bedarfskennziffer anzusehen ist, und dem Grundbetrag zusammen. Der Grundbetrag ist für alle Gemeinden gleich und wird endogen so bestimmt, daß sich ein Gleichgewicht zwischen der Schlüsselmasse und der Summe der $\mathrm{Z}$ uweisungen ergibt. Man kann ihn daher als durchschnittliches Versorgungsniveau interpretieren.

(3) Ordnet man die Gemeinden nach ihrer relativen Armut, dann gibt es $z u$ jedem Versorgungsniveau genau eine Gemeinde in dieser ordnung, die gerade noch Zuweisungen erhält. Alle reicheren Gemeinden sind abundant. Für die relative Armut einer Kommune ist dabei das Verhältnis aus ihrer Steuerkraft und ihrer Bedarfskennziffer maß-gebend. Mit dem Versorgungsniveau ändert sich gleichzeitig auch die Zahl der zuweisungsberechtigten Gemeinden. Sie wird größer, wenn das Versorgungsniveau zunimmt und kleiner, wenn es sinkt. 
(4) Bei der Bestimmung des Versorgungsniveaus ist eine Interdependenz zwischen zuweisungsberechtigten Gemeinden und dem Versorgungsniveau zu beachten, die sich in einer impliziten Gleichgewichtsbedingung ausdrückt. Man kann zeigen, daß eine Gleichgewichtslösung existiert und eindeutig ist. Daraus ergibt sich eine eindeutige Verteilung der Schlüsselzuweisungen. Die Statistischen Landesämter ermitteln sie mit einem numerischen Iterationsverfahren. $\mathrm{Zu}$ diesem Verfahren haben wir drei Alternativen vorgeschlagen, die noch etwas einfacher und schneller sein dürften.

(5) Was den Stand der Literatur angeht, so ist das System der Schlüsselzuweisungen mit formal-analytischen Methoden noch nicht untersucht worden. Das liegt daran, daß es wegen der vorhandenen Interdependenz zwischen Versorgungsniveau und zuweisungsberechtigten Gemeinden bisher noch nicht gelungen ist, das Versorgungsniveau analytisch $\mathrm{zu}$ bestimmen und von interessierenden Variablen funktional abhängig $z u$ machen. In dieser Arbeit konnte dieses Problem gelöst werden. Dadurch ist es erst möglich geworden, auf theoretischem Wege durch Variation von Variablen, wie der Schlüsselmasse oder der Ausschüttungsquote, beliebig viele alternative Verteilungen zu erzeugen, in denen die Schlüsselzuweisungen von den Variablen funktional abhängen. Ziel der Arbeit ist es gewesen, diese Verteilungen anhand geeigneter Kriterien danach $\mathrm{zu}$ beurteilen, inwieweit sie staatliche und kommunale ziele erfüllen.

(6) Der Staat verbindet mit Schlüsselzuwejsungen in erster Linie die Absicht, die fiskalische Ungleichheit der Kommunen zu reduzieren. Darüberhinaus ist aus staatlicher Sicht auch zu fragen, ob es überhaupt eine Verteilung gibt, mit der fiskalische Gleichheit unter den 
Kommunen realisiert werden kann. Eine Kommune beurteilt eine Verteilung in erster Linie danach, ob sie mit ihr ihre eigene Finanzausstattung, also ihre absolute Position, oder ihren Finanzanteil, also ihre relative Position unter den Gemeinden, verbessern kann. Für sie ist somit eine Verteilung optimal, die ihre Finanzausstattung bzw. ihren Finanzanteil maximiert.

(7) In der Analyse haben wir zunächst die Situation, die sich bei einer beliebigen Verteilung der Schlüsselzuweisungen ergibt, mit dem zustand verglichen, bei dem die Kommunen nur über ihre eigene steuerkraft verfügen können. Man kann zeigen, daß die Rangordnung der Armut mit Schlüsselzuweisungen nicht verändert werden kann, ganz gleich, welche Verteilung gewählt wird. Deshalb kommt es nie vor, daß eine Gemeinde mit Schlüsselzuweisungen reicher wird als die Gemeinden, die vorher schon reicher waren als sie.

(8) Die absolute Position der zuweisungsberechtigten Gemeinden verbessert sich, denn deren Finanzausstattung ist mit zuweisungen natürlich höher als vorher. Infolgedessen verringert sich auch ihr Finanzierungsdefizit, es kann aber nur mit Ausschüttungsquoten von 1 ganz zum Verschwinden gebracht werden. Die absolute Position der abundanten Gemeinden verändert sich nicht. Sie verfügen im Vergleich $\mathrm{zu}$ den ärmeren Kommunen aber nach wie vor über Finanzierungsüberschüsse, denn es gibt eine Besitzstandsgarantie: Keire Kommune muß von ihren Steuereinnahmen an ärmere Kommunen etwas abgeben.

(9) Betrachtet man die relativen Positionen der Kommunen, so gibt es wieder zu jeder Verteilung eine ganz bestimmte Kommune in der Rangordnung der Armut, die die Grenze angibt, bis zu der alle ärmeren Kommunen durch Zuweisungen relativ bessergestellt werden. Die reicheren 
Kommunen stellen sich entsprechend relativ schlechter. Bemerkenswert ist hier jedoch, daß nicht alle Gemeinden, die zuweisungsberechtigt sind, auch zu den bessergestellten gehören müssen. Bei den reicheren Gemeinden unter ihnen kann es durchaus sein, daß sich ihre relative Position sogar verschlechtert, obwohl sie selbst Zuweisungen erhalten.

(10) Was die fiskalische Ungleichheit der Kommunen bei einer beliebigen Verteilung angeht, so wird sie, verglichen mit der steuerkraftverteilung, zumindest nicht größer, meist reduziert sie sich sogar. Das ist wohl auch das mindeste, was der staat von den Schlüsselzuweisungen verlangen muB. Allerdings kann fiskalische Gleichheit nicht verwirklicht werden, wenn man nur Ausschüttungsquoten kleiner als 1 zuläßt. In diesem Fall würde fiskalische Gleichhelt die Gleichverteilung der auf einen repräsentativen Einwohner bezogenen steuerkraft implizieren. Das ist aus staatlicher sicht natürlich ein trauriges Resultat, denn es besagt auch, daß immer dann, wenn sich die Gemeinden in ihrer Armut unterscheiden, eine Verteilung, mit der sich fiskalische Gleichheit erzeugen ließe, nicht existiert. So weisen die zuweisungsberechtigten Gemeinden stets ein Finanzierungsdefizit auf, während in den reicheren Gemeinden in aller Regel ein Finanzierungsüberschuß vorhanden ist.

(11) Fiskalische Gleichheit ist überhaupt nur mit einer Ausschüttungsquote von $1 \mathrm{zu}$ erreichen. Dann können alle zuweisungsberechtigten Gemeinden ihren Finanzbedarf decken. Das reicht aber noch nicht aus, denn in den reichen Gemeinden verbleibt wegen der Besitzstandsgarantie nach wie vor ein Finanzierungsüberschuß. Fiskalische Gleichheit stellt sich erst ein, wenn man bei einer Ausschüttungsquote von 1 das Versorgungs- 
niveau so weit anhebt, bis keine Gemeinde mehr abundant ist. Dies ist allerdings nur als eine theoretische Möglichkeit anzusehen, wenn man bedenkt, daß das Versorgungsniveau von der Schlüsselmasse beschränkt wird und Schlüsselmassen in dem dafür benötigten Umfang, den man genau angeben kann, ganz unrealistisch sind.

(12) Fiskalische Ungleichheit wird man mit Schlüsselzuweisungen praktisch nie beseitigen können, so daß man versuchen muB, sie wenigstens zu reduzieren. Dafür kommen theoretisch zwei Variablen, die Schlüsselmasse und die Ausschüttungsquote, in Frage. Wenn man sich die Eigenschaft zunutze macht, da $\beta$ bei allen Alternativen die Rangordnung der Gemeinden nach der Armut immer erhalten bleibt und das Lorenzkurven-Kriterium für diesen Fall umformuliert, dann kann man analytisch zeigen, daß die fiskalische Ungleichheit mit steigender Schlüsselmasse abnimmt. Das gleiche gilt für die Ausschüttungsquote: Verteilungen mit höherer Ausschüttungsquote sind progressiver. Entsprechend nimmt natürlich die fiskalische Ungleichheit mit sinkender Schlüsselmasse und niedrigeren Ausschüttungsquoten $z u$.

(13) Die aufgezeigten Strategien, mit denen die fiskalische Ungleichheit theoretisch reduziert werden könnte, sind in der Realität nicht praktizierbar. Faktisch werden der Redistribution durch die Schlüsselmasse sehr enge Grenzen gesetzt. Man befindet sich hier in einem Dilemma, aus dem es innerhalb des Systems keinen Ausweg gibt. Einerseits ist das system so angelegt, daß fiskalische Ungleichheit in nennenswertem Umfang nicht unabhängig von der Schlüsselmasse reguliert werden kann, andererseits eignet sich diese Variable kaum, oder jedenfalls nur sehr bedingt, als Instrumentvariable. 
Die Abhängigkeit der fiskalischen Ungleichheit von der Schlüsselmasse ist somit als das Grundubel dieser Konstruktion anzusehen. Die systemimmanenten Restriktionen können nur überwunden werden, wenn man ein Umlageverfahren einführt, das die Finanzierungsüberschüsse der reichen Gemeinden abschöpft. Dazu schlagen wir ein besonders handliches system von zuweisungen vor, bei dem sich die fiskalische Ungleichheit unabhängig von der Schlüsselmasse mit der Ausschüttungsquote beliebig reguiieren läßt.

(14) Wie sich progressive und regressive Verteilungen im derzeitigen system auf Gemeindegruppen auswirken, kann man sehr genau sagen. Geht man von einer bestimmten, aber beliebigen Schlüsselmasse aus (status quo) und erhöht oder verringert diese Schlüsselmasse um einen bestimmten Betrag, dann lassen sich jeweils drei Gruppen von Gemeinden unterscheiden. Eine Gruppe von armen Kommunen besteht aus denjenigen, die vorher und nachher zuweisungen erhalten. Einer Gruppe mit reichen Gemeinden gehören diejenigen an, die weder vorher noch nachher Zuweisungen bekommen. Dazwischen gibt es eine Gruppe mit Gemeinden, die bei einer Erhöhung der Schlüsselmasse zum Kreis der zuweisungsberechtigten Gemeinden hinzukommen und bei einer Verringerung der Schlüsselmasse aus diesem Kreis ausscheiden.

(15) Was die Veränderung der absoluten Positionen der Gemeinden in den einzelnen Gruppen angeht, so fällt auf, daß der Anstieg der normierten Finanzausstattung be 1 den ärmsten Gemeinden am größten ist, und nicht, wie man vielleicht intuitiv erwarten könnte, bei den Gemeinden der mittleren Gruppe. Die armen Gemeinden haben bei einem Rückgang der Schlüsselmasse dafür aber auch die größten Einbußen zu verzeichnen. 
(16) Betrachtet man die Verschiebung der relativen Positionen, so gibt es wieder eine Gemeinde in der Rangordnung der Armut, die die Grenze angibt, bis zu der bei steigender Schlüsselmasse alle ärmeren Gemeinden einen steigenden Finanzanteil und alle reicheren Gemeinden einen sinkenden Finanzanteil aufweisen. Diese Gemeinde liegt entweder in der armen oder mittleren Gruppe. Das kommt ganz auf die Elastizität des Grundbetrags in Abhängigkeit von der Finanzmasse an. Ist sie größer als 1/a, dann liegt die Gemeinde in der mittleren Gruppe, sonst in der armen Gruppe.

(17) Analoge tberlegungen kann man bei einer Variation der Ausschüttungsquote anstellen. Man geht von der Ausschüttungsquote im status quo aus und erhöht oder verringert sie un einen bestimmten Faktor. Zunächst einmal ist festzuhalten, daß sich die Zahl der zuweisungsberechtigten Gemeinden erhöht, wenn die Ausschüttungsquote sinkt, und verringert, wenn die Ausschüttungsquote steigt. Allerdings kann diese $\mathrm{Zahl}$ nicht beliebig klein werden, weil eine bestimmte zahl von Gemeinden bei jeder Ausschüttungsquote zuweisungsberechtigt ist. Diese Zahl hängt nur noch von der Schlüsselmasse $a b$ und ist bei höheren Schlüsselmassen größer als bei niedrigeren.

Bei konstanter Schlüsselmasse ist jede Veränderung der Ausschüttungsquote mit einer Umverteilung der Schlüsselmasse verbunden. Man kann deshalb keine Gemeinde absolut besserstellen, ohne mindestens eine andere Gemeinde gleichzeitig absolut schlechter zu stellen. Die relative und absolute Position der Gemeinden aus der reichen Gruppe ändert sich nie. Die Umverteilung findet deshalb nur zwischen Gemeinden der armen und der mittleren Gruppe statt. Bei einer Erhöhung der Ausschüttungsquote verschlechtert sich die absolute und relative Position der Gemeinden aus der mittleren 
Gruppe, es können aber auch noch die reichsten aus der armen Gruppe betroffen sein. Eine Senkung des Ausgleichssatzes hat natürlich genau die umgekehrte wirkung. Es sind nun die ärmsten Gemeinden aus der armen Gruppe, die zugunsten der mittleren Gruppe, unter Umständen aber auch noch zugunsten der reichsten Gemeinden aus ihrer eigenen Gruppe schlechter gestellt werden.

(18) Kommen wir nun zu den optimalen Schlüsselmassen für die Kommunen. Für jede Kommune kann man eine Untergrenze für die Schlüsselmasse angeben, ab der die Kommune erst zuweisungsberechtigt wird. Diese Grenze ist umso größer, je reicher die Gemeinde ist. Ab dieser Grenze steigt die Zuweisung in jeder Kommune streng monoton mit der Schlüsselmasse, allerdings mit abnehmenden Zuwächsen, wenn Intervallgrenzen überschritten werden.

Infolgedessen ist immer die größtmögliche Schlüsselmasse optimal, wenn die Kommunen sich an ihrer absoluten Position orientieren und die Schlüsselmasse nach oben begrenzt ist. Die Gemeinden, die bei der Obergrenze noch nicht zuweisungsberechtigt sind, sind natürlich zwischen allen möglichen Schlüsselmassen indifferent. Orientieren sich die Kommunen an ihren relativen Positionen, so ist für sehr reiche Gemeinden eine Schlüsselmasse von $O$ und für sehr arme die maximal mögliche Schlüsselmasse optimal. Für die übrigen Gemeinden kann man keine generellen Aussagen machen. Es müssen für jede Gemeinde einzeln bestimmte Kriterien überprüft werden, aus denen sich jeweils die optimale Schlüsselmasse ergibt.

(19) Optimale Ausschüttungsquoten für die Gemeinden erhält man einfach durch Maximierung der Schlüsselzuweisungen, denn damit stellen sich die Gemeinden sowohl absolut, als auch relativ gesehen am besten. 
Hier kann man ganz allgemein zeigen, daB die optimale Ausschüttungsquote einer ärmeren Gemeinde nicht kleiner ist als die einer reicheren Gemeinde. Ordnet man also die optimalen Ausschüttungsquoten in aufsteigender Reihenfolge, so verhält sich diese ordnung genau umgekehrt wie die Ordnung der Gemeinden nach der Armut.

(20) Die optimalen Verteilungen der Gemeinden würden sich in der Regel also alle voneinander unterscheiden. In jedem Jahr kann immer nur eine Verteilung realisiert werden. Diese richtet sich auch nicht nach den Interessen einzelner Kommunen, sondern es ist diejenige Verteilung, die aus den gesetzlichen Bestimmungen resultiert und mit der der staat seine eigenen ziele verwirklichen möchte. Aus der Sicht einer Kommune weicht diese Verteilung natürlich mehr oder weniger von der Verteilung $a b$, die für sie optimal wäre. Welche Kommunen ihrem optimalzustand relativ nahe kommen und welche davon weit entfernt sind, kann man nicht allgemein sagen. Dazu müßte man in jedem einzelnen Fall die optimale Schlüsselmasse und Ausschüttungsquote mit der verfügbaren Schlüsselmasse und der gesetzlich festgelegten Ausschüttungsquote vergleichen. Die Interessen der armen Gemeinden und die des Staates gehen sicherlich in die gleiche Richtung, denn beide präferieren hohe Schlüsselmassen und hohe Ausgleichssätze. In der Realität dürften die armen Gemeinden allerdings immer noch stark benachteiligt sein, weil die tatsächlichen Schlüsselmassen und Ausgleichssätze zur zeit wesentlich niedriger sind, als sie sich staat und arme Gemeinden eigentlich wünschen. 
VERZEICHNIS DER WICHTIGSTEN SYMBOLE

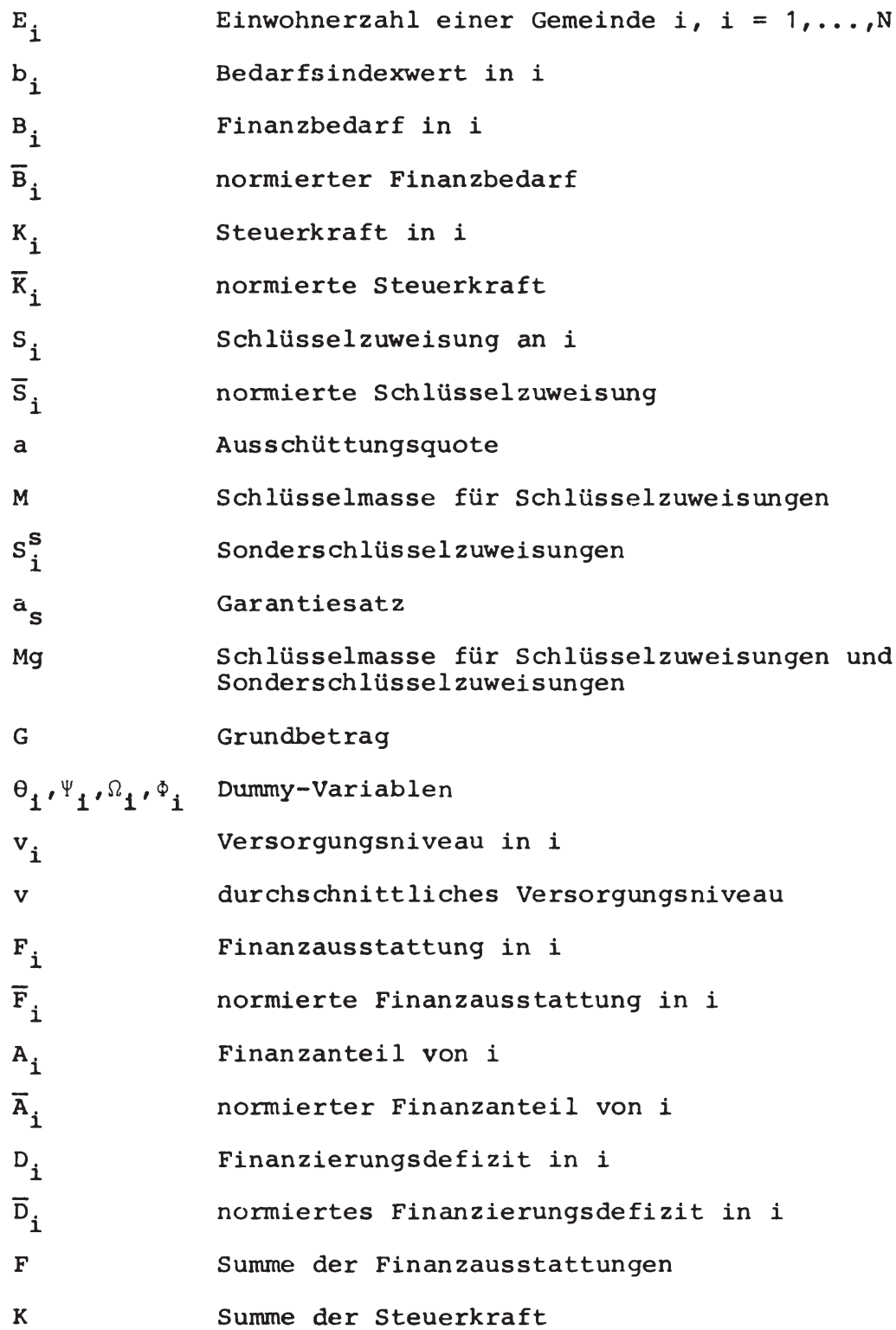


A

s

L

$\mathrm{L}_{\mathrm{P}}$

$\mathrm{L}_{\mathrm{I}}$

$\Delta \mathrm{M}$

$\lambda$

$\Delta \mathbf{S}_{\mathbf{i}}$

$\Delta F_{i}$

$\mathrm{M}^{*}$

*

$a_{1}$

$\mathrm{Z}_{\text {i }}$

${ }^{\varepsilon} G, F$

Menge aller alternativen Verteilungen

Verteilung der Schlüsselzuweisungen

Lorenzpräferenz

Lorenzdominanz

Lorenzindifferenz

Anderung der Schlüsselmasse

Anderung der Ausschüttungsquote

Anderung der Schlüsselzuweisung in Abhängigkeit von $\lambda$ oder $\Delta M$

Anderung der Finanzausstattung

optimale Schlüsselmasse für i

optimale Ausschüttungsquote für i

Zuweisung an $i$

Elastizität von $G$ nach $F$ 


\section{VERZE ICHNIS DER GESETZE}

(Stand 1.1.1985)

\section{$\underline{\text { PreuBen }}$}

Preußisches Finanzausgleichsgesetz vom 10. November 1938. Preußische Gesetzsammlung 1980, Nr. 22, S. 108-118.

\section{Baden-Württemberg}

Gesetz über den kommunalen Finanzausgleich (FAG) in der Fassung vom 23. Januar 1984 (GBl. S. 129).

\section{Bayern}

Gesetz über den Finanzausgleich zwischen Staat, Gemeinden und Gemeindeverbänden (Finanzausgleichsgesetz - FAG) vom 31. Januar 1984 (GVBl. S. 5).

\section{Hessen}

Gesetz zur Regelung des Finanzausgleichs (Finanzausgleichsgesetz - FAG -) in der Fassung vom 29. Juni 1984 (GVB1. I 194-206).

\section{$\underline{\text { Niedersachsen }}$}

Gesetz über den Finanzausgleich (FAG) in der Fassung vom 11. November 1981. (Nieders. GVBI. S. 339), geändert durch Artikel I und II des Gesetzes vom 4. Oktober 1983 (Nieders. GVB1. S. 253).

\section{Nordrhein-Westfalen}

Gesetz zur Regelung des Finanz- und Lastenausgleichs mit den Gemeinden und Gemeindeverbänden für das Haushaltsjahr 1980 (Finanzausgleichsgesetz 1980 - FAG 1980) vom 4. März 1980 (GVBl. Nr. 12, S. 122-130). 


\section{Rheinland-Pfalz}

Landesgesetz über den Finanzausgleich in Rheinland-Pfalz (Finanzausgleichsgesetz - FAG -) vom 28. Oktober 1977 (GVBl. S. 353) - geändert durch Artikel 2 d. LG vom 22.3.1978 (GVBl. S. 143), Artikel 3 d. LG vom 2.7.1980 (GVBl. S. 146), Artikel 5 d. LG vom 18.12.1981 (GVBl. S. 331), Artikel 2 d. LG vom 8.2.1982 (GVBl. S. 65), § 22 d. LG vom 3.12.1982 (GVBl. S. 431), § 48 d. LG vom 22.12.1982 (GVB1. S. 463) und Landesgesetz zur Änderung des Finanzausgleichsgesetzes vom 6. April 1984 (GVBl. S. 77).

\section{Saarland}

Kommunalfinanzausgleichsgesetz - KFAG - vom 12. Juli 1983

( $\mathrm{ABl}$. S. 462-470) - zuletzt geändert 19.10.1983 (ABI. S. 717).

\section{Schleswig-Holstein}

Gesetz über den Finanzausgleich in Schleswig-Holstein (Finanzausgleichsgesetz - FAG) in der Fassung vom 16. Januar 1984 (GVBl. S. 26) - zuletzt geändert 29.11.1984 (GVBI. S. 246). 


\section{LITERATURVERZE ICHNIS}

Bohley, E., Foohs, L.: Handbuch des gemeindlichen Steverrechts, Teil IV/Finanzausgleich. München $1984^{6}$.

Bös, D.: Eine ökonomische Theorie des Finanzausgleichs. Wien - New York 1971.

Brecht, A.: Internationaler Vergleich der öffentlichen Ausgaben. Berlin - Leipzig 1932.

Deubel, I.: Mängel im Finanzausgleichssystem des Landes Nordrhein-Westfalen. In: W. Hoppe (Hrsg.): Reform des kommunalen Finanzausgleichs. Köln 1985, S. 61 - 86.

Gläser, M.: Die staatlichen Finanzzuweisungen an die Gemeinden - Gestaltungskriterien, Effektivität, Reform. Frankfurt 1981 .

Grimme, L.: Neuberechnung der Steuerkraft im kommunalen Finanzausgleich - dargestellt am Beispiel des Landes Bayern. In: Akademie für Raumforschung und Landesplanung (Hrsg.): Räumliche Aspekte des kommunalen Finanzausgleichs. Fus Bd. 159, Hannover 1985, S. 159-200.

Hansmeyer, K.H.: Der kommunale Finanzausgleich als Instrument zur Förderung zentraler Orte. In: D. Pohmer (Hrsg.): Probleme des Finanzausgleichs II. Schriften des Vereins für Socialpolitik NF 96/II, Berlin 1980, S. $83-150$.

Hansmeyer, K.H., Kops, M.: Die Popitz'sche Hauptansatzstaffel. In: Akademie für Raumforschung und Landesplanung (Hrsg.): Räumliche Aspekte des Finanzausgleichs. Fus 159, Hannover 1985, S. 15-54. 
Hanusch, H., Kuhn, T.: Theorie des kommunalen Finanzbedarfs. Augsburg 1984 (unveröffentlicht).

Hanusch, H., Kuhn, T.: Messung des kommunalen Finanzbedarfs - Ein alternativer Ansatz für die Schlüsselzuweisungen. In: Akademie für Raumforschung und Landesplanung (Hrsg.): Räumliche Aspekte des Finanzausgleichs. Fus 159, Hannover 1985, S. 55-74.

Hoppe, W.: Die Leistungen der Länder an die Gemeinden und Kreise im kommunalen Finanzausgleich. In: W. Hoppe (Hrsg.): Reform des kommunalen Finanzausgleichs. Köln 1985 , S. $87-102$.

Karrenberg, H., Münstermann, E.: Gemeindefinanzbericht 1983,85. Der Städtetag 2(1983), S. 63-108, 2(1985), S. 72-110.

Kops, M.: Die regionalen Verteilungswirkungen des kommunalen Finanzausgleichs. Frankfurt 1983.

Leibfritz, W., Teschner, S.: Der EinfluB des Steuersystems und des kommunalen Finanzsystems auf die Landesentwicklung. Schriftenreihe des IFO-Instituts für Wirtschaftsforschung Nr. 108, Berlin 1981.

Münstermann, E.: Die Berücksichtigung zentralörtlicher Funktionen im kommunalen. Finanzausgleich. Opladen 1975.

Münstermann, E., Becker, H.: Finanzausgleichsleistungen an Kommunen. DST-Beiträge zur Finanzpolitik, H. 7, Köln 1978.

Musgrave, R.A.: Approaches to a Fiscal Theory of Political Federalism. In: National Bureau of Economic Research (ed.): Public Finances: Needs, Sources and Utilization. Princeton 1961, s. 97-133. 
Neumark, F.: Bemerkungen zu einigen ökonomischen Aspekten der grundgesetzlichen Vorschriften über die Einheitlichkeit der Lebensverhältnisse in der Bundesrepublik Deutschland. In: W. DreiBig (Hrsg.): Probleme des Finanzausgleichs I. Berlin 1978.

Pollak, H., VoB, W.: Die Berücksichtigung von Spillover-Effekten im kommunalen Finanzausgleich. In: Akademie für Raumforschung und Landesplanung (Hrsg.): Räumliche Aspekte des Finanzausgleichs. Fus 159, Hannover 1985, S. 133-158.

Popitz, J.: Der künftige Finanzausgleich zwischen Reich, Ländern und Gemeinden. Berlin 1932

Postlep, R.D.: Räumliche Effekte der Steuerkraft- und Finanzbedarfsermittlung bei den Schlüsselzuweisungen. In: Akademie für Raumforschung und Landesplanung (Hrsg.): Räumliche Aspekte des kommunalen Finanzausgleichs. Fus 159, Hannover 1985, S. 201-234.

Seiler, G.: Ziele und Mittel des kommunalen Finanzausgleichs - Ein Rahmenkonzept für einen aufgabenbezogenen kommunalen Finanzausgleich. In: D. Pohmer (Hrsg.): Probleme des Finanzausgleichs II. Schriften des Vereins für Socialpolitik NF 96/II, Berlin 1980, S. $11-82$.

Smekal, C.: Transfers zwischen Gebietskörperschaften (TG) Ziele und Ausgestaltungsprobleme. In: D. Pohmer (Hrsg.): Probleme des Finanzausgleichs II. Schriften des Vereins für Socialpolitik NF 96/II, Berlin 1980, S. 151-220.

Stehling, F.: Wirtschaftliche Kennzahlen. Karlsruhe 1983 (unveröffentlicht).

Thiem, H.: Kommunale Finanzwirtschaft. Köln 1984. 
Weber, M.: Die Schlüsselzuweisungen. Analyse eines Instruments des kommunalen Finanzausgleichs. Frankfurt 1981.

Zimmermann, H., Henke, K.D.: Einführung in die Finanzw1ssenschaft. München $1982^{3}$.

Zimmermann, H.: Allgemeine Probleme und Methoden des Finanzausgleichs. In: Handbuch der Finanzwissenschaft Bd. IV. Tübingen $1983^{3}$, S. 3-53. 
Thomas Kuhn - 978-3-631-75193-0

Downloaded from PubFactory at 01/11/2019 07:16:43AM

via free access 


\section{FINANZWISSENSCHAFTLICHE SCHRIFTEN}

Band 1 Werner Steden: Finanzpolitik und Einkommensverteilung. Ein Wachstums- und Konjunkturmodell der Bundesrepublik Deutschland. 1979.

Band 2 Rainer Hagemann: Kommunale Finanzplanung im föderativen Staat. 1976.

Band 3 Klaus Scherer: Maßstăbe zur Beurteilung von konjunkturellen Wirkungen des offentlichen Haushalts. 1977.

Band 4 Brita Steinbach: "Formula Flexibility" - Kritische Analyse und Vergleich mit diskretionărer Konjunkturpolitik. 1977.

Band 5 Hans-Georg Petersen: Personelle Einkommensbesteuerung und Inflation. Eine theoretisch-empirische Analyse der Lohn- und veranlagten Einkommensteuer in der Bundesrepublik Deutschland. 1977.

Band 6 Friedemann Tetsch: Raumwirkungen des Finanzsystems der Bundesrepublik Deutschland. Eine Untersuchung der Auswirkungen der Finanzreform von 1969 auf die Einnahmenposition der untergeordneten Gebietskorperschaften und ihrer regionalpolitischen Zieladåquanz. 1978.

Band 7 Wilhelm Prahler: Normative Theorie der fiskalischen Besteuerung. Ein methodologischer und theoretischer Beitrag zur Integration der normativen Besteuenungstheorie in der Wohlfahrtstheorie. 1978.

Band 8 Wolfgang Wiegard: Optimale Schattenpreise und Produktionsprogramme für offentliche Unternehmen. Second-Best Modelle im finanzwirtschaftlichen Staatsbereich. 1978.

Band 9 Hans P. Fischer: Die Finanzienung des Umweltschutzes im Rahmen einer rationalen Umweltpolitik. 1978.

Band 10 Rainer Paulenz: Der Einsatz finanzpolitischer Instrumente in der Forschungs- und Entwicklungspolitik. 1978.

Band 11 Hans-Joachim Hauser: Verteilungswirkungen der Staatsverschuldung. Eine kreislauftheoretische Inzidenzbetrachtung. 1979.

Band 12 Gunnar Schwarting: Kommunale Investitionen. Theoretische und empirische Untersuchungen der Bestimmungsgründe kommunaler Investitionstătigkeit in NordrheinWestfalen 1965-1972. 1979.

Band 13 Hans-Joachim Conrad: Stadt-Umland-Wanderung und Finanzwirtschaft der Kernstadte. Amerikanische Erfahnungen, grundsatzliche Zusammenhănge und eine Fallstudie tür das Ballungsgebiet Frankfurt am Main. 1980.

Band 14 Cay Folkers: Vermögensverteilung und staatliche Aktivităt. Zur Theorie distributiver Prozesse im Interventionsstaat. 1981.

Band 15 Helmut Fischer: US-amerikanische Exportiörderung durch die DISC-Gesetzgebung. 1981.

Band 16 Günter Ott: Einkommensumverteilungen in der gesetzlichen Krankenversicherung. Eine quantitative Analyse. 1981.

Band 17 Johann Hermann von Oehsen: Optimale Besteuerung. (Optimal Taxation). 1982.

Band 18 Richard Kossler: Sozialversicherungsprinzip und Staatszuschũsse in der gesetzlichen Rentenversicherung. 1982.

Band 19 Hinrich Steffen: Zum Handlungs- und Entscheidungsspielraum der kommunalen Investitionspolitik in der Bundesrepublik Deutschland. 1983.

Band 20 Manfred Scheuer: Wirkungen einer Auslandsverschuldung des Staates bei flexiblen Wechselkursen. 1983.

Band 21 Christian Schiller: Staatsausgaben und crowding-out-Effekte. Zur Effizienz einer Finanzpolitik keynesianischer Provenienz. 1983. 
Band 22 Hannelore Weck: Schattenwirtschaft: Eine Möglichkeit zur Einschrânkung der offentlichen Verwaltung? Eine Okonomische Analyse. 1983.

Band 23 Wolfgang Schmitt: Steuern als Mittel der Einkommenspolitik. Eine Ergânzung der Stabilitătspolitik? 1984.

Band 24 Wolfgang Laux: Erhơhung staatswirtschaftlicher Effizienz durch budgetăre Selbstbeschrănkung? Zur Idee einer verfassungsmäßig verankerten Ausgabengrenze. 1984.

Band 25 Brita Steinbach-van der Veen: Steuerinzidenz. Methodologische Grundlagen und empirisch-statistische Probleme von Lânderstudien. 1985.

Band 26 Albert Peters: Ókonomische Kriterien für eine Autgabenverteilung in der Marktwirtschaft. Eine deskriptive und normative Betrachtung für den Allokationsbereich. 1985.

Band 27 Achim Zeidler: Möglichkeiten zur Fortsetzung der Gemeindefinanzreform. Eine theoretische und empirische Analyse. 1985.

Band 28 Peter Bartsch: Zur Theorie der lăngerfristigen Wirkungen 'expansiver' Fiskalpolitik. Eine dynamische Analyse unter besonderer Berücksichtigung der staatlichen Budgetbeschrănkung und ausgewăhtter Möglichkeiten der offentlichen Defizittinanzierung. 1986.

Band 29 Konrad Beiwinkel: Wehrgerechtigkeit als finanzpolitisches Verteilungsproblem. Möglichkeiten einer Kompensation von Wehrungerechtigkeit durch monetare Transfers. 1986.

Band 30 Wolfgang Kitterer: Effizienz- und Verteilungswirkungen des Steuersystems. 1986.

Band 31 Heinz Dieter Hessler: Theorie und Politik der Personalsteuern. Eine Kritik ihrer Einkommens- und Vermogensbegriffe. 1987.

Band 32 Wolfgang Schert: Die beschaftigungspolitische und fiskalische Problematik der Arbeitgeberbeltrăge zur Rentenversicherung. Eine Auseinandersetzung mit der Kritik an der lohnbezogenen Beitragsbemessung. 1987.

Band 33 Andreas Mastle: Die Steuenunion. Probleme der Harmonisierung spezifischer Gütersteuern. 1987.

Band 34 Günter Ott: Internationale Verteilungswirkungen im Finanzausgleich der Europăischen Gemeinschaften. 1987.

Band 35 Heinz Haller: Zur Frage der zweckmaBigen Gestalt gemeindlicher Steuern. Ein Diskussionsbeitrag zur Gemeindesteuerreform. 1987.

Band 36 Thomas Kuhn: Schlusselzuweisungen und fiskalische Ungleichheit. Eine theoretische Analyse der Verteilung von Schlusselzuweisungen an Kommunen. 1988. 